\title{
Dynamics of a Predator-Prey Population in the Presence of Resource Subsidy under the Influence of Nonlinear Prey Refuge and Fear Effect
}

\author{
Sudeshna Mondal $\left(\mathbb{D},{ }^{1}\right.$ G. P. Samanta $\left(\mathbb{D},{ }^{1}\right.$ and Juan J. Nieto ${ }^{2}{ }^{2}$ \\ ${ }^{1}$ Department of Mathematics, Indian Institute of Engineering Science and Technology, Shibpur, Howrah 711103, India \\ ${ }^{2}$ Instituto de Matemáticas, Universidade de Santiago de Compostela, Santiago de Compostela 15782, Spain \\ Correspondence should be addressed to G. P. Samanta; gpsamanta@math.iiests.ac.in
}

Received 6 March 2021; Revised 7 May 2021; Accepted 11 June 2021; Published 5 July 2021

Academic Editor: Eberhard O. Voit

Copyright (c) 2021 Sudeshna Mondal et al. This is an open access article distributed under the Creative Commons Attribution License, which permits unrestricted use, distribution, and reproduction in any medium, provided the original work is properly cited.

In this work, our aim is to investigate the impact of a non-Kolmogorov predator-prey-subsidy model incorporating nonlinear prey refuge and the effect of fear with Holling type II functional response. The model arises from the study of a biological system involving arctic foxes (predator), lemmings (prey), and seal carcasses (subsidy). The positivity and asymptotically uniform boundedness of the solutions of the system have been derived. Analytically, we have studied the criteria for the feasibility and stability of different equilibrium points. In addition, we have derived sufficient conditions for the existence of local bifurcations of codimension 1 (transcritical and Hopf bifurcation). It is also observed that there is some time lag between the time of perceiving predator signals through vocal cues and the reduction of prey's birth rate. So, we have analyzed the dynamical behaviour of the delayed predator-prey-subsidy model. Numerical computations have been performed using MATLAB to validate all the analytical findings. Numerically, it has been observed that the predator, prey, and subsidy can always exist at a nonzero subsidy input rate. But, at a high subsidy input rate, the prey population cannot persist and the predator population has a huge growth due to the availability of food sources.

\section{Introduction}

In the ecological system, the predator-prey interaction is one of the most significant tools which is comparatively easy to observe in the field. But fear of the predator felt by the prey (indirect effect) also plays a vital role since its effect is stronger than direct predation $[1,2]$. The cost of fear can reduce the reproduction rate of prey because it affects the physiological condition of prey population. As a result, the prey species may get a long-term loss. In support of this, it is mentioned that, in the Greater Yellowstone Ecosystem, wolves (Canis lupus) affect the reproductive physiology of elk (Cervus elaphus) [3]. When the prey species recognize the predator signal (chemical/vocal), they spend more time to become keenly watchful to detect danger rather than in foraging. So, the birth rate of the scared prey reduces and adopts some survival mechanisms like starvation $[1,2]$. For examples, some birds react to the sound of predator with antipredator defenses $[1,2]$ and they flee from their nests at the first sign of danger [2]. This antipredator behaviour may affect survival and reproduction of the birds [2]. It has been experimentally investigated that, in the absence of direct killing, the reproduction of the offspring of song sparrows (Melospiza melodia) could be reduced by $40 \%$ as a result of impact of feeling fear created by the predator [4]. So, this reduction caused by the antipredator behaviour affects the birth rate and survival of offspring. Thus, the cost of fear (apart from direct predation) should be introduced in a predator-prey interaction. Mathematical formulation of the impact of fear on the two species prey-predator system has been initiated by Wang et al. [5] in 2016 introducing fear factor: $f(k, y)=(1 /(1+k y))$. It involves a parameter $k$ 
denoting the level of fear to represent the antipredator behaviour of the prey. Some research works have already been done on the ecological system under the influence of predation fear felt by prey species [6-17]. Moreover, the impact of fear in a two-species predator-prey model with prey refuge was analyzed by many researchers $[11,18,19]$.

In evolutionary biology, prey refuge is a concept which helps an organism to protect themselves from predation by hiding in an area inaccessible to the predator, for example, in a wolf-ungulate system, ungulates may seek refuge by migrating to areas outside the core territories of wolves. Also, it has many significant roles on the dynamics of predator-prey interactions: prey refuge may decrease the chance of extinction of prey. Researchers have mainly used the dynamic nature of predator-prey model with linear prey refuge (that is, $m x$ amounts of prey are unavailable to the predator, where $m \in(0,1)$ is the coefficient of refuge and $x$ is the biomass of prey species) with Volterra response [20-22]. Recently, Mondal and Samanta have studied the dynamics of the predator-prey system with prey refuge dependent on both species (that is, $m x y$ amounts of prey are free from the predator risk, where $0<1-m y<1, m$ is prey refuge coefficient, $x$ is the biomass of prey population, and $y$ is the biomass of predator population) in the presence of additional food (for details, see [23]). In 2020, Mondal and Samanta [11] have also analyzed the dynamics of predatorprey interaction having nonlinear prey refuge function $\Phi(x, y)=(m x y /(a+y))$ which is the amount of prey that are free from predation, where $a$ is half saturation constant and $y$ is the biomass of predator population.

Many experimental studies suspect that the introduction of resource subsidies may disrupt otherwise stable food web linkages [24-26]. Such concept is significant for resource management purposes. It is learnt that reintroduced wolves in Yellowstone Park switch to bison when their preferred ungulate prey, namely, elk, are rare in the concerned ecosystem [27]. Mathematically, the influence of resource subsidy on the predator-prey model has been initiated in the work of Nevai and Van Gorder [28]. They have discussed how different subsidy input rate may affect the prey and predator population to persist in the ecosystem.

Generalist predator can consume more than one food source: either multiple prey population or a combination of prey population and resource subsidy. There are many rich theoretical research on ecological systems involving generalist predator [29-31]. Also, there are a variety of real-life applications for such systems [32-34]. From literature surveys, it has been shown that generalist predator can persist in an ecosystem even if one particular prey species is going towards extinction [29-31].

In 2012, Nevai and Van Gorder [28] first extended the Kolmogorov model to a non-Kolmogorov predator-preysubsidy model. It has been observed that the predator-preysubsidy model occurs in the arctic foxes (predator), lemmings (prey), and seal carcasses (subsidy). Motivated by the works of Das and Samanta [35], Nevai and Van Gorder [28], and Xu et al. [36], we have analyzed the dynamical behaviour of a mathematical model of non-Kolmogorov form that includes the three components (predator, prey, and subsidy) with the impacts of nonlinear prey refuge function and the fear effect felt by the prey in the presence of the predator. To the best of our knowledge, there does not exist any mathematical model to explore the impact of fear effect incorporating nonlinear prey refuge function in predator-prey-subsidy interaction.

The organization of this work is structured as follows: in Section 2, a mathematical model has been formulated with the influences of nonlinear prey refuge and fear effect. Section 3 shows that the proposed model is well-behaved. In Section 4, feasibility criteria and stability of all the equilibria of the proposed system (in absence of delay) have been studied. The equilibria can change their stability nature through transcritical and Hopf bifurcation which are also analyzed in this section. Generally, the reduction of prey's birth rate due to the effect of fear will not be an instantaneous biological process but deviated through some time lag, so the study of time-delay $\tau$ is very meaningful to obtain the more realistic dynamics. So, Section 5 deals with the dynamic behaviour of the delayed system for two equilibrium points $E_{3}$ (subsidy free) and $E^{*}$ (interior), respectively. Section 6 provides the numerical computations which support the analytical calculations. Section 7 provides a brief conclusion about the system dynamics.

\section{Model Formulation}

In 2020, Mondal and Samanta [11] analyzed the dynamics of a delayed predator-prey interaction incorporating nonlinear prey refuge function under the influence of fear effect and additional food. Motivated by the work of Mondal and Samanta [11], we have first considered a predator-preysubsidy model with nonlinear prey refuge function where the prey and subsidy occur in the same habitat and they are both consumed by a single generalist predator according to the following differential equations:

$$
\begin{aligned}
& \frac{\mathrm{d} x}{\mathrm{~d} t}=r x-d_{1} x-a_{1} x^{2}-\frac{a_{2}(1-(m y /(a+y))) x y}{b_{1}+w+(1-(m y /(a+y))) x}, \quad x(0)>0, \\
& \frac{\mathrm{d} w}{\mathrm{~d} t}=A-d_{2} w-\frac{a_{3} w y}{b_{1}+w+(1-(m y /(a+y))) x}, \quad w(0)>0, \\
& \frac{\mathrm{d} y}{\mathrm{~d} t}=\frac{c_{1} a_{2}(1-(m y /(a+y))) x y}{b_{1}+w+(1-(m y /(a+y))) x}+\frac{c_{2} a_{3} w y}{b_{1}+w+(1-(m y /(a+y))) x}-d_{3} y, \quad y(0)>0,
\end{aligned}
$$


where $x$ is prey population, $w$ denotes the population of the subsidy, and $y$ is the generalist predator which exploits both the prey and subsidy. For example, wolves (predator) consume both deer (prey) and salmon carcasses (subsidy) [37].

The term $(1-(m y /(a+y))) x$ represents the quantity of prey available to the predator, i.e., $(m x y /(a+y))$ amounts of prey are free from predation risk where $(m x y /(a+y))$ is designated as nonlinear prey refuge function. Also, we have modeled the dynamics of a generalist predator with Holling type II [38-41] response function in the presence of nonlinear prey refuge function.

All parameters are positive (except $A \geq 0$ ) and biologically meaningful. Parameters are described in Table 1.

Apart from direct consumption, feeling of fear among the individuals of the prey species in presence of predator is very common in predator-prey interaction which changes life-history, behavioural responses, and reproduction capability of prey species. In ecology, effect of fear is a common factor, but there does not exist any considerable attention to introduce the impact of fear in the mathematical modeling.
Experimental studies indicate that the feeling of fear among the individuals of the prey species in presence of predator reduces the prey's birth rate. So, birth rate of prey species $r$ is multiplied by a monotone decreasing function $f(k, y)=(1 /(1+k y))$, where $k(\geq 0)$ is a level of fear [5]. The fear function $f(k, y)$ satisfies the following conditions:

(1) $f(0, y)=1$ : when there is no fear effect on the prey species, the birth rate of the prey is not reduced

(2) $f(k, 0)=1$ : when there is no predator, the birth rate of the prey species is not reduced in the presence of fear effect

(3) $(\partial f(k, y) / \partial k)<0$ : when fear effect increases, the birth rate of the prey reduces

(4) $(\partial f(k, y) / \partial y)<0$ : when predator species increases, prey population reduces

Our main focus is to analyze the dynamic nature of the predator-prey-subsidy model with the influence of nonlinear prey refuge and fear effect. So, system (1) can be modified in the following aspects:

$$
\begin{aligned}
& \frac{\mathrm{d} x}{\mathrm{~d} t}=\frac{r x}{1+k y}-d_{1} x-a_{1} x^{2}-\frac{a_{2}(1-(m y /(a+y))) x y}{b_{1}+w+(1-(m y /(a+y))) x}, \\
& \frac{\mathrm{d} w}{\mathrm{~d} t}=A-d_{2} w-\frac{a_{3} w y}{b_{1}+w+(1-(m y /(a+y))) x}, \\
& \frac{\mathrm{d} y}{\mathrm{~d} t}=\frac{c_{1} a_{2}(1-(m y /(a+y))) x y}{b_{1}+w+(1-(m y /(a+y))) x}+\frac{c_{2} a_{3} w y}{b_{1}+w+(1-(m y /(a+y))) x}-d_{3} y,
\end{aligned}
$$

with initial conditions:

$$
\begin{aligned}
& x(0)>0, \\
& w(0)>0, \\
& y(0)>0 .
\end{aligned}
$$

Throughout the analysis of this work, we have taken $c_{1}>c_{2}$ which is biologically meaningful.

\section{Positivity and Uniform Boundedness}

Theorem 1. Every solution of system (2) with (3) uniquely exists and is positive for all $t \geq 0$.

Proof. Solution $(x(t), w(t), y(t))$ of (2) with (3) exists and is unique on $[0, \xi)$, where $(0<\xi \leq+\infty)$ [42].

From (2) with (3),

$$
\begin{aligned}
& x(t)=x(0) \exp \left[\int_{0}^{t}\left\{\frac{r}{1+k y(\theta)}-d_{1}-a_{1} x(\theta)-\frac{a_{2}(1-(m y(\theta) /(a+y(\theta)))) y(\theta)}{b_{1}+w(\theta)+(1-(m y(\theta) /(a+y(\theta)))) x(\theta)}\right\} \mathrm{d} \theta\right]>0 \\
& y(t)=y(0) \exp \left[\int_{0}^{t}\left\{\frac{c_{1} a_{2}(1-(m y(\theta) /(a+y(\theta)))) x(\theta)}{b_{1}+w(\theta)+(1-(m y(\theta) /(a+y(\theta)))) x(\theta)}+\frac{c_{2} a_{3} w(\theta)}{b_{1}+w(\theta)+(1-(m y(\theta) /(a+y(\theta)))) x(\theta)}-d_{3}\right\} \mathrm{d} \theta\right]>0 .
\end{aligned}
$$


TABLE 1: Description of biologically meaningful parameters.

\begin{tabular}{lc}
\hline Parameters & Descriptions \\
\hline$r$ & Birth rate of the prey \\
$d_{1}$ & Natural death rate of the prey \\
$d_{2}$ & The subsidy decay rate \\
$a_{1}$ & Mortality rate due to intraspecific competition among the individuals of the prey population \\
$a_{2}$ & Consumption rate of the predator \\
$a_{3}$ & Maximum rate at which the predator consumes the subsidy \\
$b_{1}$ & Handling time assumed to be uniform over all food sources \\
$c_{1}$ & Conversion rate of the energy that the predator obtains from the target prey \\
$c_{2}$ & Conversion rate of the energy that the predator obtains from the subsidy \\
$A$ & Subsidy input rate \\
$m$ & Coefficient of prey refuge $(m \in(0,1))$ \\
$d_{3}$ & Mortality rate of the predator \\
$a$ & Half saturation constant for refuge function
\end{tabular}

Now, we claim that $w(t)>0$ for all $t \in[0, \xi)$. If it does not hold then there exists $t_{1} \in[0, \xi)$ such that $w\left(t_{1}\right)=0$, $\dot{w}(t) \leq 0$, and $w(t)>0$ on $\left[0, t_{1}\right)$. From the second equation of (2),

$$
\begin{aligned}
w\left(t_{1}\right)= & w(0) \exp \left[-\int_{0}^{t_{1}}\left(d_{2}+\frac{a_{3} y(\theta)}{b_{1}+w(\theta)+(1-(m y(\theta) /(a+y(\theta))) x(\theta)}\right) \mathrm{d}(\theta)\right] \\
& +\int_{0}^{t_{1}} A\left[\exp \left(\int_{t_{1}}^{u}\left(d_{2}+\frac{a_{3} y(\theta)}{b_{1}+w(\theta)+(1-(m y(\theta) /(a+y(\theta)))) x(\theta)}\right) \mathrm{d}(\theta)\right)\right] \mathrm{d} u>0,
\end{aligned}
$$

a contradiction with $w\left(t_{1}\right)=0$. So, $w(t)>0, \forall t \in[0, \xi)$.

Hence, solutions of (2) stay positive for all $t \geq 0$.
Theorem 2. All solutions of system (2) which start in $\mathbb{R}_{+}^{3}$ are asymptotically uniformly bounded.

Proof. Case 1: if $r>d_{1}$, from the first equation of (2),

$$
\begin{aligned}
\frac{\mathrm{d} x}{\mathrm{~d} t} & \leq \frac{r x}{1+k y}-d_{1} x-a_{1} x^{2} \\
& \leq r x-d_{1} x-a_{1} x^{2}\left[\text { since } \frac{r x}{1+k y} \leq r x\right] \\
& =\left(r-d_{1}\right) x\left(1-\frac{x}{\left(r-d_{1}\right) / a_{1}}\right), \\
\therefore \limsup _{t \rightarrow+\infty} x(t) & \leq \frac{r-d_{1}}{a_{1}}, \quad \text { since } r>d_{1} .
\end{aligned}
$$

Let us take $P=x+w+\left(y / c_{1}\right)$.

Differentiating both sides with respect to $t$, we obtain 


$$
\begin{aligned}
\frac{\mathrm{d} P}{\mathrm{~d} t}= & \frac{\mathrm{d} x}{\mathrm{~d} t}+\frac{\mathrm{d} w}{\mathrm{~d} t}+\frac{1}{c_{1}} \frac{\mathrm{d} y}{\mathrm{~d} t} \\
= & \frac{r x}{1+k y}-d_{1} x-a_{1} x^{2}+A-d_{2} w-\frac{a_{3} w y(a+y)}{\left(b_{1}+w\right)(a+y)+(a+(1-m) y) x} \\
& +\frac{\left(c_{2} / c_{1}\right) a_{3} w y(a+y)}{\left(b_{1}+w\right)(a+y)+(a+(1-m) y) x}-\frac{d_{3} y}{c_{1}} \\
\leq & r x-d_{1} x-a_{1} x^{2}+A-d_{2} w-d_{3} \frac{y}{c_{1}}-\frac{\left(b_{1}+w\right)(a+y)+(a+(1-m) y) x}{a_{3} w y(a+y)}\left(1-\frac{c_{2}}{c_{1}}\right) \\
\leq & 2\left(r-d_{1}\right) x-a_{1} x^{2}+A-\left(r-d_{1}\right) x-d_{2} w-d_{3} \frac{y}{c_{1}}\left[\operatorname{since} c_{1}>c_{2}\right] \\
= & -a_{1}\left(x-\frac{r-d_{1}}{a_{1}}\right)^{2}+\frac{\left(r-d_{1}\right)^{2}}{a_{1}}+A-\left\{\left(r-d_{1}\right) x+d_{2} w+d_{3} \frac{y}{c_{1}}\right\} \\
\leq & \frac{\left(r-d_{1}\right)^{2}}{a_{1}}+A-\left\{\left(r-d_{1}\right) x+d_{2} w+d_{3} \frac{y}{c_{1}}\right\}, \quad \frac{\mathrm{d} P}{\mathrm{~d} t}+\eta P \leq \frac{\left(r-d_{1}\right)^{2}}{a_{1}}+A . \\
\therefore \frac{\mathrm{d} P}{\mathrm{~d} t} \leq & \frac{\left(r-d_{1}\right)^{2}}{a_{1}}+A-\left\{\left(r-d_{1}\right) x+d_{2} w+d_{3} \frac{y}{c_{1}}\right\} .
\end{aligned}
$$

Let

$$
\eta=\min \left\{r-d_{1}, d_{2}, d_{3}\right\}, \quad \text { when } r>d_{1} .
$$

Then,

Using the Gronwall inequality, we obtain

$$
\begin{aligned}
& 0<P(x(t), w(t), y(t)) \leq \frac{\left(\left(r-d_{1}\right)^{2} / a_{1}\right)+A}{\eta}\left(1-e^{-\eta t}\right)+e^{-\eta t} P(x(0), w(0), y(0)), \\
\therefore 0 & <P(x(t), w(t), y(t)) \leq \frac{\left(\left(r-d_{1}\right)^{2} / a_{1}\right)+A}{\eta}, \quad \text { as } t \longrightarrow \infty .
\end{aligned}
$$

Thus, all solutions of system (2) enter into the region:

$$
\Omega=\left\{(x, w, y) \in \mathbb{R}_{+}^{3}: 0<x(t) \leq \frac{r-d_{1}}{a_{1}} ; 0<P(x(t), w(t), y(t)) \leq \frac{\left(\left(r-d_{1}\right)^{2} / a_{1}\right)+A}{\eta}\right\} .
$$

Case 2: if $r<d_{1}$, from the first equation of (2) we obtain $\lim _{t \rightarrow \infty} x(t)=0$.
Now, from the second and third equations of (2), we have

$$
\frac{\mathrm{d} w}{\mathrm{~d} t}+\frac{1}{c_{2}} \frac{\mathrm{d} y}{\mathrm{~d} t}=A-d_{2} w+\frac{\left(c_{1} / c_{2}\right) a_{2}(1-(m y /(a+y))) x y}{b_{1}+w+(1-(m y /(a+y))) x}-d_{3} \frac{y}{c_{2}} .
$$

For large $t$, 
$\frac{\mathrm{d} w}{\mathrm{~d} t}+\frac{1}{c_{2}} \frac{\mathrm{d} y}{\mathrm{~d} t}=A-d_{2} w-d_{3} \frac{y}{c_{2}}\left[\because \lim _{t \rightarrow \infty} x(t)=0\right]$.

Let

$$
\eta^{\prime}=\min \left\{d_{2}, d_{3}\right\}
$$

Then,

$$
\frac{\mathrm{d}}{\mathrm{d} t}\left(w+\frac{y}{c_{2}}\right)+\eta^{\prime}\left(w+\frac{y}{c_{2}}\right) \leq A .
$$

Using Gronwall inequality, we obtain

$$
\begin{aligned}
0<w(t)+\frac{y(t)}{c_{2}} \leq \frac{A}{\eta^{\prime}}\left(1-e^{-\eta^{\prime} t}\right)+e^{-\eta^{\prime} t}\left(w(0)+\frac{y(0)}{c_{2}}\right), \\
\therefore 0<w(t)+\frac{y(t)}{c_{2}} \leq \frac{A}{\eta^{\prime}}, \quad \text { as } t \longrightarrow \infty .
\end{aligned}
$$

Hence, the theorem.

\section{Equilibrium Points and Stability Analysis}

\subsection{Equilibria}

4.1.1. Trivial Equilibrium Point. Extinction: $E_{0}(0,0,0)$.

\subsubsection{Axial Equilibrium Points}

(i) Subsidy only: $E_{1}\left(0,\left(A / d_{2}\right), 0\right)$

(ii) Prey only: $E_{2}\left(\left(r-d_{1}\right) / a_{1}, 0,0\right)$ exists if $r>d_{1}$ and $A=0$

\subsubsection{Planer Equilibrium Points}

(i) Subsidy free: $E_{3}(\bar{x}, 0, \bar{y})$ exists if $A=0, \bar{x}>0$, and $\bar{y}>0$ where $\bar{x}$ and $\bar{y}$ can be obtained by solving the equations:

$$
\begin{aligned}
& \frac{r}{1+k y}-d_{1}-a_{1} x-\frac{a_{2}(1-(m y /(a+y))) y}{b_{1}+(1-(m y /(a+y))) x}=0, \\
& \frac{c_{1} a_{2}(1-(m y /(a+y))) x}{b_{1}+(1-(m y /(a+y))) x}-d_{3}=0,
\end{aligned}
$$

and we get

$$
\bar{y}=\frac{a\left\{d_{3} b_{1}-\bar{x}\left(c_{1} a_{2}-d_{3}\right)\right\}}{(1-m)\left(c_{1} a_{2}-d_{3}\right) \bar{x}-d_{3} b_{1}}, \quad m \neq 1,
$$

where $\bar{x}$ is a positive root of the equation:

$$
B_{0} x^{4}+B_{1} x^{3}+B_{2} x^{2}+B_{3} x+B_{4}=0 .
$$

Here,

$$
\begin{aligned}
B_{0}= & a_{1}(1-m)(1-m-a k)\left(c_{1} a_{2}-d_{3}\right)^{2} \\
B_{1}= & a_{1} b_{1} d_{3}(1-m)(a k-1)\left(c_{1} a_{2}-d_{3}\right)-a_{1} b_{1} d_{3}(1-m-a k)\left(c_{1} a_{2}-d_{3}\right)+d_{1}(1-m)(1-m-a k)\left(c_{1} a_{2}-d_{3}\right)^{2} \\
& -r(1-m)^{2}\left(c_{1} a_{2}-d_{3}\right)^{2} \\
B_{2}= & b_{1} d_{1} d_{3}(1-m)(a k-1)\left(c_{1} a_{2}-d_{3}\right)-a_{1} b_{1}^{2} d_{3}^{2}(a k-1)-b_{1} d_{1} d_{3}(1-m-a k)\left(c_{1} a_{2}-d_{3}\right) \\
& -\frac{d_{3}}{c_{1}}(1-m-a k)\left(c_{1} a_{2}-d_{3}\right)^{2}+2 b_{1} d_{3} r(1-m)\left(c_{1} a_{2}-d_{3}\right) \\
B_{3}= & -b_{1}^{2} d_{3}^{2} r+\frac{b_{1} d_{3}^{2}}{c_{1}}(1-m-a k)\left(c_{1} a_{2}-d_{3}\right)-\frac{b_{1} d_{3}^{2}}{c_{1}}(a k-1)\left(c_{1} a_{2}-d_{3}\right)-b_{1}^{2} d_{1} d_{3}^{2}(a k-1) \\
B_{4}= & \frac{b_{1}^{2} d_{3}^{3}}{c_{1}}(a k-1)
\end{aligned}
$$

and $\quad \bar{y}=\left(a\left\{d_{3} b_{1}-\bar{x}\left(c_{1} a_{2}-d_{3}\right)\right\} /\left((1-m)\left(c_{1}\right.\right.\right.$ $\left.\left.\left.a_{2}-d_{3}\right) \bar{x}-d_{3} d_{1}\right)\right)$ exists if $(1-m)\left(c_{1} a_{2}-\right.$ $\left.d_{3}\right) \bar{x}<d_{3} b_{1}<\left(c_{1} a_{2}-d_{3}\right) \bar{x}$ and $c_{1} a_{2}>d_{3}$.

(ii) Prey free: $E_{4}\left(0, \widehat{w}=\left(d_{3} b_{1} /\left(c_{2} a_{3}-d_{3}\right)\right), \widehat{y}=((A-\right.$ $\left.\left.\left.d_{2} \widehat{w}\right)\left(b_{1}+\widehat{w}\right) / a_{3}\right)\right)$ exists if $c_{2} a_{3}>d_{3}$ and $\hat{y}>0$. (iii) Predator free: $E_{5}\left(\left(r-d_{1}\right) / a_{1},\left(A / d_{2}\right), 0\right)$ exists if $r>d_{1}$.

4.1.4. Interior (Coexistence) Equilibrium Point. Solving the following system of equations, 


$$
\begin{array}{r}
\frac{r}{1+k y}-d_{1}-a_{1} x-\frac{a_{2}(1-(m y /(a+y))) y}{b_{1}+w+(1-(m y /(a+y))) x}=0, \\
A-d_{2} w-\frac{a_{3} w y}{b_{1}+w+(1-(m y /(a+y))) x}=0, \\
\frac{c_{1} a_{2}(1-(m y /(a+y))) x}{b_{1}+w+(1-(m y /(a+y))) x}+\frac{c_{2} a_{3} w}{b_{1}+w+(1-(m y /(a+y))) x}-d_{3}=0,
\end{array}
$$

we can obtain $E^{*}\left(x^{*}, w^{*}, y^{*}\right)$ using the software MATHEMATICA with the following existence conditions:

(1) $d_{3}<\min \left\{c_{1} a_{2}, c_{2} a_{3}\right\}$

(2) $0<w^{*}<\min \left\{\left(A / d_{2}\right), d_{3} b_{1} /\left(c_{2} a_{3}-d_{3}\right)\right\}$

(3) $r>d_{1}+a_{1} x^{*}+\left(\left\{b_{1} d_{3}-\left(c_{2} a_{3}-d_{3}\right) w^{*}\right\}(A-\right.$ $\left.d_{2} w^{*}\right) / a_{3} x^{*} w^{*}\left(c_{1} a_{2}-d_{3}\right)$ ) (otherwise, predator population goes into extinction)

4.2. Stability Analysis. Now, we will study the stability conditions of all equilibria for the proposed system (2).

The Jacobian matrix $J_{0}(0,0,0)$ at $E_{0}(0,0,0)$ is given by

$$
J_{0}(0,0,0)=\left[\begin{array}{ccc}
r-d_{1} & 0 & 0 \\
0 & -d_{2} & 0 \\
0 & 0 & -d_{3}
\end{array}\right] .
$$

The eigenvalues of $J_{0}(0,0,0)$ are $r-d_{1},-d_{2}(<0)$, and $-d_{3}(<0)$. Then. we have stated the following theorem.

Theorem 3. Trivial equilibrium point $E_{0}(0,0,0)$ is locally asymptotically stable (LAS) if $r<d_{1}$ and unstable if $r>d_{1}$.

The Jacobian matrix $J_{1}\left(0,\left(A / d_{2}\right), 0\right)$ at $E_{1}\left(0,\left(A / d_{2}\right), 0\right)$ is as follows:

$$
J_{1}\left(0, \frac{A}{d_{2}}, 0\right)=\left[\begin{array}{ccc}
r-d_{1} & 0 & 0 \\
0 & -d_{2} & -\frac{a_{3}\left(A / d_{2}\right)}{b_{1}+\left(A / d_{2}\right)} \\
0 & 0 & -d_{3}+\frac{c_{2} a_{3}\left(A / d_{2}\right)}{b_{1}+\left(A / d_{2}\right)}
\end{array}\right]
$$

We see that

$$
\begin{aligned}
& r-d_{1}, \\
& -d_{2}(<0), \\
& -d_{3}+\frac{c_{2} a_{3}\left(A / d_{2}\right)}{b_{1}+\left(A / d_{2}\right)},
\end{aligned}
$$

are the eigenvalues of the matrix $J_{1}\left(0,\left(A / d_{2}\right), 0\right)$. Thus, we have the following theorem.

Theorem 4. Axial equilibrium point (subsidy only) $E_{1}\left(0,\left(A / d_{2}\right), 0\right)$ is locally asymptotically stable if $r<d_{1}$ and $\left(c_{2} a_{3}\left(A / d_{2}\right) / b_{1}+\left(A / d_{2}\right)\right)<d_{3}$ and unstable if either $\left\{r>d_{1},\left(c_{2} a_{3}\left(A / d_{2}\right) / b_{1}+\left(A / d_{2}\right)\right)<d_{3}\right\} \quad$ or $\quad\left\{r<d_{1},\left(c_{2} a_{3}\right.\right.$ $\left.\left.\left(A / d_{2}\right) / b_{1}+\left(A / d_{2}\right)\right)>d_{3}\right\} \quad$ or $\quad\left\{r>d_{1},\left(c_{2} a_{3}\left(A / d_{2}\right) / b_{1}+\right.\right.$ $\left.\left.\left(A / d_{2}\right)\right)>d_{3}\right\}$

The Jacobian matrix $J_{2}\left(\left(r-d_{1}\right) / a_{1}, 0,0\right)$ at $E_{2}\left(\left(r-d_{1}\right) / a_{1}, 0,0\right)$ is given by

$$
J_{2}\left(\frac{r-d_{1}}{a_{1}}, 0,0\right)=\left[\begin{array}{ccc}
-r+d_{1} & 0 & \frac{r-d_{1}}{a_{1}}\left(-r k-\frac{a_{2}}{b_{1}+\left(\left(r-d_{1}\right) / a_{1}\right)}\right) \\
0 & -d_{2} & 0 \\
0 & 0 & -d_{3}+\frac{c_{1} a_{2}\left(\left(r-d_{1}\right) / a_{1}\right)}{b_{1}+\left(\left(r-d_{1}\right) / a_{1}\right)}
\end{array}\right]
$$


The eigenvalues of $J_{2}\left(\left(r-d_{1}\right) / a_{1}, 0,0\right)$ are $-r+d_{1}(<0)$, $-d_{2}(<0)$ or $-d_{3}+\left(c_{1} a_{2}\left(\left(r-d_{1}\right) / a_{1}\right) / b_{1}+\left(\left(r-d_{1}\right) / a_{1}\right)\right)$. Thus. we have stated the following theorem.
Theorem 5. Axial equilibrium point (prey only) $E_{2}((r-$ $\left.\left.d_{1}\right) / a_{1}, 0,0\right)$ is locally asymptotically stable if

$$
\frac{c_{1} a_{2}\left(\left(r-d_{1}\right) / a_{1}\right)}{b_{1}+\left(\left(r-d_{1}\right) / a_{1}\right)}<d_{3} \Longrightarrow 0<r<d_{1}+a_{1}\left(\frac{b_{1} d_{3}}{c_{1} a_{2}-d_{3}}\right) \text { with } c_{1} a_{2}>d_{3}
$$

If $r>d_{1}+a_{1}\left(b_{1} d_{3} /\left(c_{1} a_{2}-d_{3}\right)\right)$ with $c_{1} a_{2}>d_{3}$, then $E_{2}\left(\left(r-d_{1}\right) / a_{1}, 0,0\right)$ is unstable.

$$
J_{3}(\bar{x}, 0, \bar{y})=\left[\begin{array}{lll}
b_{11} & b_{12} & b_{13} \\
b_{21} & b_{22} & b_{23} \\
b_{31} & b_{32} & b_{33}
\end{array}\right],
$$

The Jacobian matrix $J_{3}(\bar{x}, 0, \bar{y})$ at $E_{3}(\bar{x}, 0, \bar{y})$ is as follows:

where

$$
\begin{aligned}
& b_{11}=\bar{x}\left\{-a_{1}+\frac{a_{2} \bar{y}(a+(1-m) \bar{y})^{2}}{\left\{b_{1}(a+\bar{y})+[a+(1-m) \bar{y}]_{\bar{x}}\right\}^{2}}\right\}, \\
& b_{12}=\frac{a_{2} \overline{x y}[a+(1-m) \bar{y}](a+\bar{y})}{\left\{b_{1}(a+\bar{y})+[a+(1-m) \bar{y}] \bar{x}\right\}^{2}}, \\
& b_{13}=\bar{x}\left\{-\frac{r k}{(1+k \bar{y})^{2}}-\frac{a_{2}[a+2(1-m) \bar{y}]}{b_{1}(a+\bar{y})+[a+(1-m) \bar{y}] \bar{x}}+\frac{a_{2} \bar{y}[a+(1-m) \bar{y}]\left[b_{1}+(1-m) \bar{x}\right]}{\left\{b_{1}(a+\bar{y})+[a+(1-m) \bar{y}] \bar{x}\right\}^{2}}\right\}, \\
& b_{21}=0, \\
& b_{22}=-d_{2}-\frac{a_{3} \bar{y}}{b_{1}+(1-(m \bar{y} /(a+\bar{y}))) \bar{x}}, \\
& b_{23}=0, \\
& b_{31}=\bar{y}\left\{\frac{c_{1} a_{2}[a+(1-m) \bar{y}]}{b_{1}(a+\bar{y})+[a+(1-m) \bar{y}] \bar{x}}-\frac{c_{1} a_{2}[a+(1-m) \bar{y}]^{2} \bar{x}}{\left\{b_{1}(a+\bar{y})+[a+(1-m) \bar{y}] \bar{x}\right\}^{2}}\right\}, \\
& b_{32}=\frac{c_{2} a_{3} \bar{y}(a+\bar{y})}{b_{1}\left(a+y^{*}\right)+[a+(1-m) \bar{y}] \bar{x}}, \\
& b_{33}=\bar{y}\left\{\frac{c_{1} a_{2}(1-m) \bar{x}}{b_{1}(a+\bar{y})+[a+(1-m) \bar{y}] \bar{x}}-\frac{c_{1} a_{2}[a+(1-m) \bar{y}] \bar{x}\left[b_{1}+(1-m) \bar{x}\right]}{\left\{b_{1}(a+\bar{y})+[a+(1-m) \bar{y}] \bar{x}\right\}^{2}}\right\} .
\end{aligned}
$$

The characteristic equation corresponding to $J_{3}(\bar{x}, 0, \bar{y})$ is expressed as

$$
\lambda^{3}+B_{1} \lambda^{2}+B_{2} \lambda+B_{3}=0
$$

where $\quad B_{1}=-\left(b_{11}+b_{22}+b_{33}\right), \quad B_{2}=b_{22} b_{33}+b_{11} b_{33}-$ $b_{13} b_{31}+b_{11} b_{22}$, and $B_{3}=-b_{22}\left(b_{11} b_{33}-b_{13} b_{31}\right)$.

Theorem 6. Subsidy-free equilibrium point $E_{3}(\bar{x}, 0, \bar{y})$ is locally asymptotically stable if $b_{11}<0, b_{33}<0$, and $b_{13}<0$.
The Jacobian matrix $J_{4}(0, \widehat{w}, \widehat{y})$ corresponding to $E_{4}(0, \widehat{w}, \widehat{y})$ is given by

$$
J_{4}(0, \widehat{w}, \hat{y})=\left[\begin{array}{lll}
c_{11} & c_{12} & c_{13} \\
c_{21} & c_{22} & c_{23} \\
c_{31} & c_{32} & c_{33}
\end{array}\right],
$$

where 


$$
\begin{aligned}
& c_{11}=\frac{r}{1+k \hat{y}}-d_{1}-\frac{a_{2}[a+(1-m) \hat{y}] \hat{y}}{\left(b_{1}+\widehat{w}\right)(a+\hat{y})}, \\
& c_{12}=0, \\
& c_{13}=0, \\
& c_{21}=\frac{a_{3}(1-m)(a+\hat{y}) \hat{w} \hat{y}^{2}}{\left[\left(b_{1}+\widehat{w}\right)(a+\hat{y})\right]^{2}}, \\
& c_{22}=-d_{2}-\frac{a_{3} \hat{y}(a+\hat{y})}{\left(b_{1}+\widehat{w}\right)(a+\hat{y})}, \\
& c_{23}=-\frac{a_{3} \widehat{w}(a+2 \hat{y})}{\left(b_{1}+\widehat{w}\right)(a+\hat{y})}+\frac{a_{3} \hat{y} \widehat{w}(a+\hat{y})\left(b_{1}+\widehat{w}\right)}{\left[\left(b_{1}+\widehat{w}\right)(a+\hat{y})\right]^{2}}, \\
& c_{31}=\frac{c_{1} a_{2} \hat{y}[a+(1-m) \hat{y}]}{\left(b_{1}+\widehat{w}\right)(a+\hat{y})}, \\
& c_{32}=\frac{c_{2} a_{3} \hat{y}(a+\hat{y})}{\left(b_{1}+\widehat{w}\right)(a+\hat{y})}-\frac{c_{2} a_{3} \hat{w} \hat{y}(a+\hat{y})^{2}}{\left[\left(b_{1}+\widehat{w}\right)(a+\hat{y})\right]^{2}}=\frac{c_{2} a_{3} \hat{y} b_{1}}{\left[\left(b_{1}+\widehat{w}\right)(a+\hat{y})\right]^{2}}, \\
& c_{33}=\frac{c_{2} a_{3} \hat{w} \hat{y}}{\left(b_{1}+\widehat{w}\right)(a+\hat{y})}-\frac{c_{2} a_{3} \widehat{w} \hat{y}(a+\hat{y})\left(b_{1}+\widehat{w}\right)}{\left[\left(b_{1}+\widehat{w}\right)(a+\hat{y})\right]^{2}}=0 .
\end{aligned}
$$

The characteristic equation corresponding to $J_{4}(0, \widehat{w}, \widehat{y})$ is expressed as

$$
\lambda^{3}+C_{1} \lambda^{2}+C_{2} \lambda+C_{3}=0,
$$

where

$$
\begin{aligned}
& C_{1}=-\left(c_{11}+c_{22}\right), \\
& C_{2}=-c_{23} c_{32}+c_{11} c_{22}, \\
& C_{3}=+c_{11} c_{23} c_{32} .
\end{aligned}
$$

Theorem 7. Prey-free equilibrium point $E_{4}(0, \widehat{w}, \widehat{y})$ is locally asymptotically stable if $c_{11}<0$ and $c_{23}<0$.

The Jacobian matrix $J_{5}\left(\left(r-d_{1}\right) / a_{1},\left(A / d_{2}\right), 0\right)$ corresponding to $E_{5}\left(\left(r-d_{1}\right) / a_{1},\left(A / d_{2}\right), 0\right)$ is given by

$$
J_{5}\left(\frac{r-d_{1}}{a_{1}}, \frac{A}{d_{2}}, 0\right)=\left[\begin{array}{lll}
d_{11} & d_{12} & d_{13} \\
d_{21} & d_{22} & d_{23} \\
d_{31} & d_{32} & d_{33}
\end{array}\right],
$$

where

$$
\begin{aligned}
& d_{11}=-r+d_{1}, \\
& d_{12}=0, \\
& d_{13}=\frac{r-d_{1}}{a_{1}}\left[-r k-\frac{a_{2}}{b_{1}+\left(A / d_{2}\right)+\left(\left(r-d_{1}\right) / a_{1}\right)}\right], \\
& d_{21}=0, \\
& d_{22}=-d_{2}, \\
& d_{23}=-\frac{a_{3}\left(A / d_{2}\right)}{b_{1}+\left(A / d_{2}\right)+\left(\left(r-d_{1}\right) / a_{1}\right)}, \\
& d_{31}=d_{32}=0, \\
& d_{33}=\frac{c_{1} a_{2}\left(\left(r-d_{1}\right) / a_{1}\right)}{b_{1}+\left(A / d_{2}\right)+\left(\left(r-d_{1}\right) / a_{1}\right)}+\frac{c_{2} a_{3}\left(A / d_{2}\right)}{b_{1}+\left(A / d_{2}\right)+\left(\left(r-d_{1}\right) / a_{1}\right)}-d_{3} .
\end{aligned}
$$

The characteristic equation corresponding to $J_{5}\left(\left(r-d_{1}\right) / a_{1},\left(A / d_{2}\right), 0\right)$ is expressed as

$$
\lambda^{3}+D_{1} \lambda^{2}+D_{2} \lambda+D_{3}=0,
$$

where

$$
\begin{aligned}
& D_{1}=-\left(d_{11}+d_{22}+d_{33}\right), \\
& D_{2}=d_{22} d_{33}+d_{11} d_{33}+d_{11} d_{22}, \\
& D_{3}=-d_{11} d_{22} d_{33} .
\end{aligned}
$$


Theorem 8. Predator free equilibrium point $E_{5}\left(\left(r-d_{1}\right) /\right.$ $\left.a_{1},\left(A / d_{2}\right), 0\right)$ is locally asymptotically stable if

$$
\frac{c_{1} a_{2}\left(\left(r-d_{1}\right) / a_{1}\right)}{b_{1}+\left(A / d_{2}\right)+\left(\left(r-d_{1}\right) / a_{1}\right)}+\frac{c_{2} a_{3}\left(A / d_{2}\right)}{b_{1}+\left(A / d_{2}\right)+\left(\left(r-d_{1}\right) / a_{1}\right)}<d_{3} \Longrightarrow 0<r<d_{1}+a_{1}\left\{\frac{b_{1} d_{3}-\left(c_{2} a_{3}-d_{3}\right)\left(A / d_{2}\right)}{c_{1} a_{2}-d_{3}}\right\}
$$

provided $d_{3}<\min \left\{c_{1} a_{2}, c_{2} a_{3}\right\} \quad$ and $0<\left(A / d_{2}\right)<\left(b_{1} d_{3}\right.$ $\left./\left(c_{2} a_{3}-d_{3}\right)\right)$.

$$
J^{*}\left(x^{*}, w^{*}, y^{*}\right)=\left[\begin{array}{lll}
a_{11} & a_{12} & a_{13} \\
a_{21} & a_{22} & a_{23} \\
a_{31} & a_{32} & a_{33}
\end{array}\right]
$$

The Jacobian matrix $J^{*}\left(x^{*}, w^{*}, y^{*}\right)$ corresponding to $E^{*}\left(x^{*}, w^{*}, y^{*}\right)$ is as follows:

where

$$
\begin{aligned}
& a_{11}=x^{*}\left[-a_{1}+\frac{a_{2}\left(a+(1-m) y^{*}\right)^{2} y^{*}}{\left[\left(b_{1}+w^{*}\right)\left(a+y^{*}\right)+\left(a+(1-m) y^{*}\right) x^{*}\right]^{2}}\right] \\
& a_{12}=\frac{a_{2}\left(a+(1-m) y^{*}\right)\left(a+y^{*}\right) x^{*} y^{*}}{\left[\left(b_{1}+w^{*}\right)\left(a+y^{*}\right)+\left(a+(1-m) y^{*}\right) x^{*}\right]^{2}} \\
& a_{13}=\left[-\frac{r k x^{*}}{\left(1+k y^{*}\right)^{2}}-\frac{a_{2}\left(a+2(1-m) y^{*}\right) x^{*}}{\left(b_{1}+w^{*}\right)\left(a+y^{*}\right)+\left(a+(1-m) y^{*}\right) x^{*}}+\frac{a_{2}\left(a+(1-m) y^{*}\right) x^{*} y^{*}\left(b_{1}+w^{*}+(1-m) x^{*}\right)}{\left[\left(b_{1}+w^{*}\right)\left(a+y^{*}\right)+\left(a+(1-m) y^{*}\right) x^{*}\right]^{2}}\right] \text {, } \\
& a_{21}=\frac{a_{3}\left(a+y^{*}\right)\left(a+(1-m) y^{*}\right) w^{*} y^{*}}{\left[\left(b_{1}+w^{*}\right)\left(a+y^{*}\right)+\left(a+(1-m) y^{*}\right) x^{*}\right]^{2}} \\
& a_{22}=-d_{2}-\frac{a_{3}\left(a+y^{*}\right) y^{*}}{\left(b_{1}+w^{*}\right)\left(a+y^{*}\right)+\left(a+(1-m) y^{*}\right) x^{*}}+\frac{a_{3}\left(a+y^{*}\right)^{2} w^{*} y^{*}}{\left[\left(b_{1}+w^{*}\right)\left(a+y^{*}\right)+\left(a+(1-m) y^{*}\right) x^{*}\right]^{2}}, \\
& a_{23}=-\frac{a_{3}\left(a+2 y^{*}\right) w^{*}}{\left(b_{1}+w^{*}\right)\left(a+y^{*}\right)+\left(a+(1-m) y^{*}\right) x^{*}}+\frac{a_{3}\left(a+y^{*}\right)\left(b_{1}+w^{*}+(1-m) x^{*}\right)}{\left[\left(b_{1}+w^{*}\right)\left(a+y^{*}\right)+\left(a+(1-m) y^{*}\right) x^{*}\right]^{2}}, \\
& a_{31}=\frac{c_{1} a_{2}\left(a+(1-m) y^{*}\right) y^{*}}{\left(b_{1}+w^{*}\right)\left(a+y^{*}\right)+\left(a+(1-m) y^{*}\right) x^{*}}-\frac{c_{1} a_{2}\left(a+(1-m) y^{*}\right)^{2} x^{*} y^{*}}{\left[\left(b_{1}+w^{*}\right)\left(a+y^{*}\right)+\left(a+(1-m) y^{*}\right) x^{*}\right]^{2}} \\
& -\frac{c_{2} a_{3} w^{*} y^{*}\left(a+y^{*}\right)\left(a+(1-m) y^{*}\right)}{\left[\left(b_{1}+w^{*}\right)\left(a+y^{*}\right)+\left(a+(1-m) y^{*}\right) x^{*}\right]^{2}} \\
& a_{32}=-\frac{c_{1} a_{2}\left(a+(1-m) y^{*}\right) x^{*} y^{*}\left(a+y^{*}\right)}{\left[\left(b_{1}+w^{*}\right)\left(a+y^{*}\right)+\left(a+(1-m) y^{*}\right) x^{*}\right]^{2}}+\frac{c_{2} a_{3}\left(a+y^{*}\right) y^{*}}{\left(b_{1}+w^{*}\right)\left(a+y^{*}\right)+\left(a+(1-m) y^{*}\right) x^{*}} \\
& -\frac{c_{2} a_{3} w^{*} y^{*}\left(a+y^{*}\right)^{2}}{\left[\left(b_{1}+w^{*}\right)\left(a+y^{*}\right)+\left(a+(1-m) y^{*}\right) x^{*}\right]^{2}} \\
& a_{33}=\frac{c_{1} a_{2}(1-m) x^{*} y^{*}+c_{2} a_{3} w^{*} y^{*}}{\left(b_{1}+w^{*}\right)\left(a+y^{*}\right)+\left(a+(1-m) y^{*}\right) x^{*}}-\frac{\left[c_{1} a_{2}\left(a+(1-m) y^{*}\right) x^{*} y^{*}+c_{2} a_{3} w^{*} y^{*}\left(a+y^{*}\right)\right]\left(b_{1}+w^{*}+(1-m) x^{*}\right)}{\left[\left(b_{1}+w^{*}\right)\left(a+y^{*}\right)+\left(a+(1-m) y^{*}\right) x^{*}\right]^{2}}
\end{aligned}
$$

The characteristic equation corresponding to $J^{*}\left(x^{*}, w^{*}, y^{*}\right)$ is expressed as

$$
\lambda^{3}+A_{1} \lambda^{2}+A_{2} \lambda+A_{3}=0,
$$

where 


$$
\begin{aligned}
& A_{1}=-\left(a_{11}+a_{22}+a_{33}\right), \\
& A_{2}=a_{22} a_{33}-a_{23} a_{32}+a_{11} a_{33}-a_{13} a_{31}+a_{11} a_{22}-a_{12} a_{21}, \\
& A_{3}=-\left[a_{11}\left(a_{22} a_{33}-a_{23} a_{32}\right)+a_{12}\left(a_{23} a_{31}-a_{21} a_{33}\right)+a_{13}\left(a_{21} a_{32}-a_{22} a_{31}\right)\right] .
\end{aligned}
$$

Theorem 9. The coexistence equilibrium $E^{*}\left(x^{*}, w^{*}, y^{*}\right)$ is locally asymptotically stable if $A_{1}>0, A_{3}>0$, and $A_{1} A_{2}>A_{3}$, where $A_{1}, A_{2}$, and $A_{3}$ are stated in (41).

\subsection{Local Bifurcations of Codimension 1}

\subsubsection{Transcritical Bifurcation}

Theorem 10. System (2) undergoes a transcritical bifurcation around $E_{1}\left(0,\left(A / d_{2}\right), 0\right)$ if $d_{1}^{[T C]}=r$ and $\left(c_{2} a_{3}\left(A / d_{2}\right) /\left(b_{1}+\left(A / d_{2}\right)\right)\right)<d_{3} \quad([T C]$ stands for transcritical bifurcation).
Proof. We apply Sotomayor's theorem [43] to prove the occurrence of a transcritical bifurcation around $E_{1}$ with $d_{1}$ as bifurcation parameter. For applicability of Sotomayor's theorem, exactly one of the eigenvalues of the Jacobian matrix at $E_{1}$ must be zero and other eigenvalues must have negative real parts. So, we need to fulfill the condition $\left(c_{2} a_{3}\left(A / d_{2}\right) /\left(b_{1}+\left(A / d_{2}\right)\right)\right)<d_{3}$.

The eigenvectors of $J\left(E_{1}\right)=\left[v_{i j}\right]$ and $\left(J\left(E_{1}\right)\right)^{T}$ corresponding to the zero eigenvalue of $E_{1}\left(0,\left(A / d_{2}\right), 0\right)$ are obtained as $V=\left(v_{1}, v_{2}, v_{3}\right)^{T}$ and $W=(1,0,0)^{T}$, respectively, where $v_{1}=1, v_{2}=v_{3}=0, v_{11}=0, v_{12}=0, v_{13}=0$, $v_{21}=0, \quad v_{22}=-d_{2}, \quad v_{23}=0, \quad v_{31}=0, \quad v_{32}=0$, $v_{33}=-d_{3}+\left(c_{2} a_{3}\left(A / d_{2}\right) /\left(b_{1}+\left(A / d_{2}\right)\right)\right)$.

Compute $\Delta_{1}, \Delta_{2}$, and $\Delta_{3}$ as follows:

$$
\Delta_{1}=W^{T} \cdot F_{d_{1}}\left(0, \frac{A}{d_{2}}, 0 ; d_{1}^{[\mathrm{TC}]}\right)=(1,0,0) \cdot\left(\begin{array}{c}
\frac{\partial F_{1}}{\partial d_{1}} \\
\frac{\partial F_{2}}{\partial d_{1}} \\
\frac{\partial F_{3}}{\partial d_{1}}
\end{array}\right)_{E_{1}}=(1,0,0) \cdot\left(\begin{array}{c}
-x \\
0 \\
0
\end{array}\right)_{E_{1}}=0
$$

where $F=\left(F_{1}, F_{2}, F_{3}\right)^{T}$ and $F_{1}, F_{2}$, and $F_{3}$ are given by

$$
\begin{aligned}
& F_{1}=\frac{r x}{1+k y}-d_{1} x-a_{1} x^{2}-\frac{a_{2}(1-(m y /(a+y))) x y}{b_{1}+w+(1-(m y /(a+y))) x}, \\
& F_{2}=A-d_{2} w-\frac{a_{3} w y}{b_{1}+w+(1-(m y /(a+y))) x}, \\
& F_{3}=\frac{c_{1} a_{2}(1-(m y /(a+y))) x y}{b_{1}+w+(1-(m y /(a+y))) x}+\frac{c_{2} a_{3} w y}{b_{1}+w+(1-(m y /(a+y))) x}-d_{3} y, \\
& \Delta_{2}=W^{T} \cdot\left[D F_{d_{1}}\left(0, \frac{A}{d_{2}}, 0 ; d_{1}^{[\mathrm{TC}]}\right) V\right]=(1,0,0) \cdot\left[\begin{array}{l}
\frac{\partial^{2} F_{1}}{\partial x \partial d_{1}} \frac{\partial^{2} F_{1}}{\partial w \partial d_{1}} \frac{\partial^{2} F_{1}}{\partial y \partial d_{1}} \\
\frac{\partial^{2} F_{2}}{\partial x \partial d_{1}} \frac{\partial^{2} F_{2}}{\partial w \partial d_{1}} \frac{\partial^{2} F_{2}}{\partial y \partial d_{1}} \\
\left.\frac{\partial^{2} F_{3}}{\partial x \partial d_{1}} \frac{\partial^{2} F_{3}}{\partial w \partial d_{1}} \frac{\partial^{2} F_{3}}{\partial y \partial d_{1}}\right]_{E_{1}} \cdot\left(\begin{array}{l}
1 \\
0 \\
0
\end{array}\right)
\end{array}\right.
\end{aligned}
$$




$$
\begin{aligned}
& =(1,0,0) \cdot\left[\begin{array}{ccc}
-1 & 0 & 0 \\
0 & 0 & 0 \\
0 & 0 & 0
\end{array}\right]_{E_{1}} \cdot\left(\begin{array}{l}
1 \\
0 \\
0
\end{array}\right)=-1 \neq 0, \\
& \Delta_{3}=W^{T} \cdot\left[D^{2} F\left(0, \frac{A}{d_{2}}, 0 ; d_{1}^{[\mathrm{TC}]}\right)(V, V)\right]=(1,0,0) \cdot D\left(\begin{array}{c}
\frac{\partial F_{1}}{\partial x} v_{1}+\frac{\partial F_{1}}{\partial w} v_{2}+\frac{\partial F_{1}}{\partial y} v_{3} \\
\frac{\partial F_{2}}{\partial x} v_{1}+\frac{\partial F_{2}}{\partial w} v_{2}+\frac{\partial F_{2}}{\partial y} v_{3} \\
\frac{\partial F_{3}}{\partial x} v_{1}+\frac{\partial F_{3}}{\partial w} v_{2}+\frac{\partial F_{3}}{\partial y} v_{3}
\end{array}\right)_{E_{1}} \cdot\left(\begin{array}{c}
v_{1} \\
v_{2} \\
v_{3}
\end{array}\right) \\
& =(1,0,0) \cdot\left(\begin{array}{l}
\frac{\partial^{2} F_{1}}{\partial x^{2}} v_{1}^{2}+\frac{\partial^{2} F_{1}}{\partial w^{2}} v_{2}^{2}+\frac{\partial^{2} F_{1}}{\partial y^{2}} v_{3}^{2}+2 \frac{\partial^{2} F_{1}}{\partial x \partial w} v_{1} v_{2}+2 \frac{\partial^{2} F_{1}}{\partial x \partial y} v_{1} v_{3}+2 \frac{\partial^{2} F_{1}}{\partial w \partial y} v_{2} v_{3} \\
\frac{\partial^{2} F_{2}}{\partial x^{2}} v_{1}^{2}+\frac{\partial^{2} F_{2}}{\partial w^{2}} v_{2}^{2}+\frac{\partial^{2} F_{2}}{\partial y^{2}} v_{3}^{2}+2 \frac{\partial^{2} F_{2}}{\partial x \partial w} v_{1} v_{2}+2 \frac{\partial^{2} F_{2}}{\partial x \partial y} v_{1} v_{3}+2 \frac{\partial^{2} F_{2}}{\partial w \partial y} v_{2} v_{3} \\
\frac{\partial^{2} F_{3}}{\partial x^{2}} v_{1}^{2}+\frac{\partial^{2} F_{3}}{\partial w^{2}} v_{2}^{2}+\frac{\partial^{2} F_{3}}{\partial y^{2}} v_{3}^{2}+2 \frac{\partial^{2} F_{3}}{\partial x \partial w} v_{1} v_{2}+2 \frac{\partial^{2} F_{3}}{\partial x \partial y} v_{1} v_{3}+2 \frac{\partial^{2} F_{3}}{\partial w \partial y} v_{2} v_{3}
\end{array}\right)_{E_{1}} \\
& =-2 a_{1} \neq 0 \text {. }
\end{aligned}
$$

Therefore, by Sotomayor's theorem [43], system (2) undergoes a transcritical bifurcation at $d_{1}=d_{1}^{[\mathrm{TC}]}$ around the axial equilibrium point $E_{1}$.

Theorem 11. System (2) exhibits a transcritical bifurcation around $E_{2}\left(\left(r-d_{1}\right) / a_{1}, 0,0\right)$ if

$$
d_{3}^{[\mathrm{TC}]}=\frac{c_{1} a_{2}\left(\left(r-d_{1}\right) / a_{1}\right)}{b_{1}+\left(\left(r-d_{1}\right) / a_{1}\right)} .
$$

Proof. Let us apply Sotomayor's theorem [43] to prove the occurrence of a transcritical bifurcation around $E_{2}$ with $d_{3}$ as bifurcation parameter. For applicability of Sotomayor's theorem, exactly one of the eigenvalues of the Jacobian matrix at $E_{2}$ must be zero and other eigenvalues must have negative real parts.

The eigenvectors of $J\left(E_{2}\right)=\left[t_{i j}\right]$ and $\left(J\left(E_{2}\right)\right)^{T}$ corresponding to the zero eigenvalue of $E_{2}\left(\left(r-d_{1}\right) / a_{1}, 0,0\right)$ are obtained as $V=\left(v_{1}, v_{2}, v_{3}\right)^{T}$ and $W=(0,0,1)^{T}$, respectively, where $\quad v_{1}=\left(1 / a_{1}\right)\left[-r k-\left(a_{2} /\left(b_{1}+((r-\right.\right.\right.$ $\left.\left.\left.\left.\left.d_{1}\right) / a_{1}\right)\right)\right)\right]<0, \quad v_{2}=0, \quad v_{3}=1, \quad t_{11}=-r+d_{1}, \quad t_{12}=0$, $t_{13}=\left(\left(r-d_{1}\right) / a_{1}\right)\left[-r k-\left(a_{2} /\left(b_{1}+\left(\left(r-d_{1}\right) / a_{1}\right)\right)\right)\right], \quad t_{21}=$ $0, t_{22}=-d_{2}, t_{23}=0, t_{31}=0, t_{32}=0, t_{33}=0$.

Compute $\Delta_{1}, \Delta_{2}$, and $\Delta_{3}$ as follows:

$$
\Delta_{1}=W^{T} \cdot F_{d_{1}}\left(\frac{r-d_{1}}{a_{1}}, 0,0 ; d_{3}^{[\mathrm{TC}]}\right)=(0,0,1) \cdot\left(\begin{array}{c}
\frac{\partial F_{1}}{\partial d_{3}} \\
\frac{\partial F_{2}}{\partial d_{3}} \\
\frac{\partial F_{3}}{\partial d_{3}}
\end{array}\right)_{E_{2}}=(0,0,1) \cdot\left(\begin{array}{c}
0 \\
0 \\
-y
\end{array}\right)_{E_{2}}=0
$$

where $F=\left(F_{1}, F_{2}, F_{3}\right)^{T}$ and $F_{1}, F_{2}$, and $F_{3}$ are given by 


$$
\begin{aligned}
& F_{1}=\frac{r x}{1+k y}-d_{1} x-a_{1} x^{2}-\frac{a_{2}(1-(m y /(a+y))) x y}{b_{1}+w+(1-(m y /(a+y))) x} \\
& F_{2}=A-d_{2} w-\frac{a_{3} w y}{b_{1}+w+(1-(m y /(a+y))) x}, \\
& F_{3}=\frac{c_{1} a_{2}(1-(m y /(a+y))) x y}{b_{1}+w+(1-(m y /(a+y))) x}+\frac{c_{2} a_{3} w y}{b_{1}+w+(1-(m y /(a+y))) x}-d_{3} y,
\end{aligned}
$$

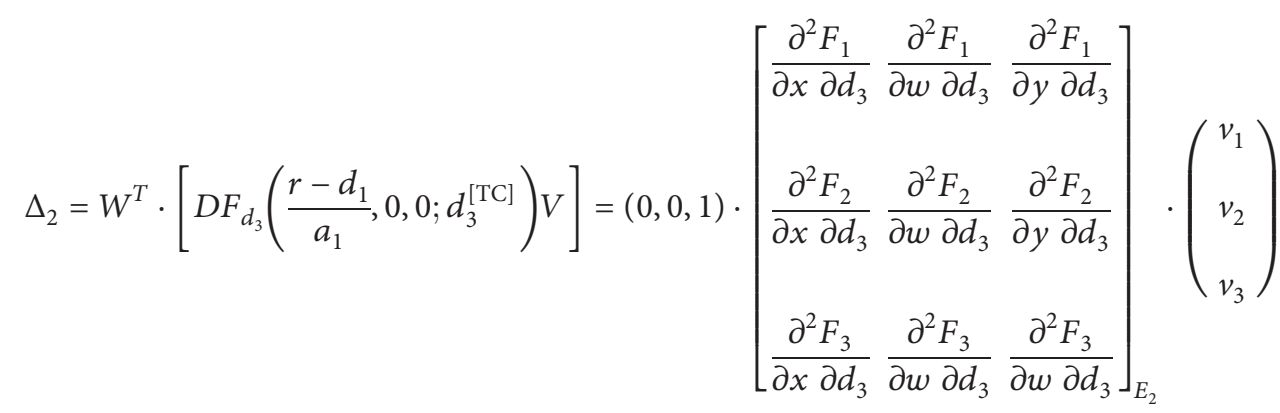

$$
\begin{aligned}
& =(0,0,1) \cdot\left[\begin{array}{ccc}
0 & 0 & 0 \\
0 & 0 & 0 \\
0 & 0 & -1
\end{array}\right]_{E_{2}} \cdot\left(\begin{array}{c}
v_{1} \\
v_{2} \\
v_{3}
\end{array}\right)=-v_{3}=-1 \neq 0, \\
& \Delta_{3}=W^{T} \cdot\left[D^{2} F\left(\frac{r-d_{1}}{a_{1}}, 0,0 ; d_{3}^{[\mathrm{TC}]}\right)(V, V)\right]=(0,0,1) \cdot D\left(\begin{array}{l}
\frac{\partial F_{1}}{\partial x} v_{1}+\frac{\partial F_{1}}{\partial w} v_{2}+\frac{\partial F_{1}}{\partial y} v_{3} \\
\frac{\partial F_{2}}{\partial x} v_{1}+\frac{\partial F_{2}}{\partial w} v_{2}+\frac{\partial F_{2}}{\partial y} v_{3} \\
\frac{\partial F_{3}}{\partial x} v_{1}+\frac{\partial F_{3}}{\partial w} v_{2}+\frac{\partial F_{3}}{\partial y} v_{3}
\end{array}\right)_{E_{2}} \cdot\left(\begin{array}{c}
v_{1} \\
v_{2} \\
v_{3}
\end{array}\right) \\
& =(0,0,1) \cdot\left(\begin{array}{l}
\frac{\partial^{2} F_{1}}{\partial x^{2}} v_{1}^{2}+\frac{\partial^{2} F_{1}}{\partial w^{2}} v_{2}^{2}+\frac{\partial^{2} F_{1}}{\partial y^{2}} v_{3}^{2}+2 \frac{\partial^{2} F_{1}}{\partial x \partial w} v_{1} v_{2}+2 \frac{\partial^{2} F_{1}}{\partial x \partial y} v_{1} v_{3}+2 \frac{\partial^{2} F_{1}}{\partial w \partial y} v_{2} v_{3} \\
\frac{\partial^{2} F_{2}}{\partial x^{2}} v_{1}^{2}+\frac{\partial^{2} F_{2}}{\partial w^{2}} v_{2}^{2}+\frac{\partial^{2} F_{2}}{\partial y^{2}} v_{3}^{2}+2 \frac{\partial^{2} F_{2}}{\partial x \partial w} v_{1} v_{2}+2 \frac{\partial^{2} F_{2}}{\partial x \partial y} v_{1} v_{3}+2 \frac{\partial^{2} F_{2}}{\partial w \partial y} v_{2} v_{3} \\
\frac{\partial^{2} F_{3}}{\partial x^{2}} v_{1}^{2}+\frac{\partial^{2} F_{3}}{\partial w^{2}} v_{2}^{2}+\frac{\partial^{2} F_{3}}{\partial y^{2}} v_{3}^{2}+2 \frac{\partial^{2} F_{3}}{\partial x \partial w} v_{1} v_{2}+2 \frac{\partial^{2} F_{3}}{\partial x \partial y} v_{1} v_{3}+2 \frac{\partial^{2} F_{3}}{\partial w \partial y} v_{2} v_{3}
\end{array}\right)_{E_{2}} \\
& =\frac{2 a_{2} b_{1} c_{1}\left(v_{1}-(m / a)\right)}{\left(b_{1}+\left(\left(r-d_{1}\right) / a_{1}\right)\right)^{2}} \neq 0 .
\end{aligned}
$$

Therefore, by Sotomayor's theorem [43], system (2) exhibits a transcritical bifurcation at $d_{3}=d_{3}^{[\mathrm{TC}]}$ around the axial equilibrium point $E_{2}$.

Theorem 12. System (2) undergoes a transcritical bifurcation around $E_{4}(0, \widehat{w}, \widehat{y})$ if

$$
\begin{aligned}
d_{1}^{[\mathrm{TC}]}= & \frac{r}{1+k \hat{y}}-\frac{a_{2}[a+(1-m) \hat{y}] \hat{y}}{\left(b_{1}+\hat{y}\right)(a+\hat{y})} \\
& \frac{c_{2} a_{3} \widehat{w} \hat{y}}{\left(b_{1}+\widehat{w}\right)(a+\hat{y})}<\frac{c_{2} a_{3} \widehat{w} \hat{y}(a+\hat{y})\left(b_{1}+\widehat{w}\right)}{\left[\left(b_{1}+\widehat{w}\right)(a+\hat{y})\right]^{2}} .
\end{aligned}
$$


Proof. Proof is the same as in Theorem 10.

Theorem 13. System (2) undergoes a transcritical bifurcation around $E_{5}\left(\left(r-d_{1}\right) / a_{1},\left(A / d_{2}\right), 0\right)$ if

$$
d_{3}^{[\mathrm{TC}]}=\frac{c_{1} a_{2}\left(\left(r-d_{1}\right) / a_{1}\right)}{b_{1}+\left(A / d_{2}\right)+\left(\left(r-d_{1}\right) / a_{1}\right)}+\frac{c_{2} a_{3}\left(A / d_{2}\right)}{b_{1}+\left(A / d_{2}\right)+\left(\left(r-d_{1}\right) / a_{1}\right)} .
$$

Proof. Proof is the same as in Theorem 11.

4.3.2. Hopf Bifurcation around $E^{*}\left(x^{*}, w^{*}, y^{*}\right)$. Let us consider $k$ as a bifurcation parameter of system (2) where the characteristic equation at $E^{*}$ is

$$
\lambda^{3}+A_{1}(k) \lambda^{2}+A_{2}(k) \lambda+A_{3}(k)=0 .
$$

Then, Hopf bifurcation theorem is stated as follows.

Theorem 14 (Hopf bifurcation theorem [44]). If $A_{1}(k)$, $A_{2}(k)$, and $A_{3}(k)$ are the smooth functions of $k$ in $N_{\varepsilon}\left(k^{[H]}\right)$, $(\varepsilon>0), k^{[H]} \in \mathbb{R}$ for which the characteristic equation (50) has the following:

(i) A pair of imaginary eigenvalues $\lambda=q_{1}(k) \pm i q_{2}(k)$ with $q_{1}(k)$ and $q_{2}(k) \in \mathbb{R}$ so that they become purely complex at $k=k^{[H]}$ and $\left.\left(d q_{1} / d k\right)\right|_{k=k^{[H]}} \neq 0$

(ii) The other eigenvalue is negative at $k=k^{[H]}$; then, a Hopf bifurcation appears around $E^{*}$ at $k=k^{[H]}$
Theorem 15. If $E^{*}\left(x^{*}, w^{*}, y^{*}\right)$ is locally asymptotically stable, then a Hopf bifurcation is exhibited around $E^{*}\left(x^{*}, w^{*}, y^{*}\right)$ when $k$ passes through its critical value $k^{[H]}$ provided $\quad A_{1}\left(k^{[H]}\right)>0, \quad A_{3}\left(k^{[H]}\right)>0, \quad$ and $A_{1}\left(k^{[H]}\right) A_{2}\left(k^{[H]}\right)=A_{3}\left(k^{[H]}\right) \quad\left(k^{[H]}\right.$ is a positive root of equation $\left.A_{1}(k) A_{2}(k)-A_{3}(k)=0\right)$.

Proof. At $k=k^{[H]}$, we can write equation (50) as

$$
\left(\lambda^{2}+A_{2}\right)\left(\lambda+A_{1}\right)=0 .
$$

The roots of equation (51) are $\lambda_{1}=i \sqrt{A_{2}}, \lambda_{2}=-i \sqrt{A_{2}}$, and $\lambda_{3}=-A_{1}$. Also $A_{1}, A_{2}$, and $A_{3}$ are the smooth functions of $k$. So, the roots of equation (59) have the form $\lambda_{1}=p_{1}(k)+i p_{2}(k), \lambda_{2}=p_{1}(k)-i p_{2}(k)$, and $\lambda_{3}=p_{3}(k)$ where $p_{i}(k)$ are real functions of $k$ in an open neighborhood of $k^{[H]}$ for $i=1,2,3$. Next, we verify the transversality condition:

$$
\left.\frac{\mathrm{d}}{\mathrm{d} k}\left(\operatorname{Re} \lambda_{i}(k)\right)\right|_{k=k^{[H]}} \neq 0, \quad i=1,2 .
$$

Putting $\lambda(k)=p_{1}(k)+i p_{2}(k)$ in (59), we get

$$
p_{1}(k)+i p_{2}(k)^{3}+A_{1}(k) p_{1}(k)+i p_{2}(k)^{2}+A_{2}(k)\left(p_{1}(k)+i p_{2}(k)\right)+A_{3}(k)=0 .
$$

Differentiating both sides with respect to $k$, we have obtain

$$
\begin{aligned}
& 3\left(p_{1}(k)+i p_{2}(k)\right)^{2}\left(\dot{p}_{1}(k)+i \dot{p}_{2}(k)\right)+2 A_{1}(k)\left(p_{1}(k)+i p_{2}(k)\right)\left(\dot{p}_{1}(k)+i \dot{p}_{2}(k)\right) \\
& +\dot{A}_{1}(k)\left(p_{1}(k)+i p_{2}(k)\right)^{2}+A_{2}\left(\dot{p}_{1}(k)+i \dot{p}_{2}(k)\right)+\dot{A}_{2}(k)\left(p_{1}(k)+i p_{2}(k)\right)+\dot{A}_{3}(k)=0 \text {. } \\
& X_{1} \dot{p}_{1}-X_{2} \dot{p}_{2}+X_{3}=0 \\
& X_{2} \dot{p}_{1}+X_{1} \dot{p}_{2}+X_{4}=0
\end{aligned}
$$

Comparing real and imaginary parts from both sides, we

where

$$
\begin{aligned}
& X_{1}=3\left(p_{1}^{2}-p_{2}^{2}\right)+2 A_{1} p_{1}+A_{2} \\
& X_{2}=6 p_{1} p_{2}+2 A_{1} p_{2} \\
& X_{3}=\dot{A}_{1}\left(p_{1}^{2}-p_{2}^{2}\right)+\dot{A}_{2} p_{1}+\dot{A}_{3} \neq \dot{A}_{1}\left(p_{1}^{2}-p_{2}^{2}\right)+\dot{A}_{2} p_{1}+A_{1} \dot{A}_{2}+A_{2} \dot{A}_{1},
\end{aligned}
$$

[since $A_{3} \neq A_{1} A_{2}$ in a deleted neighbour hood of $k^{[H]}$, 
and $X_{4}=2 \dot{A}_{1} p_{1} p_{2}+\dot{A}_{2} p_{2}$.

Multiplying (55) by $X_{1}$ and (56) by $X_{2}$ and then adding, we get

$$
\left(X_{1}^{2}+X_{2}^{2}\right) \dot{p}_{1}+X_{1} X_{3}+X_{2} X_{4}=0 \Longrightarrow \dot{p}_{1}=-\left\{\frac{X_{1} X_{3}+X_{2} X_{4}}{X_{1}^{2}+X_{2}^{2}}\right\} .
$$

At $k=k^{[H]}$,

Case 1: $\quad p_{1}=0, \quad p_{2}=\sqrt{A_{2}}$. Then, $\quad X_{1}=-2 A_{2}$, $X_{2}=2 A_{1} \sqrt{A_{2}}, \quad X_{3} \neq A_{1} \dot{A}_{2}, \quad$ and $\quad X_{4}=\dot{A}_{2} \sqrt{A_{2}}$. $\therefore X_{1} X_{3}+X_{2} X_{4} \neq 0$.

Case 2: $p_{1}=0, \quad p_{2}=-\sqrt{A_{2}}$. Then, $X_{1}=-2 A_{2}$, $X_{2}=-2 A_{1} \sqrt{A_{2}}, \quad X_{3} \neq A_{1} \dot{A}_{2}, \quad$ and $\quad X_{4}=-\dot{A}_{2} \sqrt{A_{2}}$. $\therefore X_{1} X_{3}+X_{2} X_{4} \neq 0$

Also, $\lambda_{3}=-A_{1}\left(k^{[H]}\right)<0$. 14.

Hence, this theorem is proved by virtue of Theorem

4.3.3. Hopf Bifurcation around $E_{3}(\bar{x}, 0, \bar{y})$. Let us consider $k$ as a bifurcation parameter of system (2) where the characteristic equation of $E_{3}$ is

$$
\lambda^{3}+B_{1}(k) \lambda^{2}+B_{2}(k) \lambda+B_{3}(k)=0,
$$

and then Hopf bifurcation theorem is stated as follows.

Theorem 16 (Hopf bifurcation theorem [44]). If $B_{1}(k)$, $B_{2}(k)$, and $B_{3}(k)$ are the smooth functions of $k$ in $N_{\varepsilon}\left(k^{*}\right)$, $(\varepsilon>0), k^{*} \in \mathbb{R}$ for which the characteristic equation (59) has the following: (i) A pair of imaginary eigenvalues $\lambda=p_{1}^{\prime}(k) \pm i p_{2}^{\prime}(k)$ with $p_{1}^{\prime}(k)$ and $p_{2}^{\prime}(k) \in \mathbb{R}$ so that they become purely imaginary at $k=k^{*}$ and $\left.\left(d p_{1}^{\prime} / d k\right)\right|_{k=k^{*}} \neq 0$

(ii) The other eigenvalue is negative at $k=k^{*}$; then a Hopf bifurcation occurs around $E_{3}(\bar{x}, 0, \bar{y})$ at $k=k^{*}$

Theorem 17. If $E_{3}(\bar{x}, 0, \bar{y})$ is locally asymptotically stable, then a Hopf bifurcation appears around subsidy-free equilibrium $E_{3}(\bar{x}, 0, \bar{y})$ when $k$ passes through its critical value $k^{*}$ provided $\quad B_{1}\left(k^{*}\right)>0, \quad B_{3}\left(k^{*}\right)>0, \quad$ and $B_{1}\left(k^{*}\right) B_{2}\left(k^{*}\right)=B_{3}\left(k^{*}\right)$ ( $k^{*}$ is a positive root of equation $\left.B_{1}(k) B_{2}(k)-B_{3}(k)=0\right)$.

Proof. Proof is the same as in Theorem 15.

\section{Delayed Dynamical System}

In biological point of view, many processes, both natural and man-made, include time-delay. The study of delay factor makes our system much more realistic than non-delayed system. Also, a delay differential equation reveals much more complicated dynamics than an ordinary differential equation (for details, see [10-13, 23, 45-49]).

In reality, after sensing the vocal cue, individuals of prey species take some time for assessing the predation risk. So, the effect of fear (felt by prey) of predator does not respond spontaneously on the birth rate of prey population; some time lag must be needed. In view of this fact, the predatorprey-subsidy interactions (2) can be modified as follows:

$$
\begin{aligned}
& \frac{\mathrm{d} x}{\mathrm{~d} t}=\frac{r x}{1+k y(t-\tau)}-d_{1} x-a_{1} x^{2}-\frac{a_{2}(1-(m y /(a+y))) x y}{b_{1}+w+(1-(m y /(a+y))) x} \\
& \frac{\mathrm{d} w}{\mathrm{~d} t}=A-d_{2} w-\frac{a_{3} w y}{b_{1}+w+(1-(m y /(a+y))) x}, \\
& \frac{\mathrm{d} y}{\mathrm{~d} t}=\frac{c_{1} a_{2}(1-(m y /(a+y))) x y}{b_{1}+w+(1-(m y /(a+y))) x}+\frac{c_{2} a_{3} w y}{b_{1}+w+(1-(m y /(a+y))) x}-d_{3} y .
\end{aligned}
$$

The initial conditions are assumed as $(i=1,2,3)$

$$
\begin{gathered}
\psi_{i}(\phi)>0, \phi \in[-\tau, 0], \quad \text { where } x(\phi)=\psi_{1}(\phi), w(\phi)=\psi_{2}(\phi), y(\phi)=\psi_{3}(\phi), \\
\text { For biological feasibility: } \psi_{1}(0)>0, \psi_{2}(0)>0, \psi_{3}(0)>0 .
\end{gathered}
$$

Let us linearize (60) using the following transformations:

$$
\begin{aligned}
X & =x-\bar{x}, \\
W & =w-0, \\
Y & =y-\bar{y} .
\end{aligned}
$$

It leads to

$$
\frac{\mathrm{d} U}{\mathrm{~d} t}=B_{1}^{\prime} U(t)+B_{2}^{\prime} U(t-\tau),
$$

where $U=[X, W, Y]^{T}$, 


$$
\begin{aligned}
& B_{1}^{\prime}=\left[\begin{array}{lll}
b_{11} & b_{12} & b_{13}^{\prime} \\
b_{21} & b_{22} & b_{23} \\
b_{31} & b_{32} & b_{33}
\end{array}\right], \\
& B_{2}^{\prime}=\left[\begin{array}{ccc}
0 & 0 & b_{13}^{\prime \prime} \\
0 & 0 & 0 \\
0 & 0 & 0
\end{array}\right] \text {, } \\
& b_{11}=\bar{x}\left\{-a_{1}+\frac{a_{2} \bar{y}(a+(1-m) \bar{y})^{2}}{\left\{b_{1}(a+\bar{y})+[a+(1-m) \bar{y}] \bar{x}\right\}^{2}}\right\}, \\
& b_{12}=\frac{a_{2} \overline{x y}[a+(1-m) \bar{y}](a+\bar{y})}{\left\{b_{1}(a+\bar{y})+[a+(1-m) \bar{y}] \bar{x}\right\}^{2}}, \\
& b_{13}^{\prime}=\bar{x}\left\{-\frac{a_{2}[a+2(1-m) \bar{y}]}{b_{1}(a+\bar{y})+[a+(1-m) \bar{y}] \bar{x}}+\frac{a_{2} \bar{y}[a+(1-m) \bar{y}]\left[b_{1}+(1-m) \bar{x}\right]}{\left\{b_{1}(a+\bar{y})+[a+(1-m) \bar{y}] \bar{x}\right\}^{2}}\right\}, \\
& b_{21}=0 \text {, } \\
& b_{22}=-d_{2}-\frac{a_{3} \bar{y}}{b_{1}+(1-(m \bar{y} /(a+\bar{y}))) \bar{x}}, \\
& b_{23}=0 \text {, } \\
& b_{31}=\bar{y}\left\{\frac{c_{1} a_{2}[a+(1-m) \bar{y}]}{b_{1}(a+\bar{y})+[a+(1-m) \bar{y}] \bar{x}}-\frac{c_{1} a_{2}[a+(1-m) \bar{y}]^{2} \bar{x}}{\left\{b_{1}(a+\bar{y})+[a+(1-m) \bar{y}] \bar{x}\right\}^{2}}\right\}, \\
& b_{32}=\frac{c_{2} a_{3} \bar{y}(a+\bar{y})}{b_{1}\left(a+y^{*}\right)+[a+(1-m) \bar{y}] \bar{x}}, \\
& b_{33}=\bar{y}\left\{\frac{c_{1} a_{2}(1-m) \bar{x}}{b_{1}(a+\bar{y})+[a+(1-m) \bar{y}] \bar{x}}-\frac{c_{1} a_{2}[a+(1-m) \bar{y}] \bar{x}\left[b_{1}+(1-m) \bar{x}\right]}{\left\{b_{1}(a+\bar{y})+[a+(1-m) \bar{y}] \bar{x}\right\}^{2}}\right\} \text {, } \\
& b_{13}^{\prime \prime}=-\frac{r k \bar{x}}{(1+k \bar{y})^{2}} \text {. }
\end{aligned}
$$

The characteristic equation corresponding to (62) is

$$
\lambda^{3}+L_{1} \lambda^{2}+L_{2} \lambda+L_{3}+\left(M_{1} \lambda+M_{2}\right) e^{-\lambda \tau}=0,
$$

where

$$
\begin{aligned}
L_{1} & =-\left(b_{11}+b_{22}+b_{33}\right), \\
L_{2} & =b_{22} b_{33}+b_{11} b_{33}-b_{13}^{\prime} b_{31}+b_{11} b_{22}, \\
L_{3} & =-\left[b_{11} b_{22} b_{33}-b_{13}^{\prime} b_{22} b_{31}\right], \\
M_{1} & =-b_{13}^{\prime \prime} b_{31} \\
M_{2} & =b_{13}^{\prime \prime} b_{31} b_{22} .
\end{aligned}
$$

If $\tau \neq 0, E_{3}$ of system (60) is LAS provided equation (64) has no purely imaginary roots and it is also LAS for $\tau=0$. Further, it has been shown that stability nature of $E_{3}$ switches at $\tau=\tau^{\prime *}$. Already, it has been derived that $E_{3}$ is LAS provided $B_{1}>0, B_{3}>0$, and $B_{1} B_{2}>B_{3}$ for $\tau=0$ (nondelayed system). Let us discuss if the real part of the roots of equation (64) gradually increases to reach zero and eventually turns to a positive value when $\tau$ increases.

Substituting $\lambda=q_{1}^{\prime}+i q_{2}^{\prime}$ in equation (64), we have

$$
\begin{aligned}
& \left(q_{1}^{\prime}+i q_{2}^{\prime}\right)^{3}+L_{1}\left(q_{1}^{\prime}+i q_{2}^{\prime}\right)^{2}+L_{2}\left(q_{1}^{\prime}+i q_{2}^{\prime}\right)+L_{3} \\
& \quad+\left(M_{1}\left(q_{1}^{\prime}+i q_{2}^{\prime}\right)+M_{2}\right) e^{-q_{1}^{\prime} \tau}\left(\cos \left(q_{2}^{\prime} \tau\right)-i \sin \left(q_{2}^{\prime} \tau\right)\right)=0 .
\end{aligned}
$$


Equating respective real and complex parts from both sides, we obtain

$$
\begin{array}{r}
q_{1}^{\prime 3}-3 q_{1}^{\prime} q_{2}^{\prime 2}+L_{1}\left(q_{1}^{\prime 2}-q_{2}^{\prime 2}\right)+L_{2} q_{1}^{\prime}+L_{3}+M_{1} q_{1}^{\prime} e^{-q_{1}^{\prime} \tau} \cos \left(q_{2}^{\prime} \tau\right)+M_{2} e^{-q_{1}^{\prime} \tau} \cos \left(q_{2}^{\prime} \tau\right)+M_{1} q_{2}^{\prime} e^{-q_{1}^{\prime} \tau} \sin \left(q_{2}^{\prime} \tau\right)=0 \\
3 q_{1}^{\prime 2} q_{2}^{\prime}-q_{2}^{\prime 3}+2 L_{1} q_{1}^{\prime} q_{2}^{\prime}+L_{2} q_{2}^{\prime}+M_{1} q_{2}^{\prime} e^{-q_{1}^{\prime} \tau} \cos \left(q_{2}^{\prime} \tau\right)-M_{1} q_{1}^{\prime} e^{-q_{1}^{\prime} \tau} \sin \left(q_{2}^{\prime} \tau\right)-M_{2} e^{-q_{1}^{\prime} \tau} \sin \left(q_{2}^{\prime} \tau\right)=0
\end{array}
$$

Now, let us examine whether equation (64) has purely imaginary roots or not. For this purpose, let us take $q_{1}^{\prime}=0$. Then, equations (67) and (68) become

$$
\begin{aligned}
& M_{1} q_{2}^{\prime} \sin \left(q_{2}^{\prime} \tau\right)+M_{2} \cos \left(q_{2}^{\prime} \tau\right)=L_{1} q_{2}^{\prime 2}-L_{3}, \\
& M_{1} q_{2}^{\prime} \cos \left(q_{2}^{\prime} \tau\right)-M_{2} \sin \left(q_{2}^{\prime} \tau\right)=q_{2}^{\prime 3}-L_{2} q_{2}^{\prime} .
\end{aligned}
$$
we get

Eliminating $\tau$ from (69) and (70) (squaring and adding),

$$
q_{2}^{\prime 6}+q_{2}^{\prime}\left(L_{1}^{2}-2 L_{2}\right)+q_{2}^{\prime 2}\left(L_{2}^{2}-2 L_{1} L_{3}-M_{1}^{2}\right)+\left(L_{3}^{2}-M_{2}^{2}\right)=0 .
$$

Putting $q_{2}^{\prime 2}=\beta$, we have

$$
L(\beta) \equiv \beta^{3}+\left(L_{1}^{2}-2 L_{2}\right) \beta^{2}+\left(L_{2}^{2}-2 L_{1} L_{3}-M_{1}^{2}\right) \beta+\left(L_{3}^{2}-M_{2}^{2}\right)=0 .
$$

This is a cubic equation of $\beta$. It is noticed that $L(\infty)=\infty$. So, equation (72) has exactly one positive real root if $L(0)<0$, i.e., if $L_{3}^{2}<M_{2}^{2}$.
Let $\beta=\beta_{+}$be a positive root of (77); then, $q_{2}^{\prime}=\sqrt{\beta_{+}}$.

Lemma 1 (see [50]). Consider the exponential polynomial:

$$
\begin{aligned}
P(\lambda) \equiv & P\left(\lambda, \tau_{1}, \tau_{2}, \ldots, \tau_{m}\right) \equiv \lambda^{n}+p_{1}^{(0)} \lambda^{n-1}+\cdots+p_{n-1}^{(0)} \lambda+p_{n}^{(0)}+\left[p_{1}^{(1)} \lambda^{n-1}+\cdots+p_{n-1}^{(1)} \lambda+p_{n}^{(1)}\right] e^{-\lambda \tau_{1}}+\cdots \\
& +\left[p_{1}^{(m)} \lambda^{n-1}+\cdots+p_{n-1}^{(m)} \lambda+p_{n}^{(m)}\right] e^{-\lambda \tau_{m}}
\end{aligned}
$$

where $\tau_{i} \geq 0(i=1,2, \ldots, m)$ and $p_{j}^{(i)}(i=0,1, \ldots, m ; j=$ $1,2, \ldots, n)$ are constants. As $\left(\tau_{1}, \tau_{2}, \ldots, \tau_{m}\right)$ vary, the sum of the orders of zero of $P(\lambda)$ in the open half plane can change only if a zero appears on or crosses the imaginary axis.
Theorem 18. Suppose $E_{3}$ exists and is locally asymptotically stable for system (2) when $\tau=0$. If $L_{3}^{2}<M_{2}^{2}$, then there exists a critical value $\tau^{\prime *}$ such that $E_{3}$ of system (60) is LAS when $\tau \in\left[0, \tau^{\prime *}\right)$ and unstable when $\tau>\tau^{\prime *}$, where

Now, let us discuss the existence of Hopf bifurcation around $E_{3}$ with $\tau$ as a bifurcation parameter.

$$
\tau_{+}^{\prime(j)}=\frac{\cos ^{-1}\left(\left(M_{2}\left(L_{1} \beta_{+}-L_{3}\right)+M_{1}\left(\beta_{+}^{2}-L_{2} \beta_{+}\right)\right) /\left(M_{2}^{2}+M_{1}^{2} \beta_{+}\right)\right)}{\sqrt{\beta_{+}}}+\frac{2 \pi j}{\sqrt{\beta_{+}}}, \quad j=0,1,2,3, \ldots
$$

and ${\tau^{\prime *}}^{*}=\tau_{+}^{\prime(0)}$ (minimum value). Also, system (60) exhibits Hopf bifurcation around $E_{3}$ at $\tau=\tau^{\prime *}$ provided $K^{\prime} M^{\prime}-L^{\prime} N^{\prime} \neq 0$, where 


$$
\begin{aligned}
K^{\prime} & =\left\{-3 \beta_{+}+L_{2}+M_{1} \cos \left(\sqrt{\beta_{+}} \tau^{\prime *}\right)-M_{2} \tau^{\prime *} \cos \left(\sqrt{\beta_{+}} \tau^{\prime *}\right)-M_{1} \sqrt{\beta_{+}} \tau^{\prime *} \sin \left(\sqrt{\beta_{+}} \tau^{\prime *}\right)\right\}, \\
L^{\prime} & =\left\{-2 L_{1} \sqrt{\beta_{+}}+M_{1} \sin \left(\sqrt{\beta_{+}} \tau^{\prime *}\right)-M_{2} \tau^{\prime *} \sin \left(\sqrt{\beta_{+}} \tau^{\prime *}\right)+M_{1} \sqrt{\beta_{+}} \tau^{\prime *} \cos \left(\sqrt{\beta_{+}} \tau^{\prime *}\right)\right\}, \\
M^{\prime} & =\left\{M_{2} \sqrt{\beta_{+}} \sin \left(\sqrt{\beta_{+}} \tau^{\prime *}\right)-M_{1} \beta_{+} \cos \left(\sqrt{\beta_{+}} \tau^{\prime *}\right)\right\}, \\
N^{\prime} & =\left\{M_{2} \sqrt{\beta_{+}} \cos \left(\sqrt{\beta_{+}} \tau^{\prime *}\right)+M_{1} \beta_{+} \sin \left(\sqrt{\beta_{+}} \tau^{\prime *}\right)\right\} .
\end{aligned}
$$

Proof. If $L_{3}^{2}<M_{2}^{2}$, then (72) has exactly one positive $\operatorname{root} \beta_{+}$, i.e., from (69) and (70), $\tau_{+}{ }^{(j)}, j=0,1,2, \ldots$, are obtained as functions of $\beta_{+}$:

$$
\begin{aligned}
& \tau_{+}^{\prime(j)}=\frac{\cos ^{-1}\left(\left(M_{2}\left(L_{1} \beta_{+}-L_{3}\right)+M_{1}\left(\beta_{+}^{2}-L_{2} \beta_{+}\right)\right) /\left(M_{2}^{2}+M_{1}^{2} \beta_{+}\right)\right)}{\sqrt{\beta_{+}}}+\frac{2 \pi j}{\sqrt{\beta_{+}}}, \quad j=0,1,2,3, \ldots \\
& \text { cally asymptotically stable, the stability be- } \\
& \text { will remain unaltered for } \tau<\tau^{\prime *} \text { (using Butler's }
\end{aligned}
$$

Lemma [51]).

To check the transversality condition, $[(\mathrm{d} / \mathrm{d} \tau) \operatorname{Re} \lambda(\tau)]_{\tau=\tau^{\prime *}} \neq 0$, let us differentiate (67) and (68) with respect to $\tau$ and set $q_{1}^{\prime}=0$ and $\tau=\tau^{\prime *}$. The following equations are obtained:

$$
-L^{\prime}\left[\frac{\mathrm{d}}{\mathrm{d} \tau}[\operatorname{Re}\{\lambda(\tau)\}]\right]_{\tau=\tau^{\prime *}}+K^{\prime}\left[\frac{\mathrm{d}}{\mathrm{d} \tau}[\operatorname{Im}\{\lambda(\tau)\}]\right]_{\tau=\tau^{\prime *}}=N^{\prime}
$$

where

$$
\begin{aligned}
K^{\prime} & =\left\{-3 \beta_{+}+L_{2}+M_{1} \cos \left(\sqrt{\beta_{+}} \tau^{\prime *}\right)-M_{2} \tau^{\prime *} \cos \left(\sqrt{\beta_{+}} \tau^{\prime *}\right)-M_{1} \sqrt{\beta_{+}} \tau^{\prime *} \sin \left(\sqrt{\beta_{+}} \tau^{\prime *}\right)\right\}, \\
L^{\prime} & =\left\{-2 L_{1} \sqrt{\beta_{+}}+M_{1} \sin \left(\sqrt{\beta_{+}} \tau^{\prime *}\right)-M_{2} \tau^{\prime *} \sin \left(\sqrt{\beta_{+}} \tau^{\prime *}\right)+M_{1} \sqrt{\beta_{+}} \tau^{\prime *} \cos \left(\sqrt{\beta_{+}} \tau^{\prime *}\right)\right\}, \\
M^{\prime} & =\left\{M_{2} \sqrt{\beta_{+}} \sin \left(\sqrt{\beta_{+}} \tau^{\prime *}\right)-M_{1} \beta_{+} \cos \left(\sqrt{\beta_{+}} \tau^{\prime *}\right)\right\}, \\
N^{\prime} & =\left\{M_{2} \sqrt{\beta_{+}} \cos \left(\sqrt{\beta_{+}} \tau^{\prime *}\right)+M_{1} \beta_{+} \sin \left(\sqrt{\beta_{+}} \tau^{\prime *}\right)\right\} .
\end{aligned}
$$

Solving (77) and (78),

$$
\left[\frac{\mathrm{d}[\operatorname{Re}\{\lambda(\tau)\}]}{\mathrm{d} \tau}\right]_{\tau=\tau^{\prime *}}=\left[\frac{K^{\prime} M^{\prime}-L^{\prime} N^{\prime}}{K^{\prime 2}+L^{\prime 2}}\right] .
$$

Now, we have $\mathrm{d}[\operatorname{Re}\{\lambda(\tau)\}] / \mathrm{d} \tau_{\tau=\tau^{\prime}{ }^{*}} \neq 0, \quad$ if $K^{\prime} M^{\prime}-L^{\prime} N^{\prime} \neq 0$. Hence, the transversality condition is satisfied and a Hopf bifurcation occurs around $E^{*}$ when $\tau$ passes through its critical value $\tau^{\prime *}$.

Now, linearize system (60) using the transformations $X=x-x^{*}, W=w-w^{*}$, and $Y=y-y^{*}$ :

$$
\frac{\mathrm{d} U}{\mathrm{~d} t}=A_{1}^{\prime} U(t)+A_{2}^{\prime} U(t-\tau)
$$


where $U=[X, W, Y]^{T}$,

$$
\begin{aligned}
& A_{1}^{\prime}=\left[\begin{array}{lll}
a_{11} & a_{12} & a_{13}^{\prime} \\
a_{21} & a_{22} & a_{23} \\
a_{31} & a_{32} & a_{33}
\end{array}\right], \\
& A_{2}^{\prime}=\left[\begin{array}{lll}
0 & 0 & a_{13}^{\prime \prime} \\
0 & 0 & 0 \\
0 & 0 & 0
\end{array}\right] \text {, } \\
& a_{11}=x^{*}\left[-a_{1}+\frac{a_{2}\left(a+(1-m) y^{*}\right)^{2} y^{*}}{\left[\left(b_{1}+w^{*}\right)\left(a+y^{*}\right)+\left(a+(1-m) y^{*}\right) x^{*}\right]^{2}}\right] \text {, } \\
& a_{12}=\frac{a_{2}\left(a+(1-m) y^{*}\right)\left(a+y^{*}\right) x^{*} y^{*}}{\left[\left(b_{1}+w^{*}\right)\left(a+y^{*}\right)+\left(a+(1-m) y^{*}\right) x^{*}\right]^{2}}, \\
& a_{13}^{\prime}=\left[-\frac{a_{2}\left(a+2(1-m) y^{*}\right) x^{*}}{\left(b_{1}+w^{*}\right)\left(a+y^{*}\right)+\left(a+(1-m) y^{*}\right) x^{*}}+\frac{a_{2}\left(a+(1-m) y^{*}\right) x^{*} y^{*}\left(b_{1}+w^{*}+(1-m) x^{*}\right)}{\left[\left(b_{1}+w^{*}\right)\left(a+y^{*}\right)+\left(a+(1-m) y^{*}\right) x^{*}\right]^{2}}\right] \text {, } \\
& a_{21}=\frac{a_{3}\left(a+y^{*}\right)\left(a+(1-m) y^{*}\right) w^{*} y^{*}}{\left[\left(b_{1}+w^{*}\right)\left(a+y^{*}\right)+\left(a+(1-m) y^{*}\right) x^{*}\right]^{2}}, \\
& a_{22}=-d_{2}-\frac{a_{3}\left(a+y^{*}\right) y^{*}}{\left(b_{1}+w^{*}\right)\left(a+y^{*}\right)+\left(a+(1-m) y^{*}\right) x^{*}}+\frac{a_{3}\left(a+y^{*}\right)^{2} w^{*} y^{*}}{\left[\left(b_{1}+w^{*}\right)\left(a+y^{*}\right)+\left(a+(1-m) y^{*}\right) x^{*}\right]^{2}}, \\
& a_{23}=-\frac{a_{3}\left(a+2 y^{*}\right) w^{*}}{\left(b_{1}+w^{*}\right)\left(a+y^{*}\right)+\left(a+(1-m) y^{*}\right) x^{*}}+\frac{a_{3}\left(a+y^{*}\right)\left(b_{1}+w^{*}+(1-m) x^{*}\right)}{\left[\left(b_{1}+w^{*}\right)\left(a+y^{*}\right)+\left(a+(1-m) y^{*}\right) x^{*}\right]^{2}}, \\
& a_{31}=\frac{c_{1} a_{2}\left(a+(1-m) y^{*}\right) y^{*}}{\left(b_{1}+w^{*}\right)\left(a+y^{*}\right)+\left(a+(1-m) y^{*}\right) x^{*}}-\frac{c_{1} a_{2}\left(a+(1-m) y^{*}\right)^{2} x^{*} y^{*}}{\left[\left(b_{1}+w^{*}\right)\left(a+y^{*}\right)+\left(a+(1-m) y^{*}\right) x^{*}\right]^{2}} \\
& -\frac{c_{2} a_{3} w^{*} y^{*}\left(a+y^{*}\right)\left(a+(1-m) y^{*}\right)}{\left[\left(b_{1}+w^{*}\right)\left(a+y^{*}\right)+\left(a+(1-m) y^{*}\right) x^{*}\right]^{2}} \\
& a_{32}=-\frac{c_{1} a_{2}\left(a+(1-m) y^{*}\right) x^{*} y^{*}\left(a+y^{*}\right)}{\left[\left(b_{1}+w^{*}\right)\left(a+y^{*}\right)+\left(a+(1-m) y^{*}\right) x^{*}\right]^{2}}+\frac{c_{2} a_{3}\left(a+y^{*}\right) y^{*}}{\left(b_{1}+w^{*}\right)\left(a+y^{*}\right)+\left(a+(1-m) y^{*}\right) x^{*}} \\
& -\frac{c_{2} a_{3} w^{*} y^{*}\left(a+y^{*}\right)^{2}}{\left[\left(b_{1}+w^{*}\right)\left(a+y^{*}\right)+\left(a+(1-m) y^{*}\right) x^{*}\right]^{2}} \\
& a_{33}=\frac{c_{1} a_{2}(1-m) x^{*} y^{*}+c_{2} a_{3} w^{*} y^{*}}{\left(b_{1}+w^{*}\right)\left(a+y^{*}\right)+\left(a+(1-m) y^{*}\right) x^{*}}-\frac{\left[c_{1} a_{2}\left(a+(1-m) y^{*}\right) x^{*} y^{*}+c_{2} a_{3} w^{*} y^{*}\left(a+y^{*}\right)\right]\left(b_{1}+w^{*}+(1-m) x^{*}\right)}{\left[\left(b_{1}+w^{*}\right)\left(a+y^{*}\right)+\left(a+(1-m) y^{*}\right) x^{*}\right]^{2}}, \\
& a_{13}^{\prime \prime}=-\frac{r k x^{*}}{\left(1+k y^{*}\right)^{2}}
\end{aligned}
$$


The characteristic equation corresponding to (81) is

$$
\lambda^{3}+R_{1} \lambda^{2}+R_{2} \lambda+R_{3}+\left(S_{1} \lambda+S_{2}\right) e^{-\lambda \tau}=0
$$

where

$$
\begin{aligned}
& R_{1}=-\left(a_{11}+a_{22}+a_{33}\right), \\
& R_{2}=a_{22} a_{33}-a_{23} a_{32}+a_{11} a_{33}-a_{13}^{\prime} a_{31}+a_{11} a_{22}-a_{12} a_{21}, \\
& R_{3}=-\left[a_{11}\left(a_{22} a_{33}-a_{23} a_{32}\right)+a_{12}\left(a_{23} a_{31}-a_{21} a_{33}\right)+a_{13}^{\prime}\left(a_{21} a_{32}-a_{22} a_{31}\right)\right], \\
& S_{1}=-a_{13}^{\prime \prime} a_{31}, \\
& S_{2}=-a_{13}^{\prime \prime}\left(a_{21} a_{32}-a_{31} a_{22}\right) .
\end{aligned}
$$

If $\tau \neq 0, E^{*}$ of system (60) is LAS provided equation (83) has no purely imaginary roots and it is LAS for $\tau=0$. Furthermore, it has to be noted that changes of stability occur at $\tau=\tau^{*}$. Already, it has been discussed that $E^{*}$ is LAS when $\tau=0$ provided $A_{1}>0, A_{3}>0$, and $A_{1} A_{2}>A_{3}$. Here, equation (83) is a transcendental equation, so it contains infinitely many eigenvalues. In this situation, we cannot apply the Routh-Hurwitz criteria to determine the stability of system (60). To understand the stability behaviour, our necessity is to check the sign of the real parts of the eigenvalues of equation (83).

Now, putting $\lambda=q_{1}+i q_{2}$ in equation (83), we have

$$
\left(q_{1}+i q_{2}\right)^{3}+R_{1}\left(q_{1}+i q_{2}\right)^{2}+R_{2}\left(q_{1}+i q_{2}\right)+R_{3}+\left(S_{1}\left(q_{1}+i q_{2}\right)+S_{2}\right) e^{-q_{1} \tau}\left(\cos \left(q_{2} \tau\right)-i \sin \left(q_{2} \tau\right)\right)=0
$$

Equating respective real and complex parts from both sides, we get

$$
\begin{array}{r}
q_{1}^{3}-3 q_{1} q_{2}^{2}+R_{1}\left(q_{1}^{2}-q_{2}^{2}\right)+R_{2} q_{1}+R_{3}+S_{1} q_{1} e^{-q_{1} \tau} \cos \left(q_{2} \tau\right)+S_{2} e^{-q_{1} \tau} \cos \left(q_{2} \tau\right)+S_{1} q_{2} e^{-q_{1} \tau} \sin \left(q_{2} \tau\right)=0, \\
\begin{array}{r}
3 q_{1}^{2} q_{2}-q_{2}^{3}+2 R_{1} q_{1} q_{2}+R_{2} q_{2}+S_{1} q_{2} e^{-q_{1} \tau} \cos \left(q_{2} \tau\right)-S_{1} q_{1} e^{-q_{1} \tau} \sin \left(q_{2} \tau\right)-S_{2} e^{-q_{1} \tau} \sin \left(q_{2} \tau\right)=0 . \\
R^{\prime}(\sigma) \equiv \sigma^{3}+\left(R_{1}^{2}-2 R_{2}\right) \sigma^{2}+\left(R_{2}^{2}-2 R_{1} R_{3}-S_{1}^{2}\right) \sigma+\left(R_{3}^{2}-S_{2}^{2}\right)=0 .
\end{array}
\end{array}
$$

To check whether (83) has purely imaginary roots or not, set $q_{1}=0$; then, (86) and (87) become

$$
\begin{aligned}
& S_{1} q_{2} \sin \left(q_{2} \tau\right)+S_{2} \cos \left(q_{2} \tau\right)=R_{1} q_{2}^{2}-R_{3}, \\
& S_{1} q_{2} \cos \left(q_{2} \tau\right)-S_{2} \sin \left(q_{2} \tau\right)=q_{2}^{3}-R_{2} q_{2} .
\end{aligned}
$$

Eliminating $\tau$ from (88) and (89) (squaring and adding), we get

$$
q_{2}^{6}+q_{2}^{4}\left(R_{1}^{2}-2 R_{2}\right)+q_{2}^{2}\left(R_{2}^{2}-2 R_{1} R_{3}-S_{1}^{2}\right)+\left(R_{3}^{2}-S_{2}^{2}\right)=0 .
$$

Putting $q_{2}^{2}=\sigma$, we have
This is a cubic equation of $\sigma$. It is noted that $R^{\prime}(\infty)=\infty$. So, equation (91) has exactly one positive root if $R^{\prime}(0)<0$, i.e., if $R_{3}^{2}<S_{2}^{2}$.

Let $\sigma=\sigma_{+}$be a positive root of (91); then, $q_{2}=\sqrt{\sigma_{+}}$.

Let us study the existence of Hopf bifurcation around $E^{*}$ with $\tau$ as bifurcation parameter.

Theorem 19. Suppose $E^{*}$ exists and is locally asymptotically stable for system (2) when $\tau=0$. If $R_{3}^{2}<S_{2}^{2}$, then there exists a critical value $\tau^{*}$ such that $E^{*}$ of system (60) is LAS when $\tau \in\left[0, \tau^{*}\right)$ and unstable when $\tau>\tau^{*}$, where

$$
\tau_{+}^{(j)}=\frac{\cos ^{-1}\left(\left(S_{2}\left(R_{1} \sigma_{+}-R_{3}\right)+S_{1}\left(\sigma_{+}^{2}-R_{2} \sigma_{+}\right)\right) /\left(S_{2}^{2}+S_{1}^{2} \sigma_{+}\right)\right)}{\sqrt{\sigma_{+}}}+\frac{2 \pi j}{\sqrt{\sigma_{+}}}, \quad j=0,1,2,3, \ldots
$$

and $\tau^{*}=\tau_{+}^{(0)}$ (minimum value). Also, a supercritical Hopf bifurcation is exhibited around $E^{*}$ at $\tau=\tau^{*}$ provided $K^{\prime \prime} M^{\prime \prime}-L^{\prime \prime} N^{\prime \prime} \neq 0$, where 


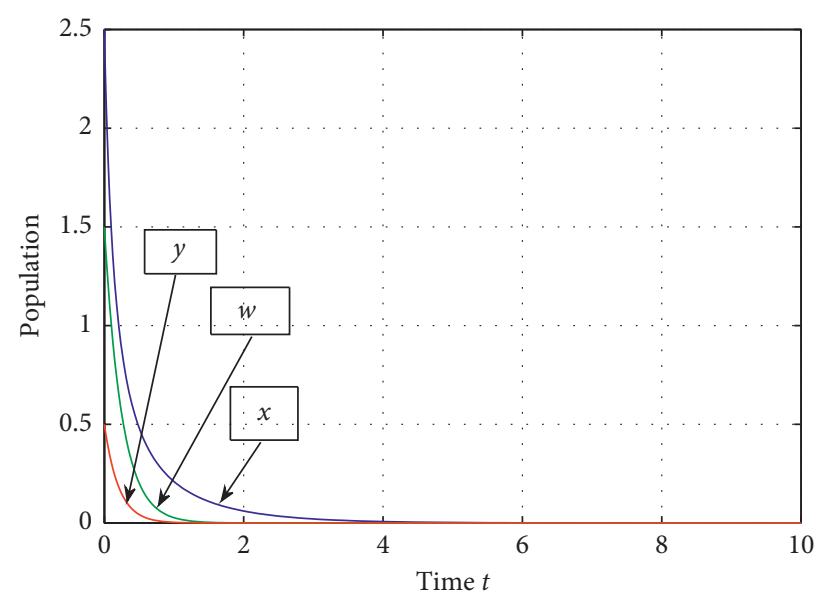

Figure 1: Stable behaviour of $E_{0}(0,0,0)$ with respect to time $t$ corresponding to the data set $\left\{r=5.5, d_{1}=6.5, d_{2}=4, k=0.2, d_{3}=5, a_{1}=2\right.$, $\left.a_{2}=0.3, a_{3}=0.25, b_{1}=1.5, c_{1}=0.7, c_{2}=0.5, A=0, a=1.2, m=0.01\right\}$.

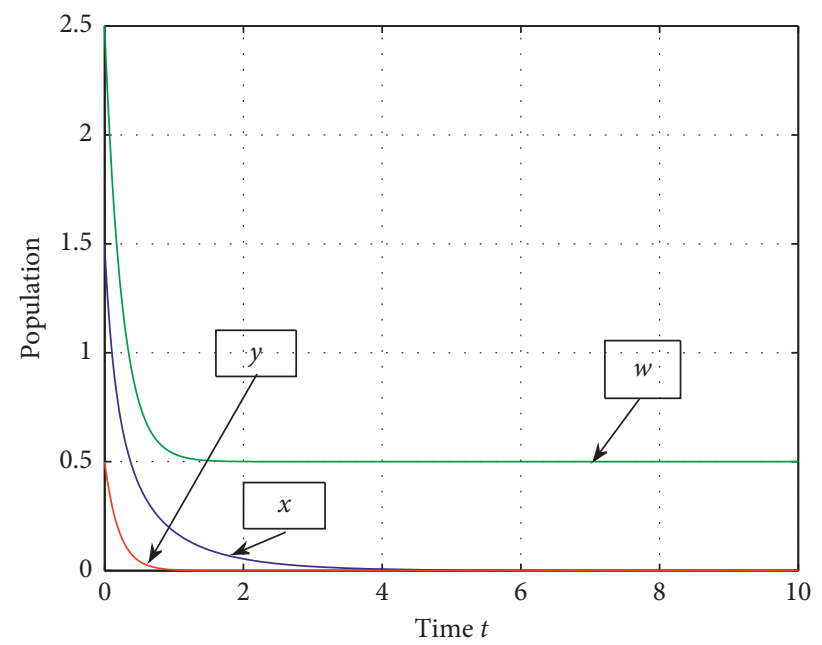

Figure 2: Stable behaviour of $E_{1}(0,0.5,0)$ with respect to time $t$ taking the parameters as $\left\{r=5.5, d_{1}=6.5, d_{2}=4, k=0.2, d_{3}=5, a_{1}=2\right.$, $\left.a_{2}=0.3, a_{3}=0.25, b_{1}=1.5, c_{1}=0.7, c_{2}=0.5, A=2, a=1.2, m=0.01\right\}$.

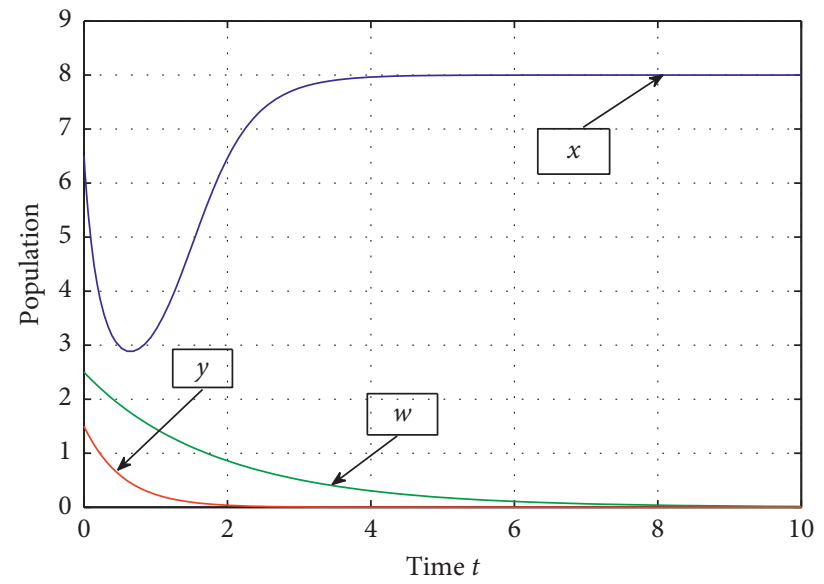

FIgURE 3: Stable nature of $E_{2}(8,0,0)$ with respect to time $t$ regarding the parameters as $\left\{r=5.5, d_{1}=1.5, d_{2}=0.52, k=1.9, d_{3}=2, a_{1}=0.5\right.$, $\left.a_{2}=0.3, a_{3}=0.25, b_{1}=1.5, c_{1}=0.7, c_{2}=0.5, A=0, a=1.2, m=0.01\right\}$. 


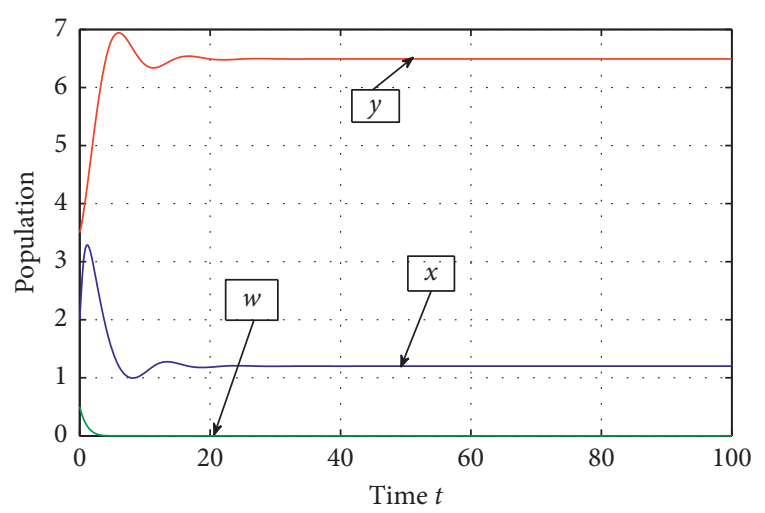

(a)

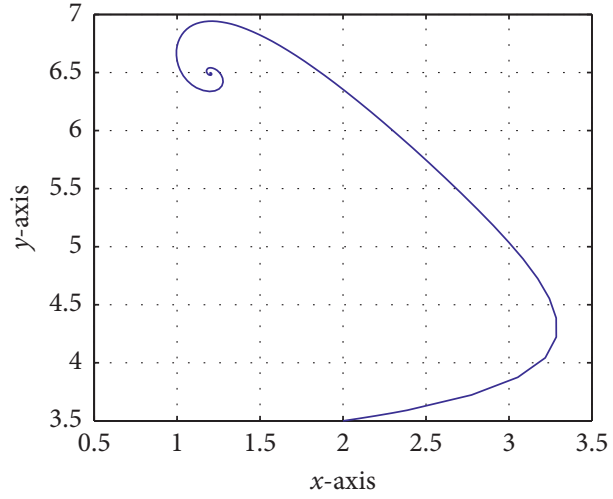

(b)

FIGURE 4: (a) Stable nature of $E_{3}(1.20053,0,6.49267)$ with $t$ and (b) stable phase portrait of $E_{3}(1.20053,0,6.49267)$ when $k=0.2>k^{*}$ (threshold value) $=0.019$ and other are taken as $\left\{r=5.5, d_{1}=0.4, d_{2}=0.3, d_{3}=0.2, a_{1}=0.6, a_{2}=0.98, a_{3}=0.8, b_{1}=2.5, c_{1}=0.85\right.$, $\left.c_{2}=0.7, A=0, a=1.1, m=0.4\right\}$.

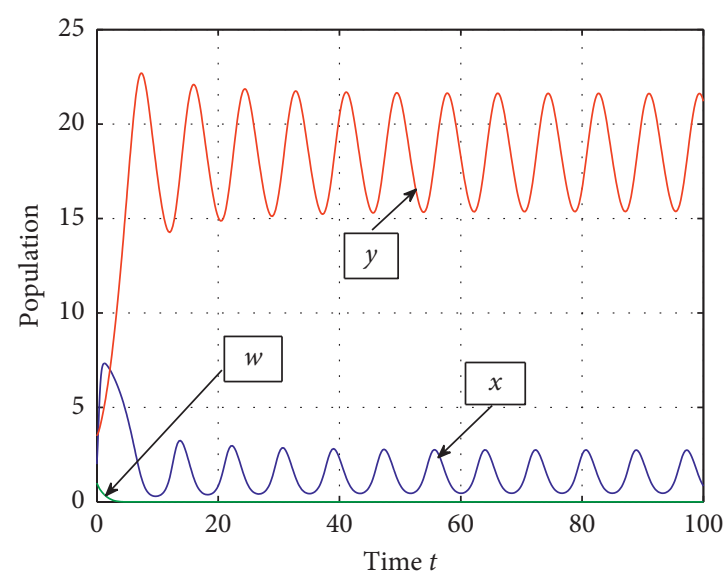

(a)

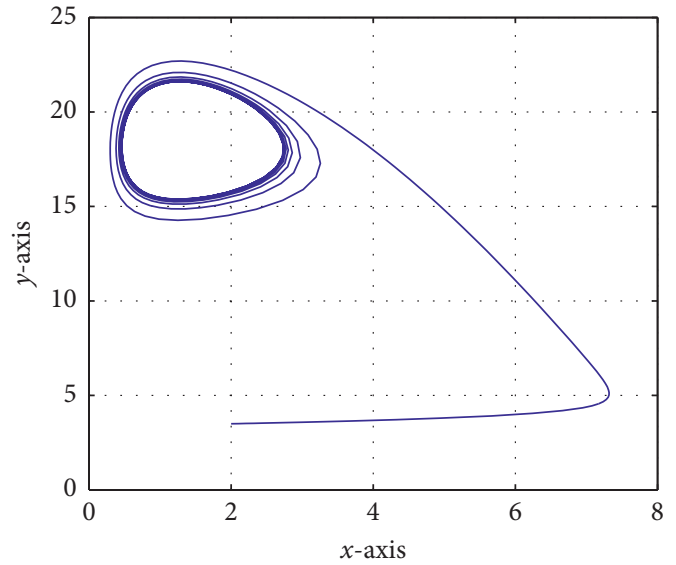

(b)

Figure 5: (a) Oscillatory behaviour of $E_{3}$ with time $t$ and (b) phase diagram (isolated closed orbit) when $k=0.01<k^{*}=0.019$ and all other parameters are fixed as in Figure 4.

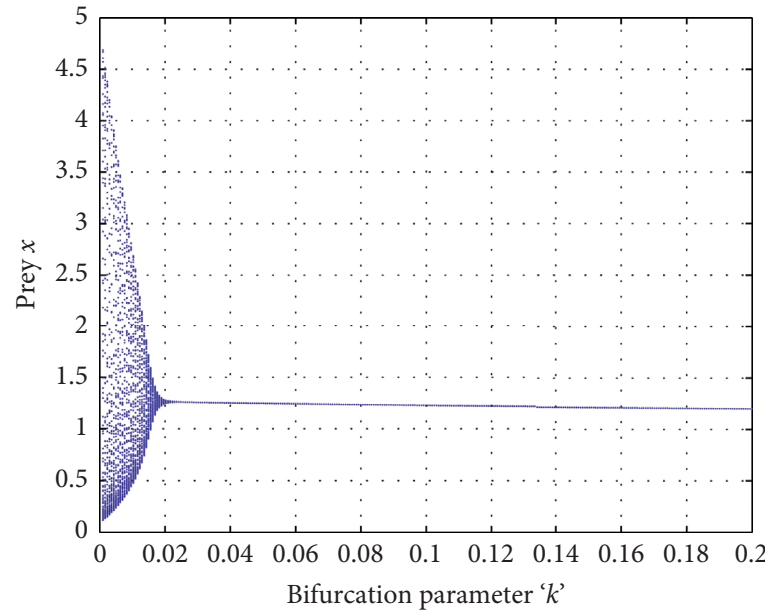

(a)

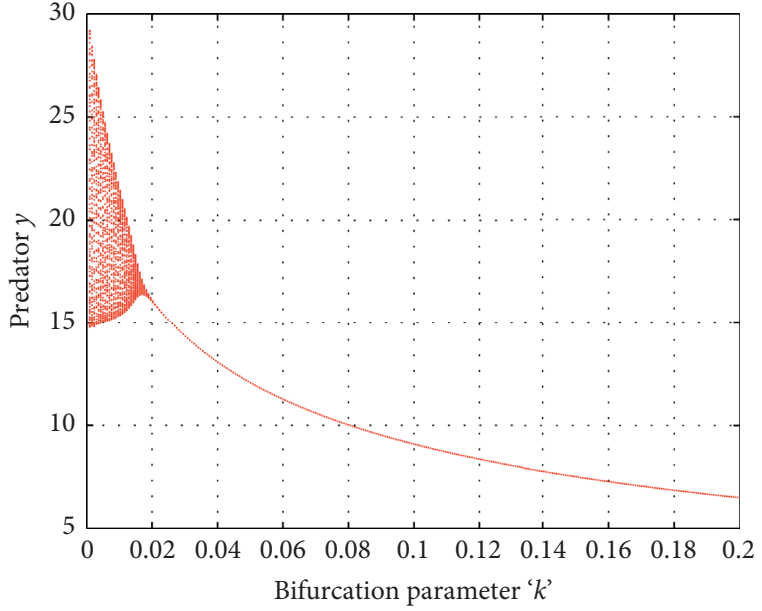

(b)

Figure 6: Bifurcation diagrams for the Hopf bifurcation around $E_{3}(1.20053,0,6.49267)$ regarding $k$ as bifurcation parameter and others are the same as in Figure 4. (a) Bifurcation diagram of $x$. (b) Bifurcation diagram of $y$. 


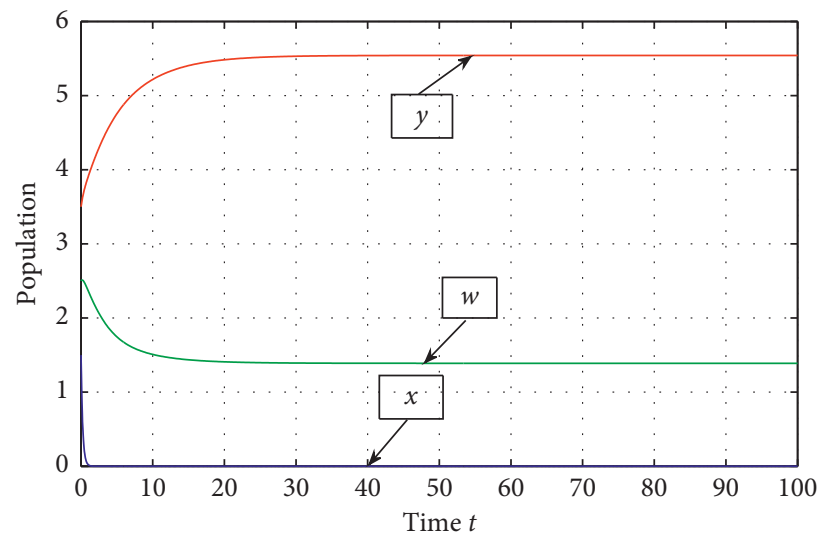

FIGURE 7: Stability nature of $E_{4}(0,1.3889,5.5417)$ with time $t$ regarding the parameters as $\left\{r=5.5, d_{1}=6.5, d_{2}=0.3, k=0.2, d_{3}=0.2\right.$, $\left.a_{1}=0.6, a_{2}=0.98, a_{3}=0.8, b_{1}=2.5, c_{1}=0.85, c_{2}=0.7, A=2, a=1.1, m=0.01\right\}$.

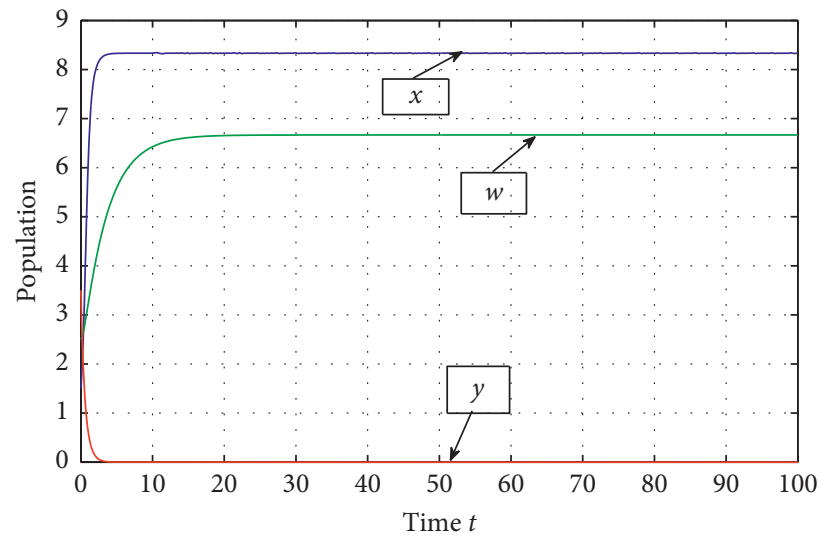

FIGURE 8: Stability nature of $E_{5}(8.3333,6.6667,0)$ with time $t$ regarding the parameters as $\left\{r=5.5, d_{1}=0.5, d_{2}=0.3, k=0.2, d_{3}=2.2\right.$, $\left.a_{1}=0.6, a_{2}=0.98, a_{3}=0.8, b_{1}=2.5, c_{1}=0.85, c_{2}=0.7, A=2, a=1.1, m=0.01\right\}$.

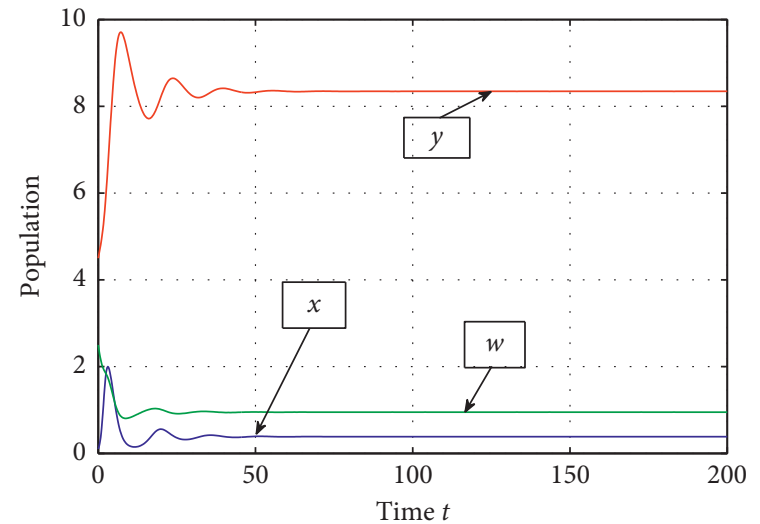

(a)

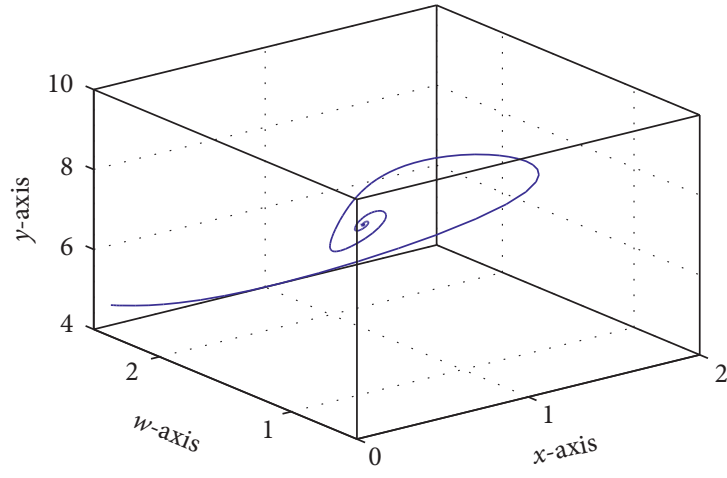

(b)

FiguRE 9: (a) Stable nature of $E^{*}(0.385717,0.950363,8.34509)$ with time $t$ and (b) stable phase diagram of $E^{*}(0.385717,0.950363,8.34509)$ when $k=0.2>k^{[H]}=0.025$ and others are chosen as $\left\{r=5.5, d_{1}=0.4, d_{2}=0.3, d_{3}=0.2, a_{1}=0.6, a_{2}=0.98, a_{3}=0.8, b_{1}=2.5, c_{1}=0.85\right.$, $\left.c_{2}=0.7, A=2, a=1.1, m=0.4\right\}$. 


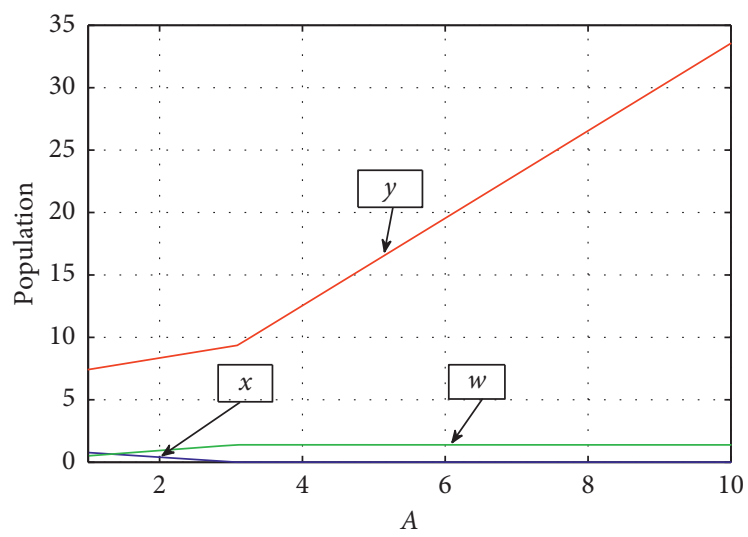

FIgURE 10: Nature of steady state $E^{*}$ when subsidy input rate $A$ varies from 1 to 10 and other parameters are fixed as in Figure 9.

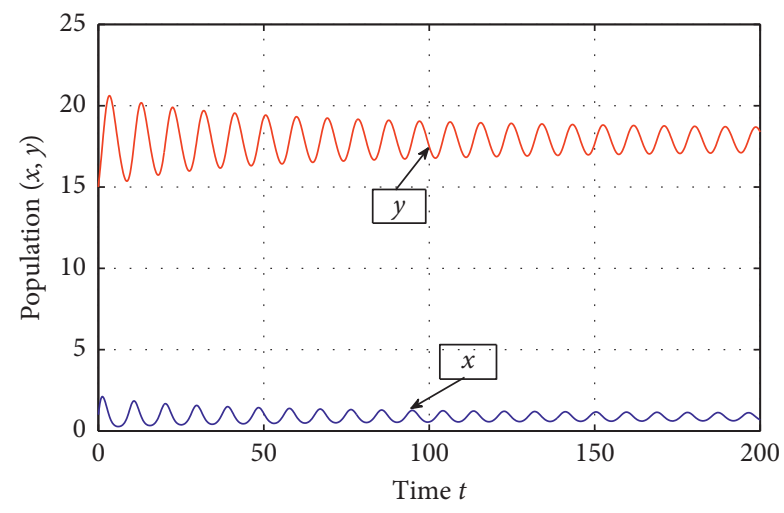

(a)

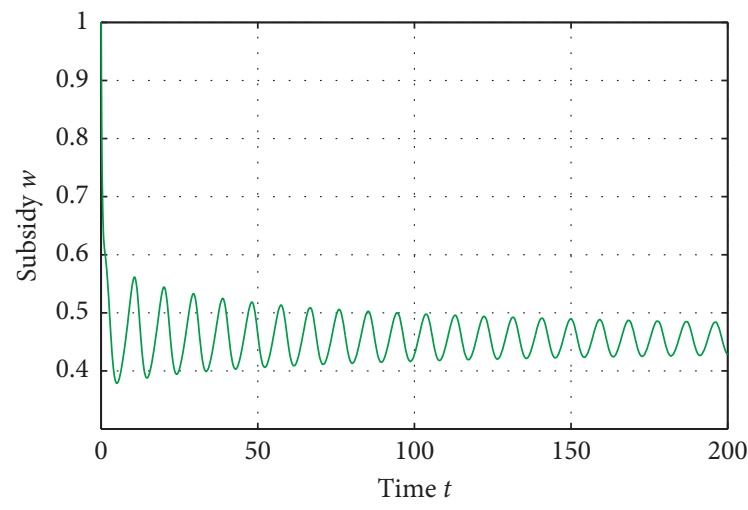

(b)

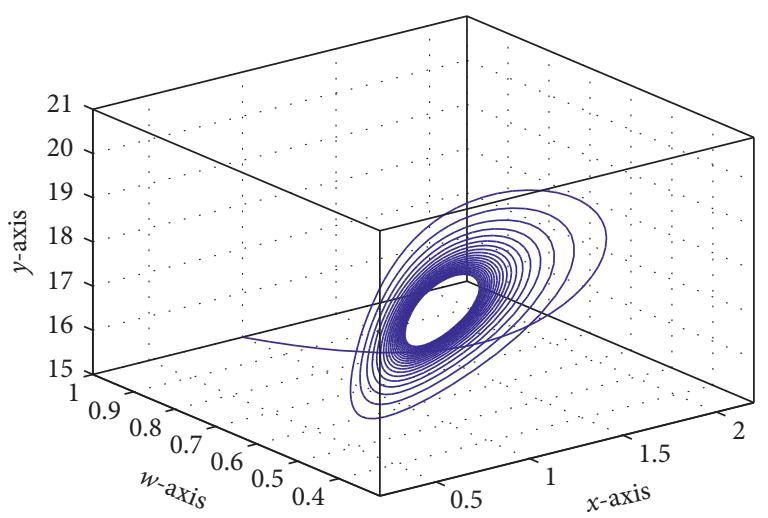

(c)

FIgURE 11: (a), (b) Oscillatory behaviour of $E^{*}$ with time and (c) isolated closed orbit around $E^{*}$ when $k=0.02<k^{[H]}=0.025$ and all others are fixed as in Figure 9.

$$
\begin{aligned}
K^{\prime \prime} & =\left\{-3 \sigma_{+}+R_{2}+S_{1} \cos \left(\sqrt{\sigma_{+}} \tau^{*}\right)-S_{2} \tau^{*} \cos \left(\sqrt{\sigma_{+}} \tau^{*}\right)-S_{1} \sqrt{\sigma_{+}} \tau^{*} \sin \left(\sqrt{\sigma_{+}} \tau^{*}\right)\right\}, \\
L^{\prime \prime} & =\left\{-2 R_{1} \sqrt{\sigma_{+}}+S_{1} \sin \left(\sqrt{\sigma_{+}} \tau^{*}\right)-S_{2} \tau^{*} \sin \left(\sqrt{\sigma_{+}} \tau^{*}\right)+S_{1} \sqrt{\sigma_{+}} \tau^{*} \cos \left(\sqrt{\sigma_{+}} \tau^{*}\right)\right\}, \\
M^{\prime \prime} & =\left\{S_{2} \sqrt{\sigma_{+}} \sin \left(\sqrt{\sigma_{+}} \tau^{*}\right)-S_{1} \sigma_{+} \cos \left(\sqrt{\sigma_{+}} \tau^{*}\right)\right\}, \\
N^{\prime \prime} & =\left\{S_{2} \sqrt{\sigma_{+}} \cos \left(\sqrt{\sigma_{+}} \tau^{*}\right)+S_{1} \sigma_{+} \sin \left(\sqrt{\sigma_{+}} \tau^{*}\right)\right\} .
\end{aligned}
$$

Proof. Proof is similar to that in Theorem 18. 


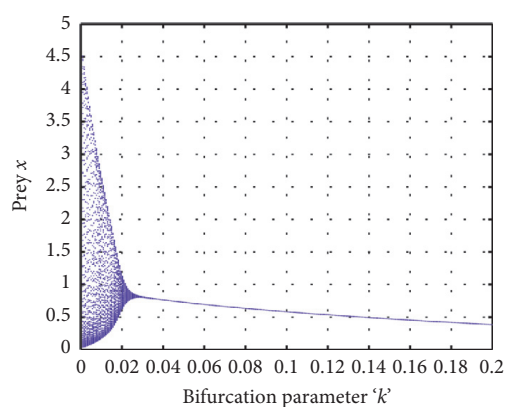

(a)

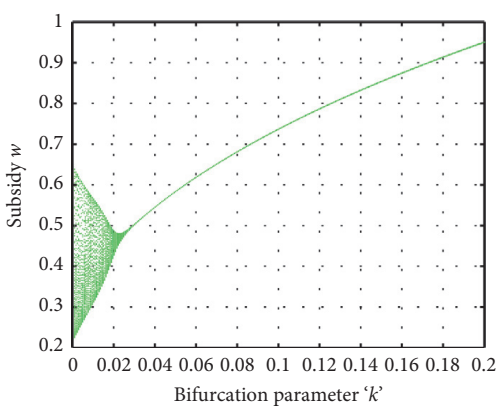

(b)

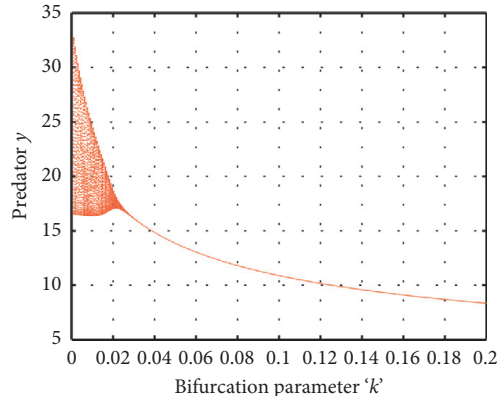

(c)

FIgURE 12: Bifurcation diagrams for the Hopf bifurcation around $E^{*}(0.385717,0.950363,8.34509)$ regarding $k$ as bifurcation parameter and others are the same as in Figure 9. (a) Bifurcation diagram of $x$. (b) Bifurcation diagram of $w$. (c) Bifurcation diagram of $y$.

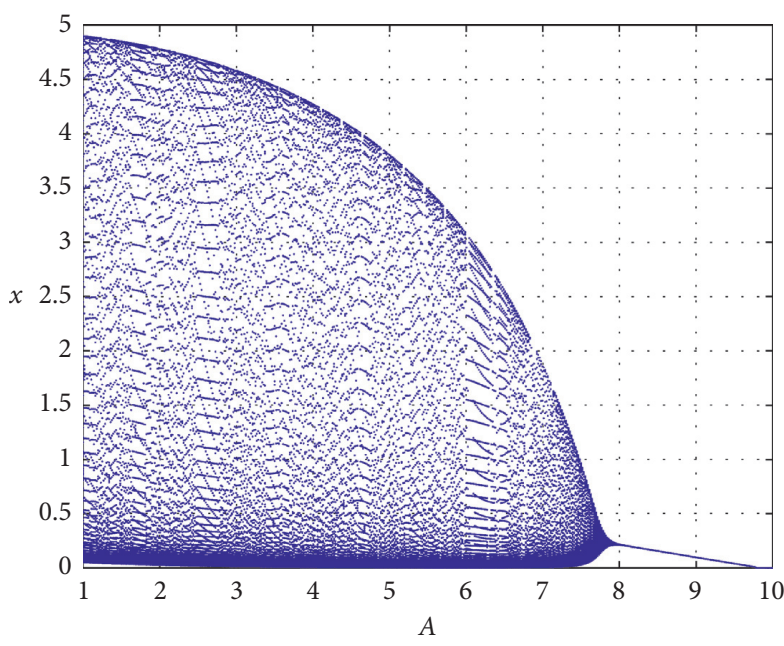

(a)

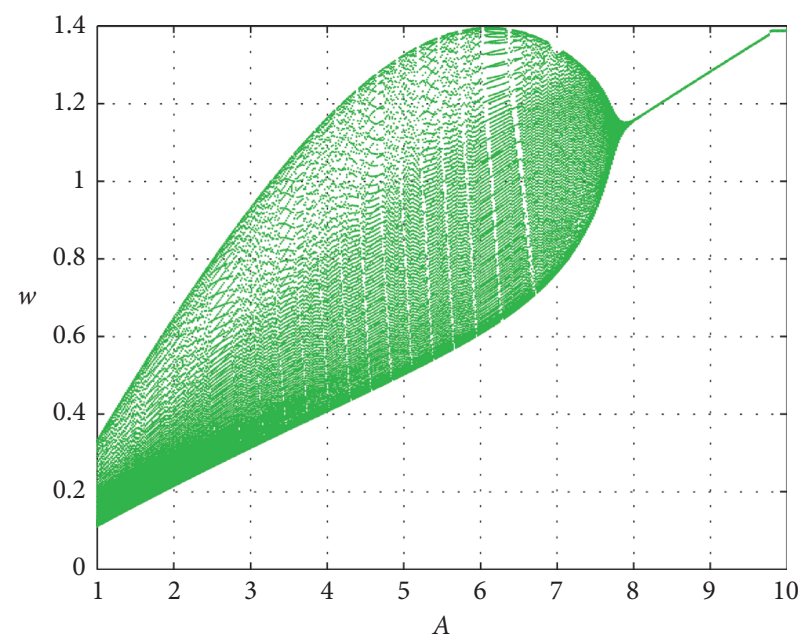

(b)

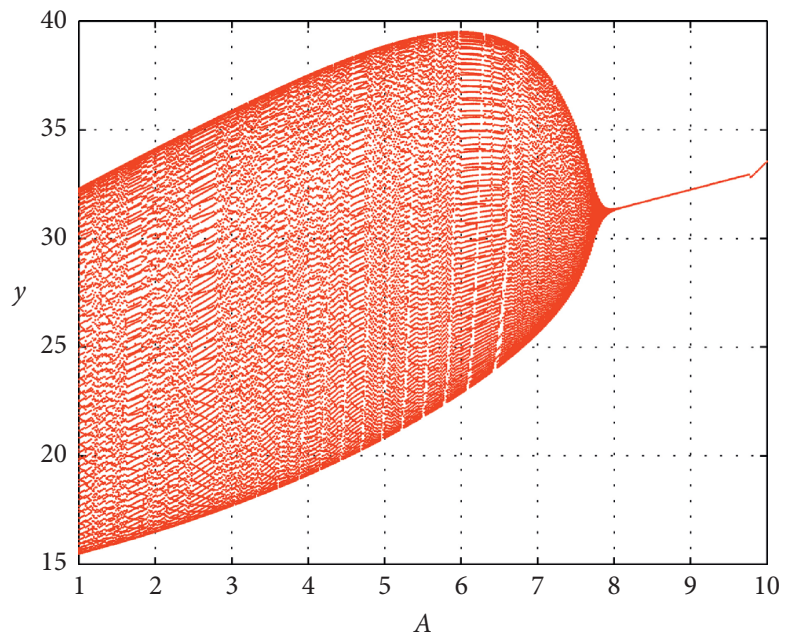

(c)

Figure 13: Bifurcation diagram with respect to subsidy input rate $A$ when $k=0$ and the remaining parameters are fixed as in Figure 9. (a) Bifurcation diagram of $x$. (b) Bifurcation diagram of $w$. (c) Bifurcation diagram of $y$. 


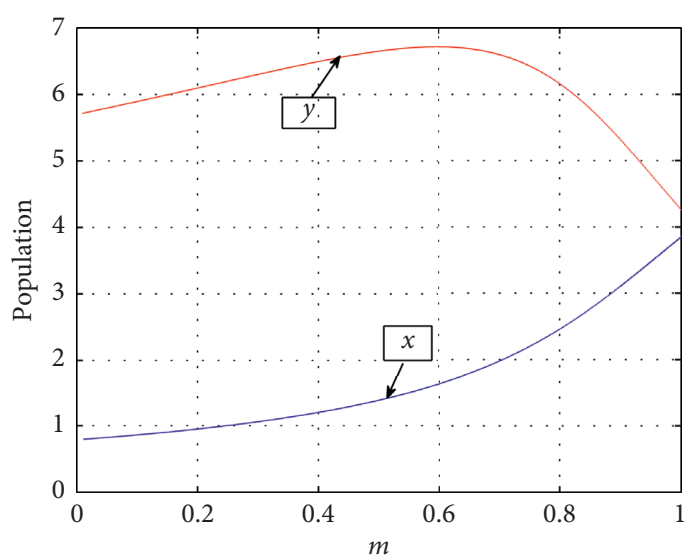

(a)

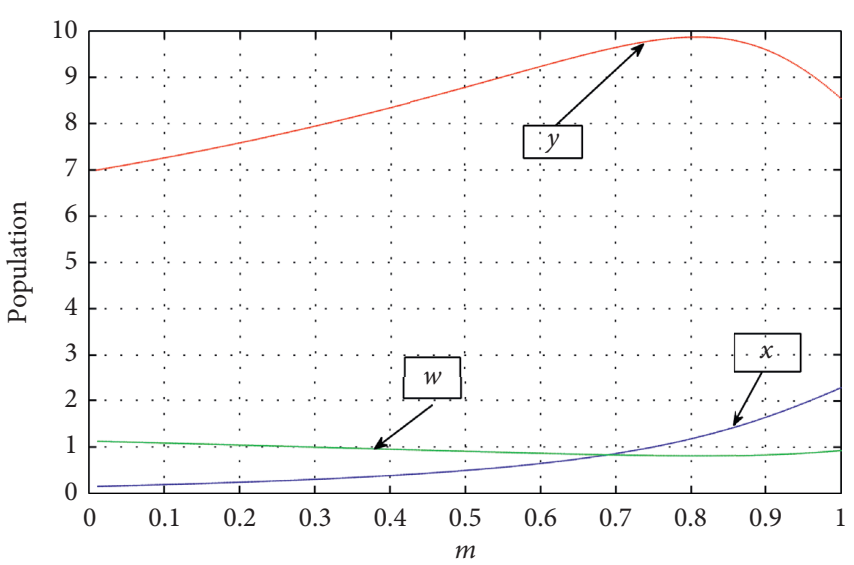

(b)

Figure 14: Impact of $m$ on the nature of steady states $E_{3}$ and $E^{*}$ when other parameters are fixed as in Figure 9. (a) Nature of steady state $E_{3}$ $(A=0)$. (b) Nature of steady state $E^{*}(A \neq 0)$.

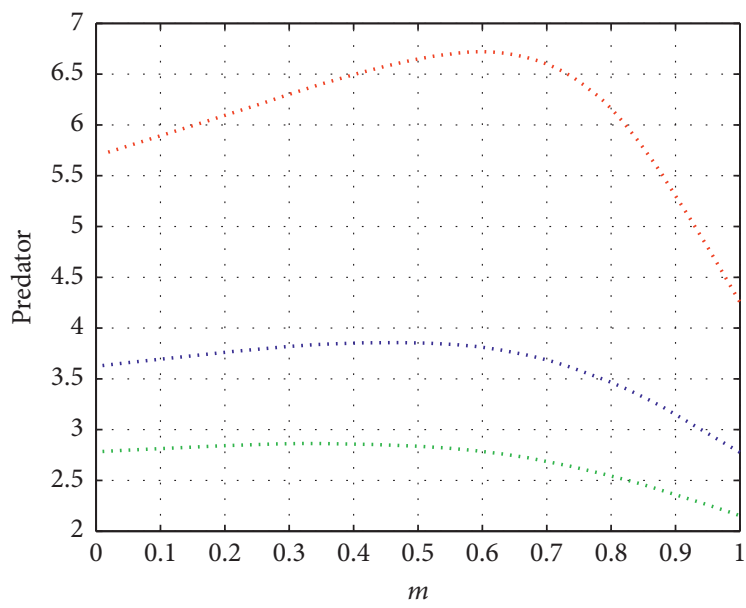

(a)

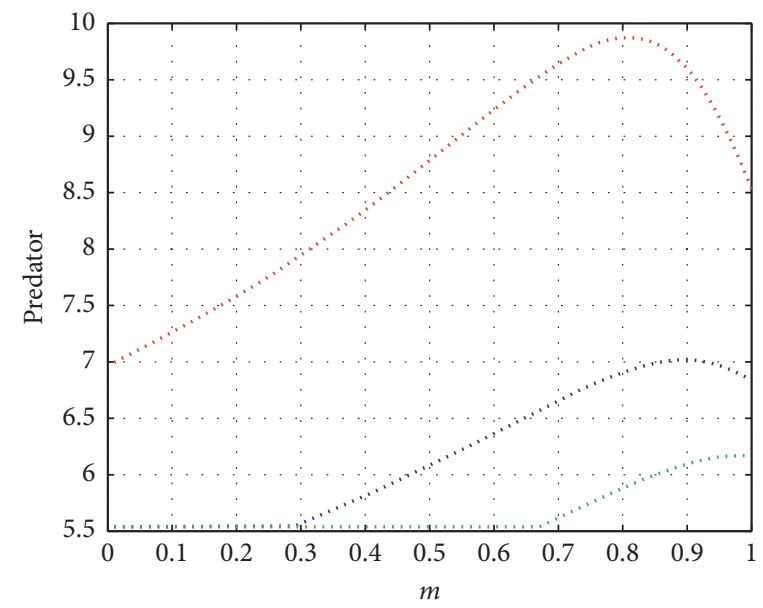

(b)

FIGURE 15: Impact of $m$ on the predator's growth for different values of $k$ when others are fixed as in Figure 9. ((i) red color: $k=0.2$, (ii) blue color: $k=0.5$, and (iii) green color: $k=0.8$ ). (a) Nature at $E_{3}(A=0)$. (b) Nature at $E^{*}(A \neq 0)$.

Theorem 20. Suppose interior (coexistence) equilibrium point $E^{*}$ exists and is locally asymptotically stable for system (2) when $\tau=0$. Let equation (91) have exactly two positive roots $\sigma_{i}, i=1,2\left(\sigma_{1}>\sigma_{2}\right) \quad$ when $\quad R_{3}^{2}>S_{2}^{2}$ and $R_{2}^{2}-2 R_{1} R_{3}-S_{1}^{2}<0$ irrespective of sign of $R_{1}^{2}-2 R_{2}$. Moreover, let

$$
\begin{gathered}
\tau_{j}^{i}=\frac{\cos ^{-1}\left(\left(S_{2}\left(R_{1} \sigma_{i}-R_{3}\right)+S_{1}\left(\sigma_{i}^{2}-R_{2} \sigma_{i}\right)\right) /\left(S_{2}^{2}+S_{1}^{2} \sigma_{i}\right)\right)}{\sqrt{\sigma_{i}}}+\frac{2 \pi j}{\sqrt{\sigma_{i}}}, \quad i=1,2 ; j=0,1,2,3 \ldots, \\
\tau_{k}^{+}=\min \left\{\tau_{k}^{i}: i=1,2\right\}, \tau_{k}^{-}=\max \left\{\tau_{k}^{i}: i=1,2\right\}, \quad k=0,1,2,3 \ldots, \\
\tau \in\left[0, \tau_{0}^{+}\right),\left(\tau_{0}^{-}, \tau_{1}^{+}\right),\left(\tau_{1}^{-}, \tau_{2}^{+}\right), \ldots,\left(\tau_{k-1}^{-}, \tau_{k}^{+}\right),
\end{gathered}
$$

then there is a positive integer $k$ such that

$$
0<\tau_{0}^{+}<\tau_{0}^{-}<\tau_{1}^{+}<\tau_{1}^{-}<\tau_{2}^{+}<\cdots<\tau_{k-1}^{-}<\tau_{k}^{+},
$$

and there are $k$ switches from stability to instability to stability; that is, when then $E^{*}$ is locally asymptotically stable and when

$$
\tau \in\left(\tau_{0}^{+}, \tau_{0}^{-}\right),\left(\tau_{1}^{+}, \tau_{1}^{-}\right),\left(\tau_{2}^{+}, \tau_{2}^{-}\right), \ldots,\left(\tau_{k-1}^{+}, \tau_{k-1}^{-}\right), \tau>\tau_{k}^{+},
$$




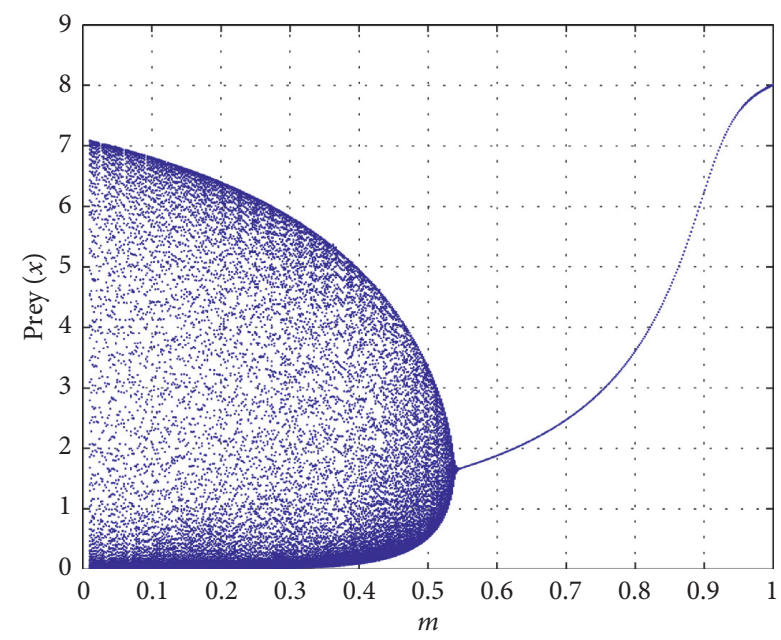

(a)

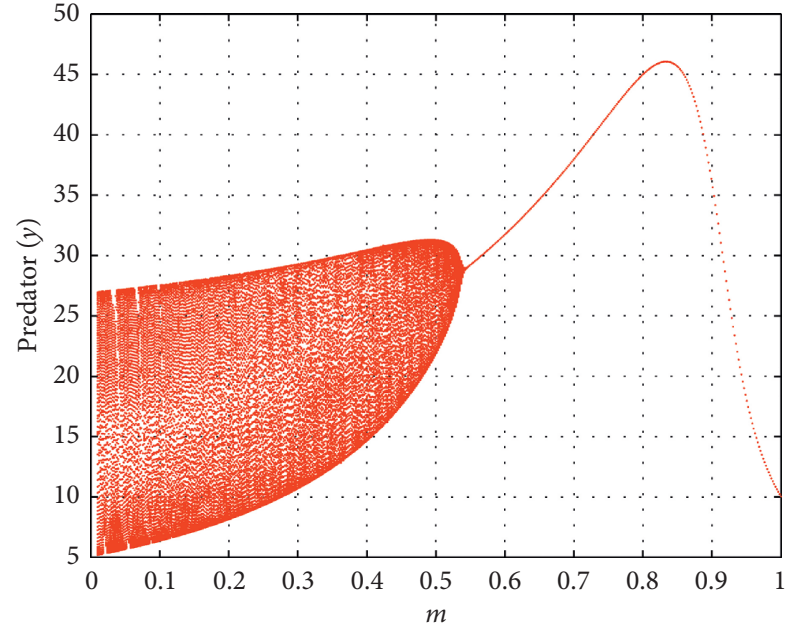

(b)

FIGURE 16: Bifurcation diagram around $E_{3}$ with respect to coefficient of refuge parameter $m$ when $k=0$ and the remaining parameters are fixed as in Figure 4. (a) Bifurcation diagram of $x$. (b) Bifurcation diagram of $y$.

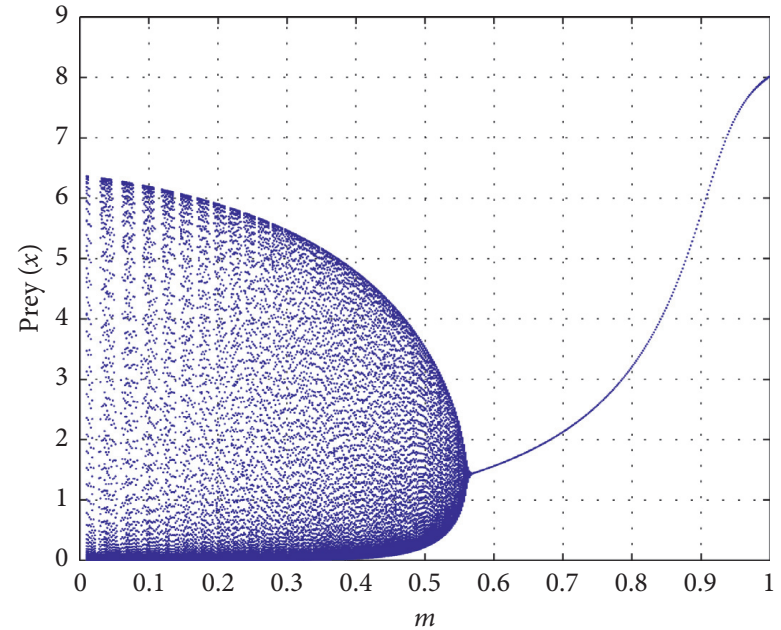

(a)

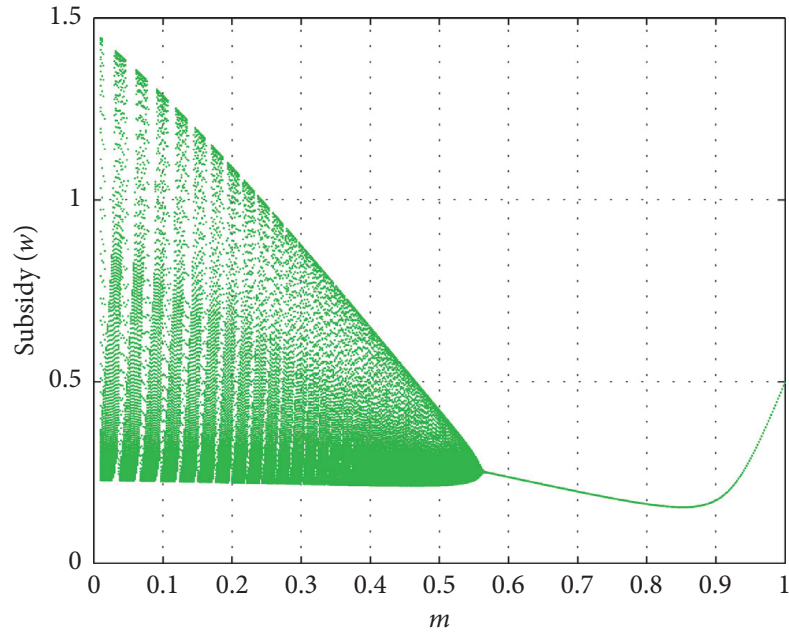

(b)

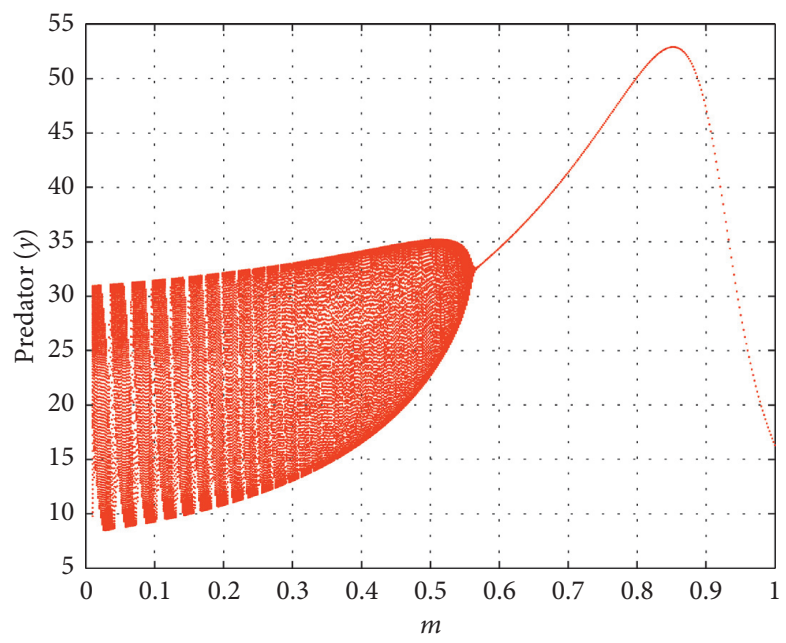

(c)

FIgURE 17: Bifurcation diagram around $E^{*}$ when $k=0$ and the remaining parameters are fixed as in Figure 9. (a) Bifurcation diagram of $x$. (b) Bifurcation diagram of $w$. (c) Bifurcation diagram of $y$. 


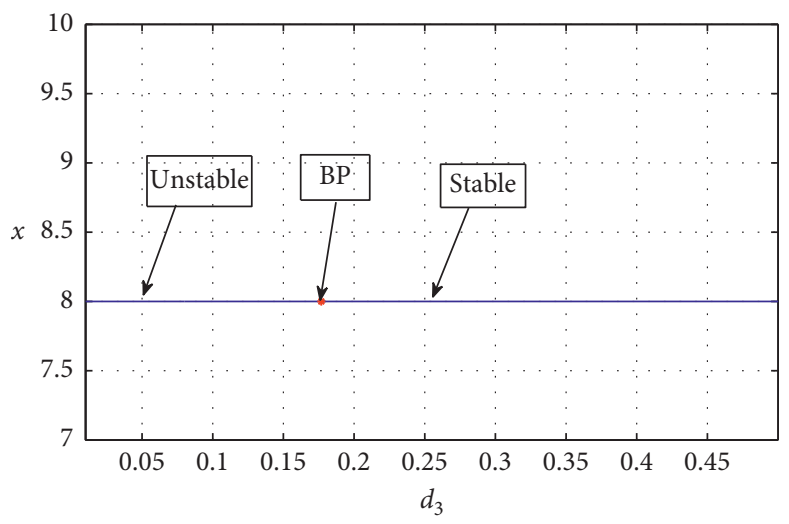

Figure 18: Transcritical bifurcation diagram around $E_{2}(8.0,0,0)$ considering $d_{3}$ as bifurcation parameter and others parameters are the same as in Figure 3. Here $d_{3}^{[\mathrm{TC}]}=0.1768$.

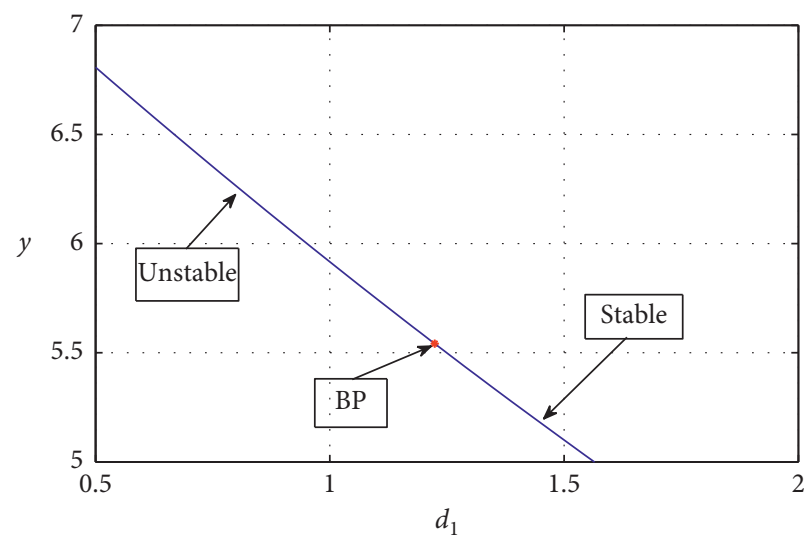

FiguRE 19: Bifurcation diagram for the transcritical bifurcation around $E_{4}(0,1.9889,5.5417)$ considering $d_{1}$ as the independent variable and others parameters are the same as in Figure 7 . Here, $d_{1}^{[\mathrm{TC}]}=1.2238$.

then $E^{*}$ is unstable. Further, at $\tau=\tau_{k}^{ \pm}, k=0,1,2, \ldots$, system where (60) experiences Hopf bifurcation provided

$$
\begin{array}{ll}
P_{k}^{+} R_{k}^{+}-Q_{k}^{+} S_{k}^{+} \neq 0, & \text { for } k=0,1,2, \ldots, \\
P_{k}^{-} R_{k}^{-}-Q_{k}^{-} S_{k}^{-} \neq 0, & \text { for } k=0,1,2, \ldots,
\end{array}
$$

$$
\begin{aligned}
P_{k}^{+} & =\left\{-3 \sigma_{1}+R_{2}+S_{1} \cos \left(\sqrt{\sigma_{1}} \tau_{k}^{+}\right)-S_{2} \tau_{k}^{+} \cos \left(\sqrt{\sigma_{1}} \tau_{k}^{+}\right)-S_{1} \sqrt{\sigma_{1}} \tau_{k}^{+} \sin \left(\sqrt{\sigma_{1}} \tau_{k}^{+}\right)\right\}_{k=0,1,2, \ldots}, \\
P_{k}^{-} & =\left\{-3 \sigma_{2}+R_{2}+S_{1} \cos \left(\sqrt{\sigma_{2}} \tau_{k}^{-}\right)-S_{2} \tau_{k}^{-} \cos \left(\sqrt{\sigma_{2}} \tau_{k}^{-}\right)-S_{1} \sqrt{\sigma_{2}} \tau_{k}^{-} \sin \left(\sqrt{\sigma_{2}} \tau_{k}^{-}\right)\right\}_{k=0,1,2, \ldots}, \\
Q_{k}^{+} & =\left\{-2 R_{1} \sqrt{\sigma_{1}}+S_{1} \sin \left(\sqrt{\sigma_{1}} \tau_{k}^{+}\right)-S_{2} \tau_{k}^{+} \sin \left(\sqrt{\sigma_{1}} \tau_{k}^{+}\right)+S_{1} \sqrt{\sigma_{1}} \tau_{k}^{+} \cos \left(\sqrt{\sigma_{1}} \tau_{k}^{+}\right)\right\}_{k=0,1,2, \ldots}, \\
Q_{k}^{-} & =\left\{-2 R_{1} \sqrt{\sigma_{2}}+S_{1} \sin \left(\sqrt{\sigma_{2}} \tau_{k}^{-}\right)-S_{2} \tau_{k}^{-} \sin \left(\sqrt{\sigma_{2}} \tau_{k}^{-}\right)+S_{1} \sqrt{\sigma_{2}} \tau_{k}^{-} \cos \left(\sqrt{\sigma_{2}} \tau_{k}^{-}\right)\right\}_{k=0,1,2, \ldots}, \\
R_{k}^{+} & =\left\{S_{2} \sqrt{\sigma_{1}} \sin \left(\sqrt{\sigma_{1}} \tau_{k}^{+}\right)-S_{1} \sigma_{1} \cos \left(\sqrt{\sigma_{1}} \tau_{k}^{+}\right)\right\}_{k=0,1,2, \ldots}, \\
R_{k}^{-} & =\left\{S_{2} \sqrt{\sigma_{2}} \sin \left(\sqrt{\sigma_{2}} \tau_{k}^{-}\right)-S_{1} \sigma_{2} \cos \left(\sqrt{\sigma_{2}} \tau_{k}^{-}\right)\right\}_{k=0,1,2, \ldots}, \\
S_{k}^{+} & =\left\{S_{2} \sqrt{\sigma_{1}} \cos \left(\sqrt{\sigma_{1}} \tau_{k}^{+}\right)+S_{1} \sigma_{1} \sin \left(\sqrt{\sigma_{1}} \tau_{k}^{+}\right)\right\}_{k=0,1,2, \ldots}, \\
S_{k}^{-} & =\left\{S_{2} \sqrt{\sigma_{2}} \cos \left(\sqrt{\sigma_{2}} \tau_{k}^{-}\right)+S_{1} \sigma_{2} \sin \left(\sqrt{\sigma_{2}} \tau_{k}^{-}\right)\right\}_{k=0,1,2, \ldots}
\end{aligned}
$$

Proof. Proof is similar to that in Theorem 18. 


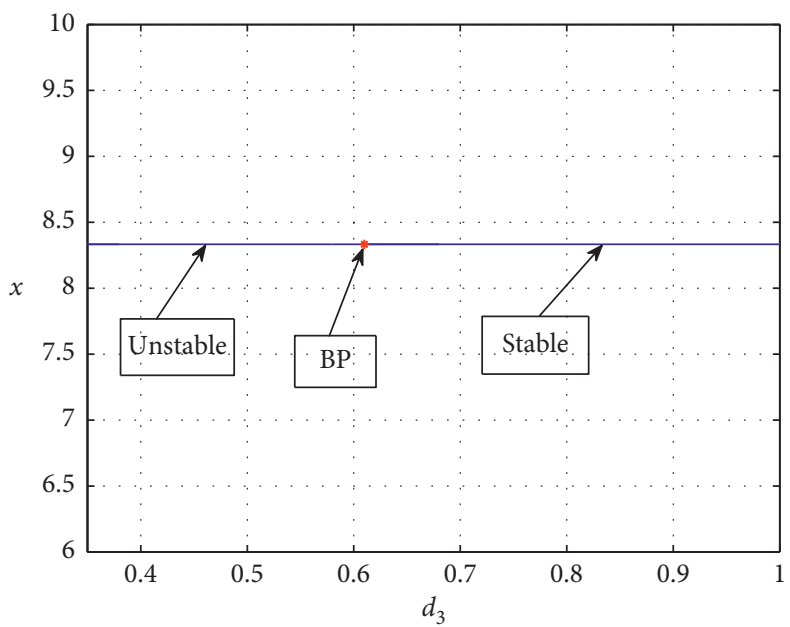

Figure 20: Transcritical bifurcation diagram around $E_{5}(8.3333,6.6667,0)$ considering $d_{3}$ as independent variable and others are the same as in Figure 8. Here, $d_{3}^{[\mathrm{TC}]}=0.61$.

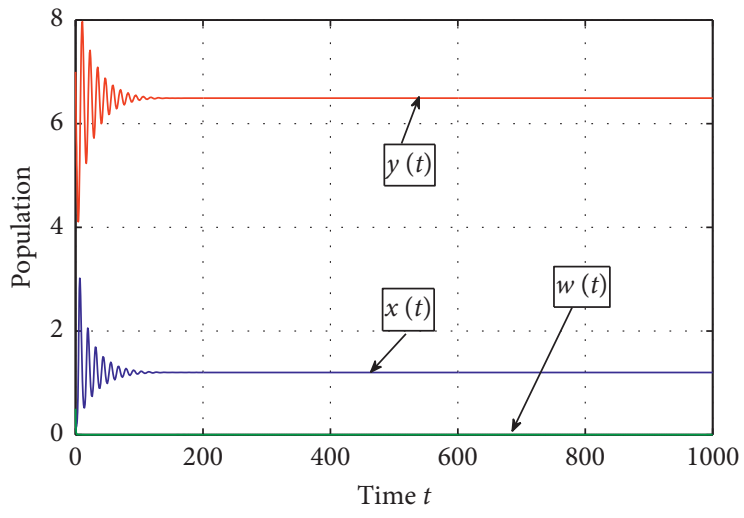

(a)

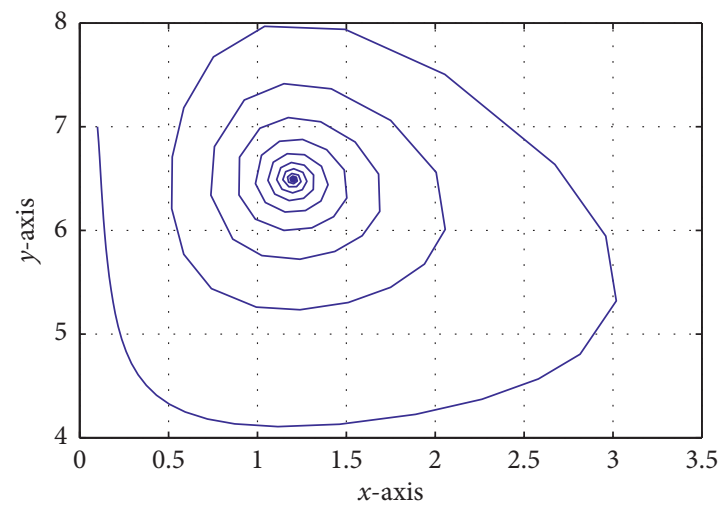

(b)

Figure 21: (a) Stable nature with time and (b) stable spiral of $E_{3}(1.20053,0,6.49267)$ when $\tau=2<\tau^{\prime *}=2.9272$ corresponding to the data set of Figure 4.

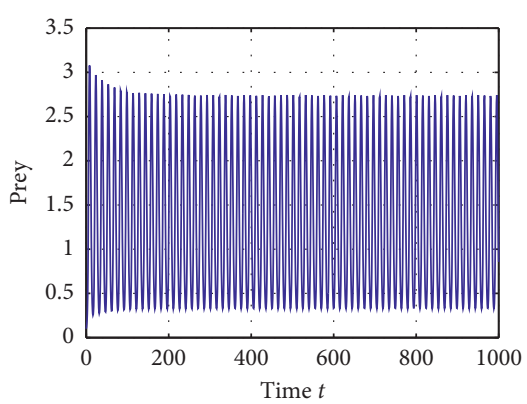

(a)

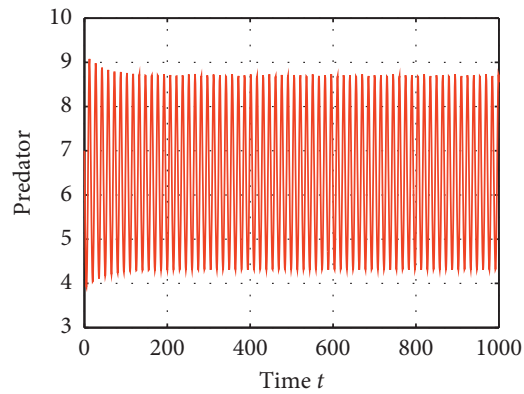

(b)

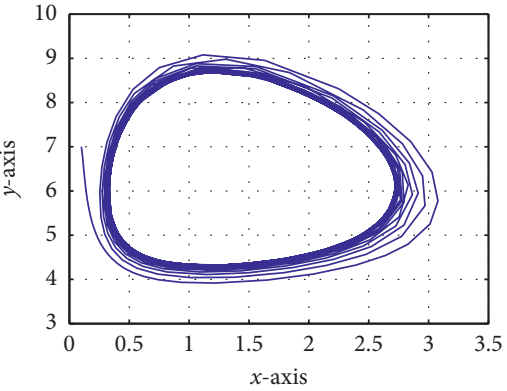

(c)

Figure 22: (a, b) Oscillatory nature of $x, y$, respectively, and (c) stable limit cycle around $E_{3}(1.20053,0,6.49267)$ when $\tau=3.5>\tau^{\prime *}=$ 2.9272 corresponding to the data set of Figure 4. (a) Time series of prey. (b) Time series of predator. (c) Phase diagram. 


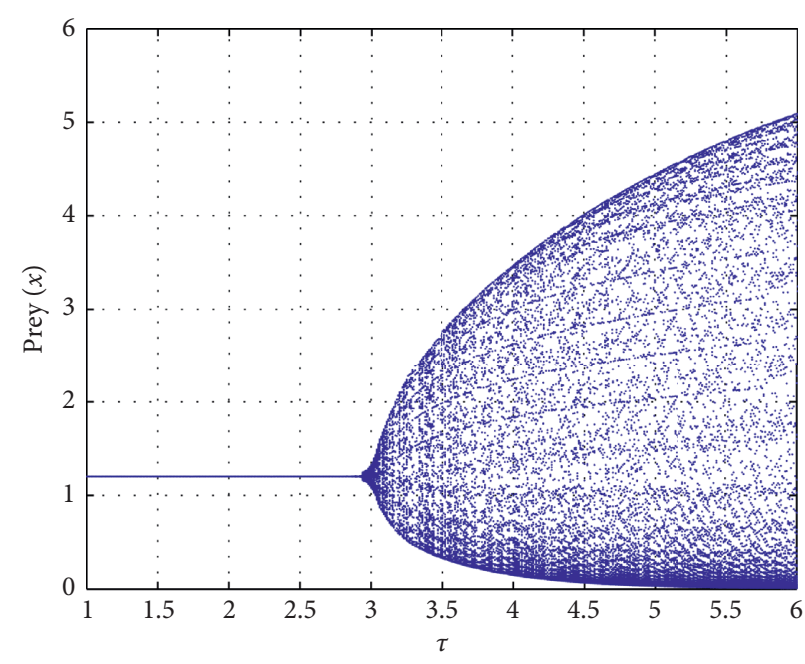

(a)

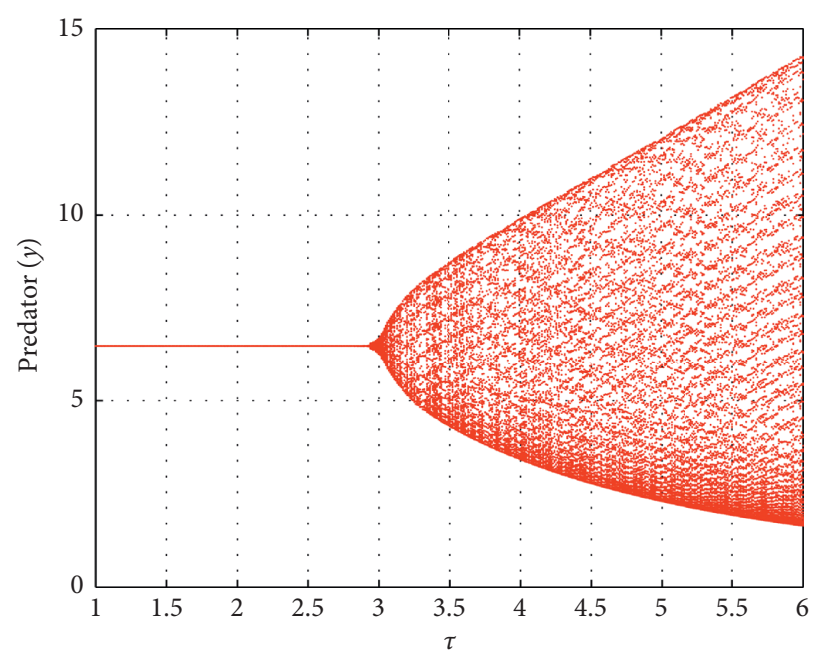

(b)

FIgURE 23: Supercritical Hopf bifurcation diagram around $E_{3}(1.20053,0,6.49267)$ considering $\tau$ as bifurcation parameter and other parameters are chosen from the data set of Figure 4. (a) Bifurcation diagram of prey. (b) Bifurcation diagram of predator.

\section{Numerical Computations}

Here, we have illustrated numerical simulations to verify the analytical findings of the proposed system (2). We select a parameter set: $\left\{r=5.5, d_{1}=6.5, d_{2}=4, k=0.2, d_{3}=5\right.$, $a_{1}=2, a_{2}=0.3, a_{3}=0.25, b_{1}=1.5, c_{1}=0.7, c_{2}=0.5, A=0$, $a=1.2, m=0.01\}$. Under this set of parametric values, the stable nature of $E_{0}(0,0,0)$ is shown in Figure 1 . If we take subsidy input rate $A=2$ and other parametric values are chosen from the data set of Figure 1, then the subsidy only equilibrium $E_{1}\left(0,\left(A / d_{2}\right), 0\right) \equiv E_{1}(0,0.5,0)$ exists and stable nature of $E_{1}(0,0.5,0)$ with time $t$ is depicted in Figure 2. Now, we choose another parameter set: $\left\{r=5.5, d_{1}=1.5\right.$, $d_{2}=0.52, k=1.9, d_{3}=2, a_{1}=0.5, a_{2}=0.3, a_{3}=0.25$, $\left.b_{1}=1.5, c_{1}=0.7, c_{2}=0.5, A=0, a=1.2, m=0.01\right\}$. Under this set of parametric values, the prey only equilibrium $E_{2}\left(\left(r-d_{1}\right) / a_{1}, 0,0\right) \equiv E_{2}(8,0,0)$ exists and stable behaviour of $E_{2}(8,0,0)$ is presented in Figure 3. Let us choose the parameters as follows:

$$
\left\{r=5.5, d_{1}=0.4, d_{2}=0.3, k=0.2, d_{3}=0.2, a_{1}=0.6, a_{2}=0.98, a_{3}=0.8, b_{1}=2.5, c_{1}=0.85, c_{2}=0.7, a=1.1, m=0.4\right\} .
$$

If we take subsidy input rate $A=0$ and other parameters are taken from set (100), then subsidy-free equilibrium point $E_{3}(\bar{x}, 0, \bar{y}) \equiv E_{3}(1.20053,0,6.49267)$ exists and is locally asymptotically stable. Stable time series and stable phase diagram are represented in Figure 4. In the same manner, if we change the value of the parameter $k(=0.01)$ and others are the same as in the data set of Figure 4, then it is observed that $E_{3}(1.20053,0,6.49267)$ is unstable accompanied with a limit cycle (see Figure 5). From Figures 4 and 5, it can be easily noted that there must exist a threshold value of $k$, say $k^{*}=0.019$ for which unstable behaviour of $E_{3}$ changes to stable spiral. Since the vector fields for $k<k^{*}$ and $k>k^{*}$ are qualitatively different, a Hopf bifurcation is created around $E_{3}$ taking $k$ as bifurcation parameter (see Figure 6). For the set of parameter values $\left\{r=5.5, d_{1}=6.5, d_{2}=0.3, k=0.2\right.$, $d_{3}=0.2, a_{1}=0.6, a_{2}=0.98, a_{3}=0.8, b_{1}=2.5, c_{1}=0.85$, $\left.c_{2}=0.7, A=2, a=1.1, m=0.01\right\}$, prey free equilibrium point $E_{4}(0, \widehat{w}, \widehat{y}) \equiv E_{4}(0,1.3889,5.5417)$ exists and is stable (see Figure 7). Next, let us take a different set of parameters of system (2): $\left\{r=5.5, d_{1}=0.5, d_{2}=0.3, k=0.2, d_{3}=2.2\right.$, $a_{1}=0.6, a_{2}=0.98, a_{3}=0.8, b_{1}=2.5, c_{1}=0.85, c_{2}=0.7$, $A=2, a=1.1, m=0.01\}$. Then, predator free equilibrium point $E_{5}\left(\left(r-d_{1}\right) / a_{1},\left(A / d_{2}\right), 0\right) \equiv E_{5}(8.3333,6.6667,0)$ is locally asymptotically stable. The stable behaviour with time $t$ is shown in Figure 8.

If we take subsidy input rate $A=2$ and others are fixed as in the data set of Figure 4, then $E^{*}\left(x^{*}, w^{*}, y^{*}\right) \equiv E^{*}(0.385717,0.950363,8.34509) \quad$ exists and is locally asymptotically stable. Figure 9 depicts the stable behaviour of $E^{*}$. Comparing Figures 4 and 9 , it is observed that subsidy input rate $A$ enhances the value of $y$ component of $E^{*}$ and decreases the value of $x$ component of $E^{*}$. Also, from Figure 10, it is noticed that the prey population is leading towards extinction and the predator population has enormous growth (due to huge supply of food source) at high subsidy input rate (when $A \in(2,10])$ in the presence of fear felt by prey population. So, it can be concluded that it is not possible to control prey population from extinction in presence of nonlinear prey refuge because they cannot get enough time to protect themselves from 


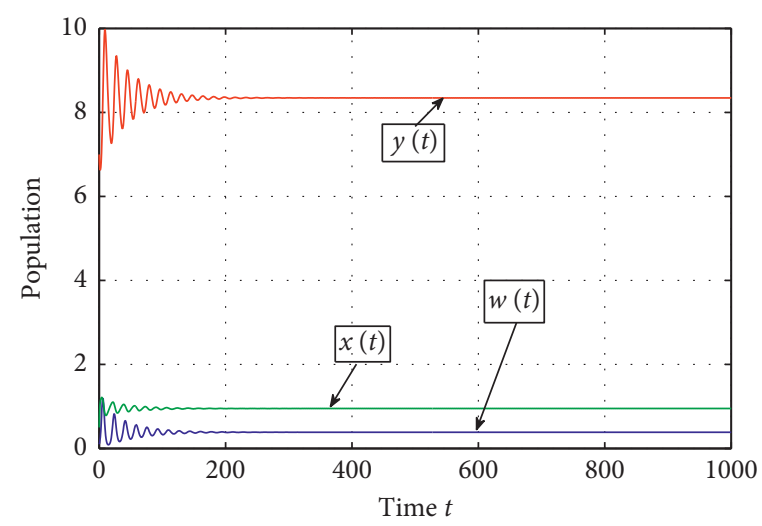

(a)

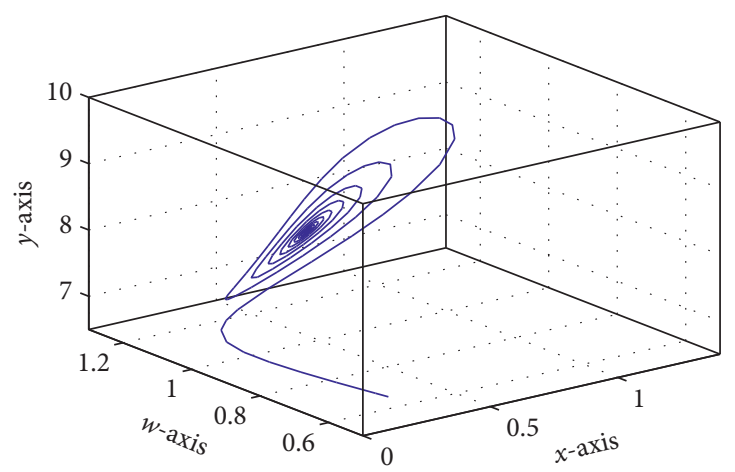

(b)

Figure 24: (a) Stable behaviour with time and (b) stable trajectory of $E^{*}(0.385717,0.950363,8.34509)$ when $\tau=2.5 \epsilon\left[0, \tau_{0}^{+}=4.0652\right)$ corresponding to the data set of Figure 9.

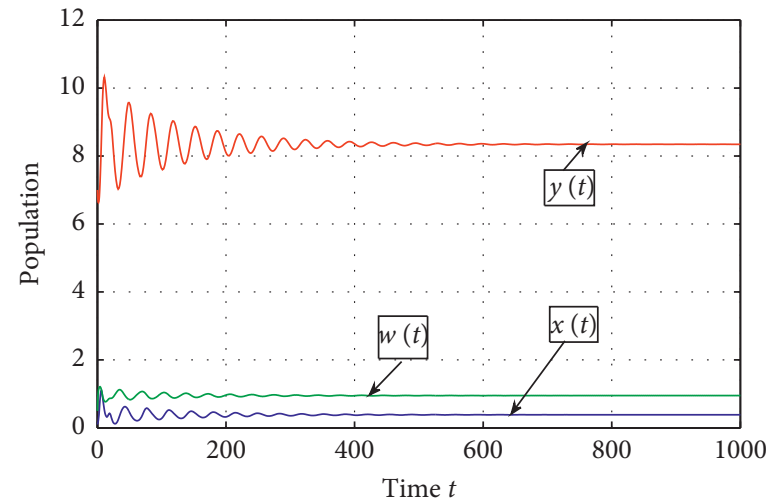

(a)

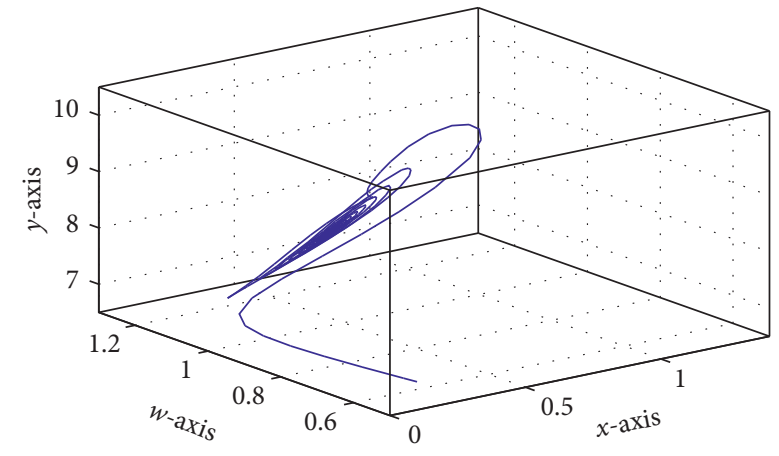

(b)

FIgURE 25: (a) Stable nature with time and (b) stable phase portrait of $E^{*}(0.385717,0.950363,8.34509)$ when $\tau=14.5 \epsilon\left(\tau_{0}^{-}=10.3836, \tau_{1}^{+}=\right.$ 22.7845) corresponding to the data set of Figure 9.

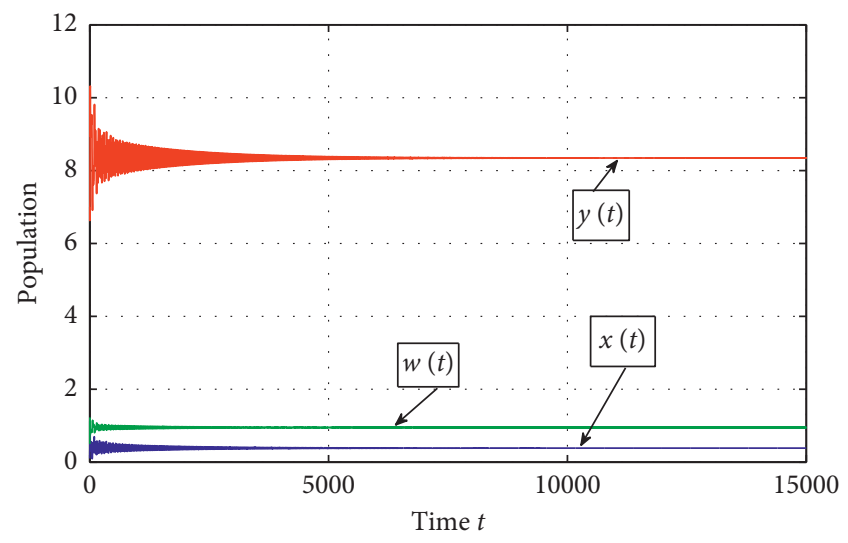

(a)

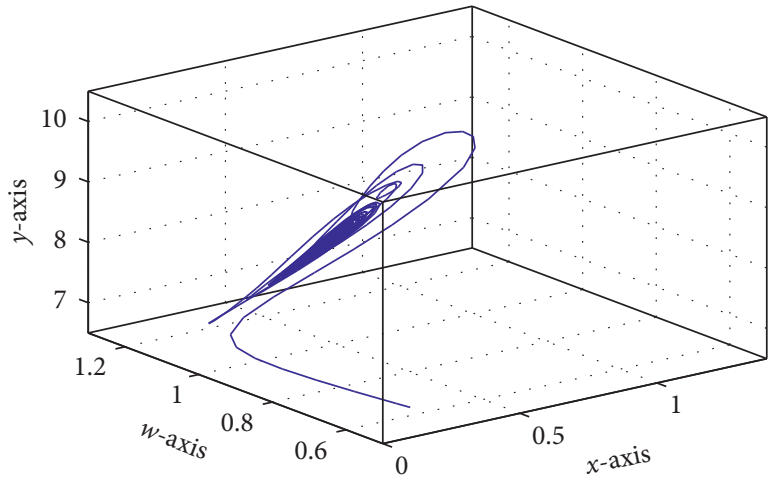

(b)

FiguRE 26: (a) Stable behaviour with time and (b) stable trajectory of $E^{*}(0.385717,0.950363,8.34509)$ when $\tau=39 \in\left(\tau_{1}^{-}=37.7710\right.$, $\tau_{2}^{+}=$ 41.5037) corresponding to the data set of Figure 9. 


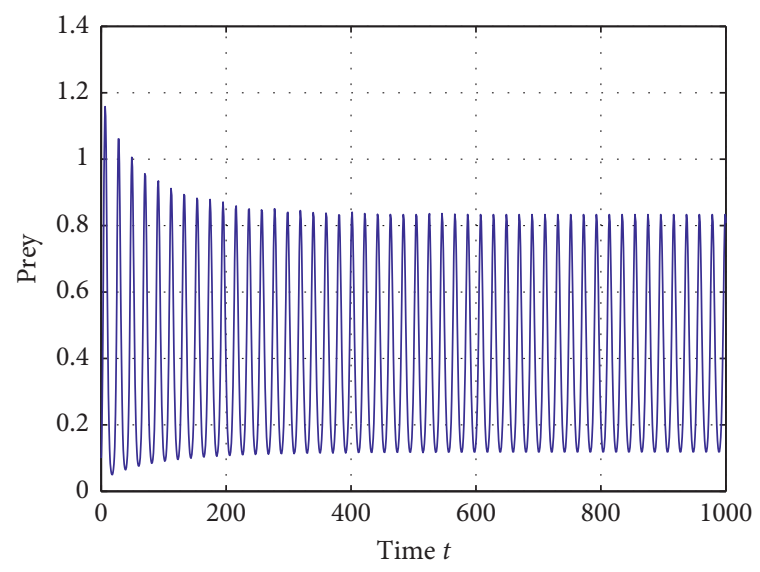

(a)

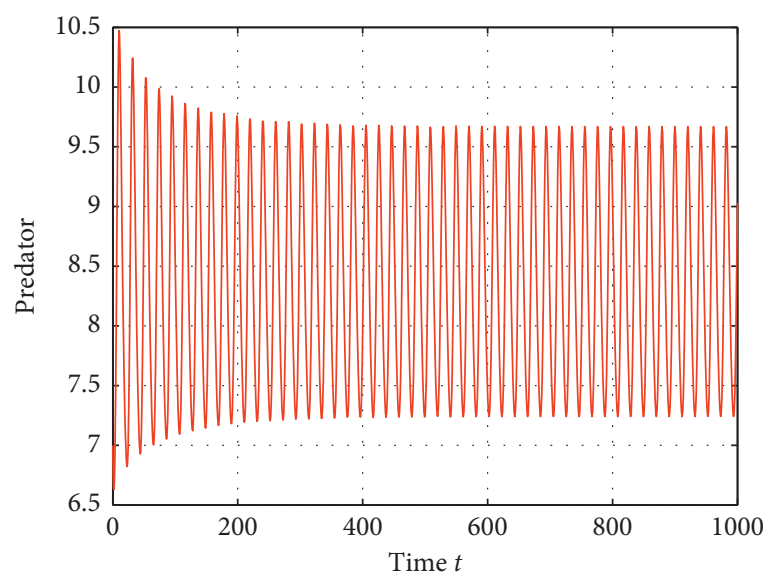

(c)

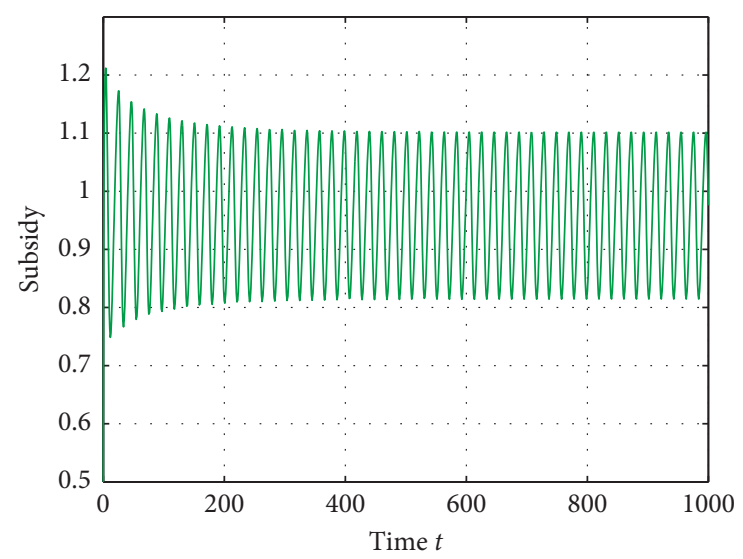

(b)

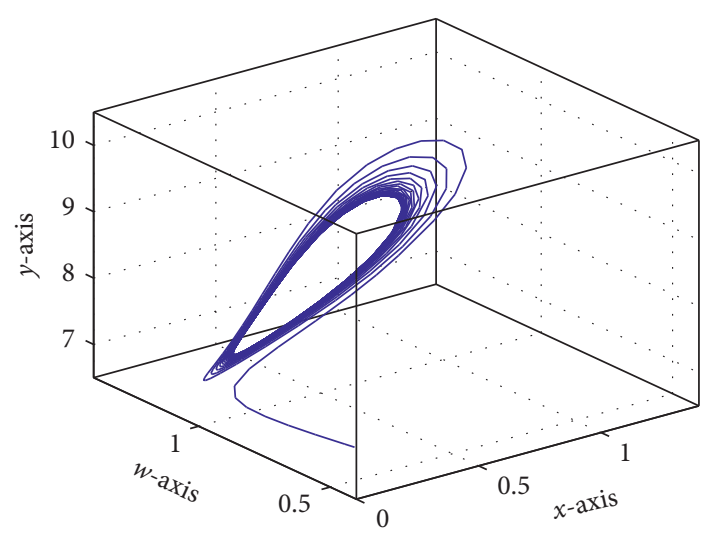

(d)

Figure 27: (a, b, c) Oscillatory nature of prey, subsidy, and predator, respectively, with time and (d) stable limit cycle around $E^{*}(0.385717,0.950363,8.34509)$ when $\tau=5 \epsilon\left(\tau_{0}^{+}=4.0652, \tau_{0}^{-}=10.3836\right)$ corresponding to the data set of Figure 9. (a) Oscillation of prey. (b). Oscillation of subsidy. (c) Oscillation of predator. (d) Phase portrait (closed orbit).

predation risk. After extinction of prey species, predator can easily survive with the help of resource subsidy. Thus, the parameter $A$ has great importance in the proposed population dynamics.

Moreover, Figure 11 represents the unstable nature of $E^{*}$ when $k=0.02$ and other parametric values are the same as in Figure 9. So, the parameter $k$ has an interesting nature because there exists a threshold value $k^{[H]}=0.025$ of $k$ for which unstable nature (limit cycle) of $E^{*}$ switches to stable behaviour (stable spiral) when $k$ passes through its critical value $k^{[H]}$; i.e., the vector fields for $k>k^{[H]}$ and $k<k^{[H]}$ are topologically different. Hence, a Hopf bifurcation occurs around $E^{*}$ and Figure 12 depicts the corresponding bifurcation diagram taking $k$ as bifurcation parameter. Also, it has to be noted from Figure 13 that, in the absence of fear effect, the oscillatory behaviour of $E^{*}$ changes to stable nature when subsidy input rate $A$ crosses its critical value $A^{*}=7.9$ (approximately) and since predator population has huge growth rate at very large value of subsidy input rate $A$, the prey population cannot persist in ecosystem in presence nonlinear prey refuge. This phenomenon is very interesting because the prey refuge cannot control the prey population from extinction due to enormous growth of predator when subsidy input rate is very high. In this manner, system (2) is not persistent.

Further, Figure 14 depicts that the nature of steady states $E_{3}$ and $E^{*}$ when $m \in(0,1)$. Here, the predator population cannot go extinct for large value of coefficient of prey refuge parameter. Also, Figure 15 shows the changes of predator's growth at the steady states $E_{3}$ and $E^{*}$ for three different fear levels $k$ when $m$ varies from 0 to 1 . Here, also the predator can persist for large $m$. From here, it may be concluded that system (2) is always persistent for small subsidy input rate in the presence of nonlinear prey refuge function. Again, Figures 16 and 17, respectively, show that, in the absence of fear effect $(k=0)$, the equilibria $E_{3}$ and $E^{*}$ are approaching towards stable state by excluding the existence of oscillatory behaviour taking $m$ as the bifurcating parameter. In this manner, predator population also survives in ecosystem for large coefficient of prey refuge parameter $m$.

A "transcritical bifurcation" (BP) occurs at $d_{1}^{[\mathrm{TC}]}=5.5$ around $E_{1}$. At this point, exactly one eigenvalue of the Jacobian matrix is zero and others have negative real parts. Bifurcation diagram 32 depicts that when $d_{1}<d_{1}^{[\mathrm{TC}]}$, then $E_{1}$ 


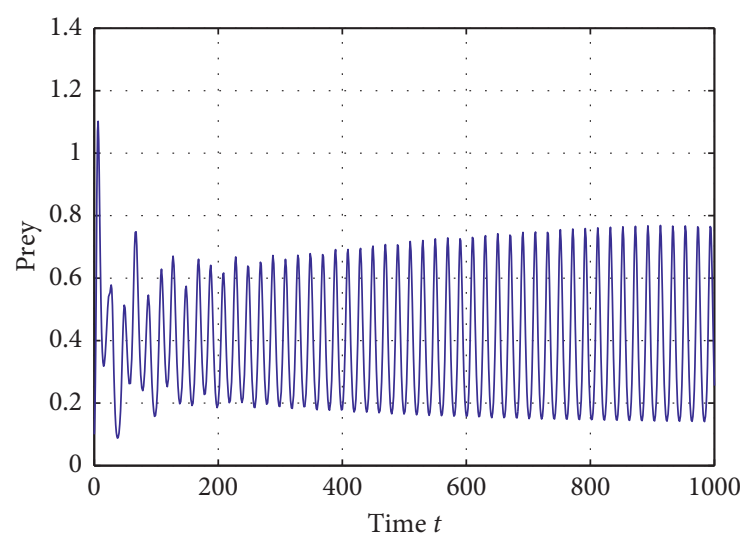

(a)

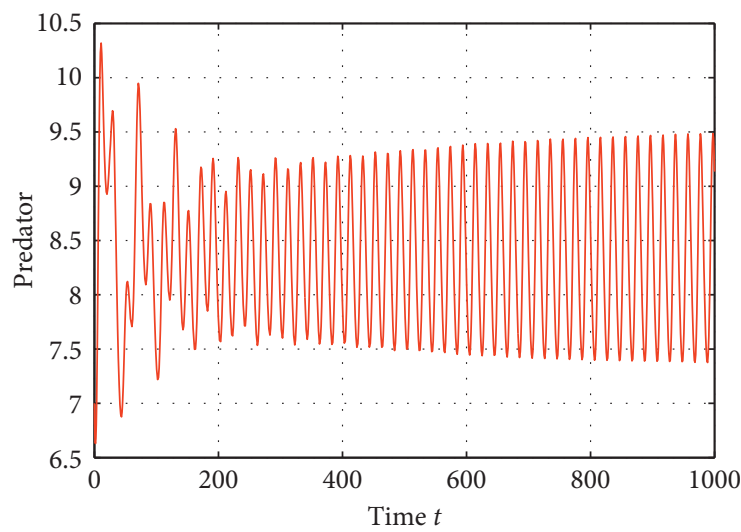

(c)

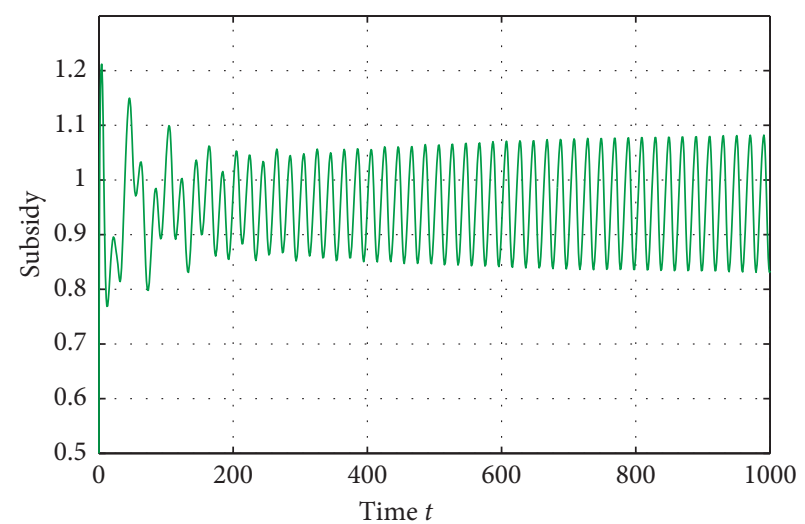

(b)

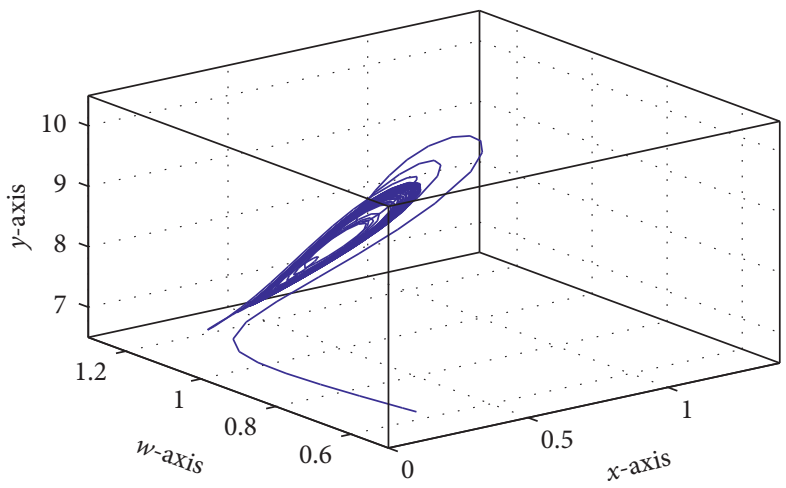

(d)

Figure 28: (a-c) oscillatory nature of prey, subsidy, and predator, respectively, with time and (d) stable limit cycle around $E^{*}(0.385717,0.950363,8.34509)$ when $\tau=25 \in\left(\tau_{1}^{+}=22.7845, \tau_{1}^{-}=37.7710\right)$ corresponding to the data set of Figure 9. (a) Oscillation of prey. (b). Oscillation of subsidy. (c) Oscillation of predator. (d) Phase portrait (closed orbit).

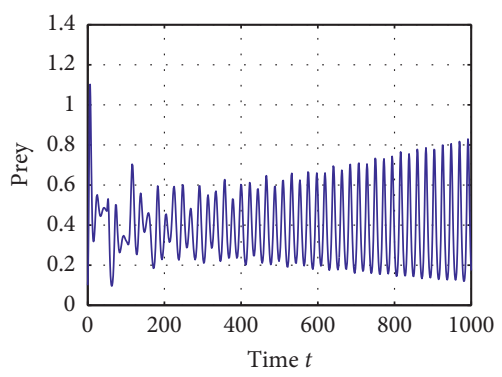

(a)

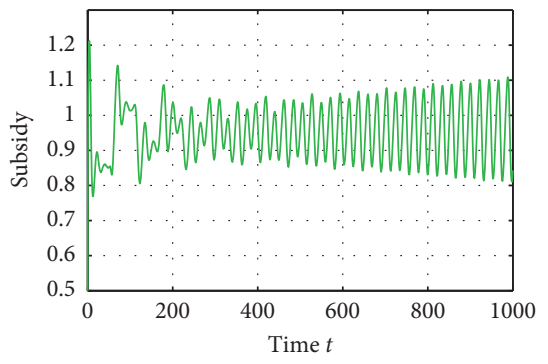

(b)

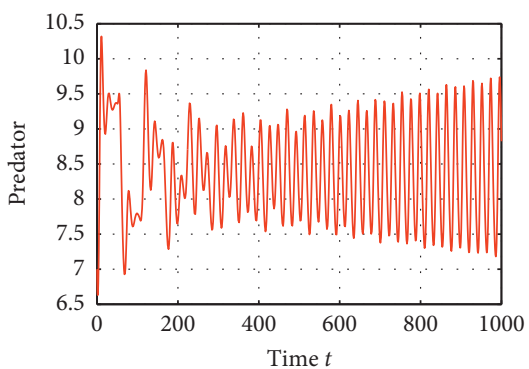

(c)

FIGURE 29: (a-c) Oscillatory nature of prey, subsidy, and predator, respectively, with time around $E^{*}(0.385717,0.950363,8.34509)$ when $\tau=50>\tau_{2}^{+}=41.5037$ corresponding to the data set of Figure 9. (a) Oscillation of prey. (b). Oscillation of subsidy. (c) Oscillation of predator.

is unstable, and when $d_{1}>d_{1}^{[\mathrm{TC}]}$, then $E_{1}$ is stable. Also, Figure 18 exhibits that when $d_{3}<d_{3}^{[\mathrm{TC}]}=0.1768$, then $E_{2}$ is unstable, and when $d_{3}>d_{3}^{[\mathrm{TC}]}=0.1768$, then $E_{2}$ is stable. So, a transcritical bifurcation is exhibited at $d_{3}^{[\mathrm{TC}]}=0.1768$ around $E_{2}$. Similarly, Figures 19 and 20 depict the transcritical bifurcation diagrams around equilibrium $E_{4}$ and $E_{5}$ taking $d_{1}\left(d_{1}^{[\mathrm{TC}]}=1.2238\right)$ and $d_{3}\left(d_{3}^{[\mathrm{TC}]}=0.61\right)$ as bifurcation parameter, respectively.
6.1. Effect of Time-Delay on Population Dynamics. Now, let us perform the numerical computations to validate the analytical results of the delayed model (60). For the parameter set $\left\{r=5.5, d_{1}=0.4, d_{2}=0.3, d_{3}=0.2, a_{1}=0.6\right.$, $a_{2}=0.98, \quad a_{3}=0.8, \quad b_{1}=2.5, \quad c_{1}=0.85, \quad c_{2}=0.7, \quad A=0$, $a=1.1, m=0.4\}$, equation (72) has exactly one positive root 0.2246 (correct up to four decimal places). So, from Theorem 18 , the planer equilibrium point $E_{3}(1.20053,0,6.49267)$ is 


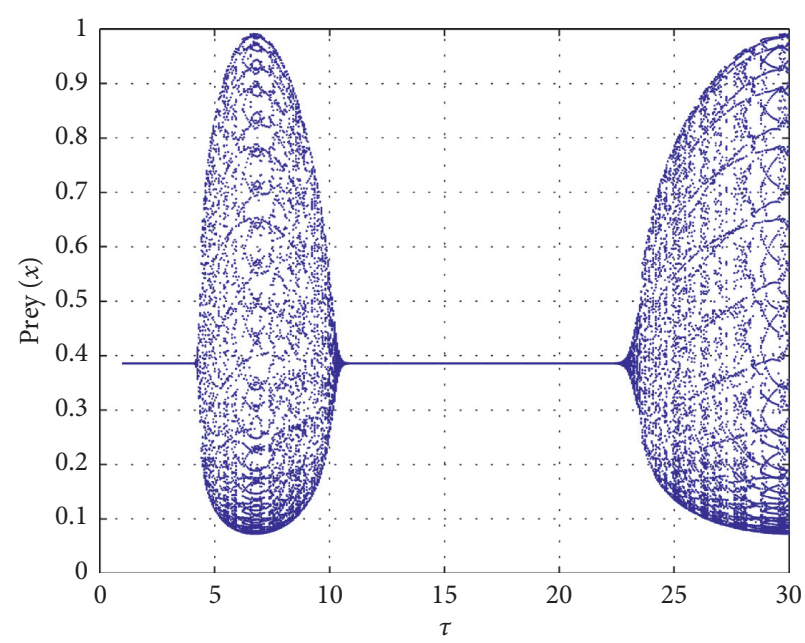

(a)

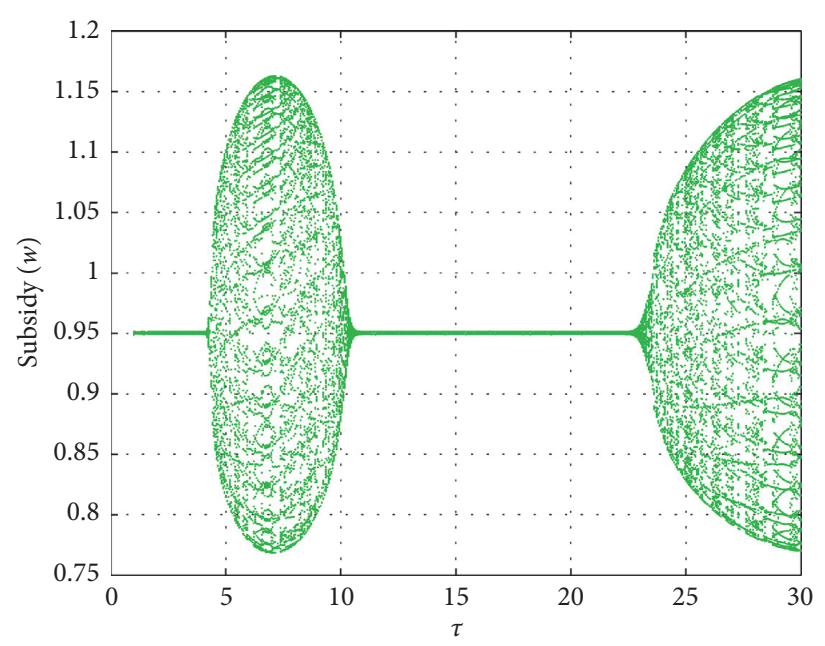

(b)

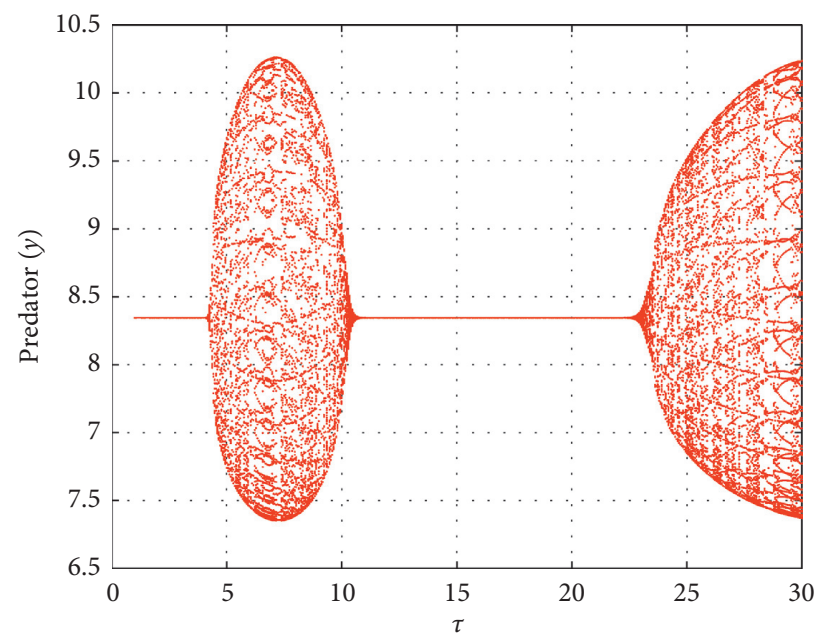

(c)

Figure 30: Bifurcation diagram around $E^{*}$ considering $\tau$ as independent variable and the remaining parameters are fixed as in dataset of Figure 9. Here, $\tau$ runs from 0 to 30. (a) Bifurcation diagram of $x$. (b) Bifurcation diagram of $w$. (c) Bifurcation diagram of $y$.

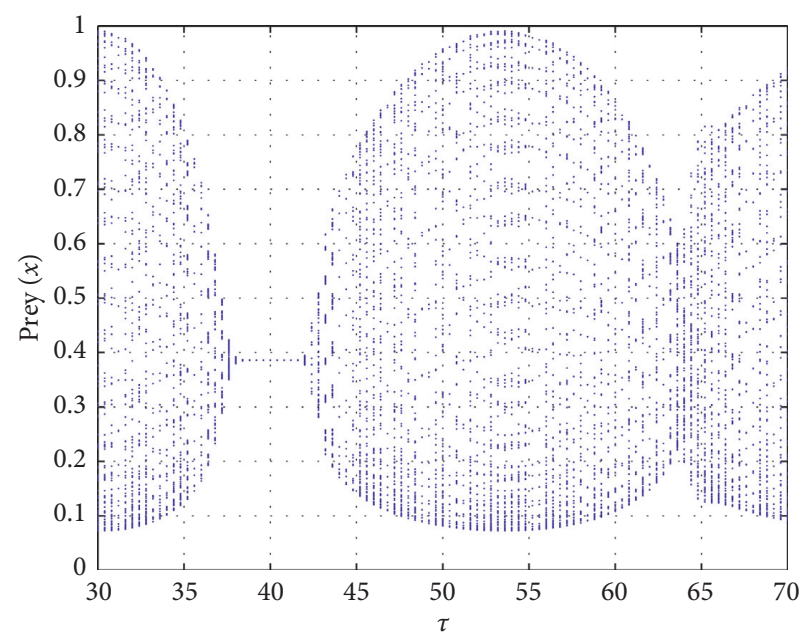

(a)

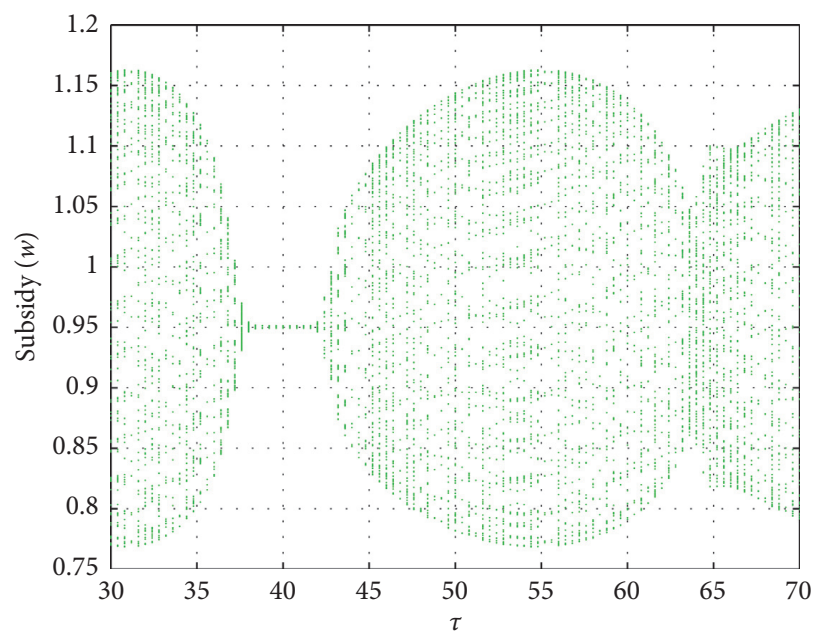

(b)

Figure 31: Continued. 


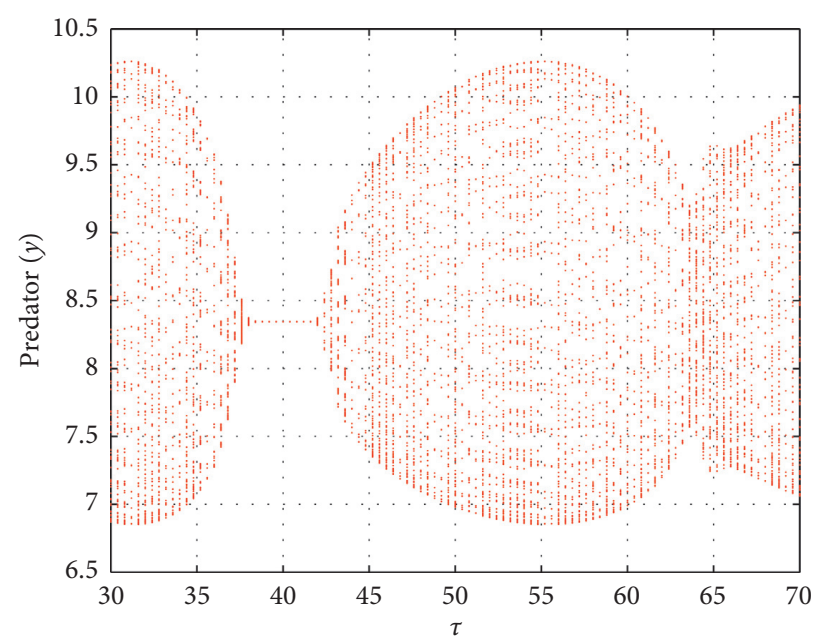

(c)

FiguRE 31: Bifurcation diagram around $E^{*}$ regarding $\tau$ as bifurcation parameter when $\tau$ runs from 30 to 70 and parameters are chosen from the data set of Figure 9. (a) Bifurcation diagram of $x$. (b) Bifurcation diagram of $w$. (c) Bifurcation diagram of $y$.

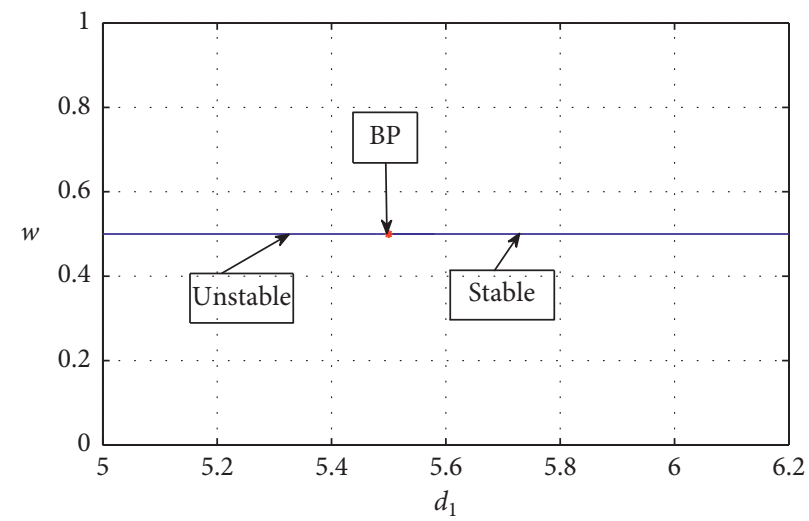

Figure 32: Transcritical bifurcation diagram around $E_{1}(0,0.5,0)$ considering $d_{1}$ as bifurcation parameter and others parameters are the same as in Figure 2. Here $d_{1}^{[\mathrm{TC}]}=5.5$.

stable when $\tau \in\left[0, \tau^{\prime *}=2.9272\right)$ and unstable when $\tau>\tau^{\prime *}=2.9272$. Stable time series and stable phase trajectory of $E_{3}(1.20053,0,6.49267)$ are shown in Figure 21 when $\tau=2<\tau^{\prime *}=2.9272$. Also, Figure 22 depicts the corresponding unstable behaviour of $E_{3}$ when $\tau=3.5>\tau^{\prime *}=2.9272$. Moreover, Figure 23 presents the supercritical Hopf bifurcation diagram around $E_{3}$ taking $\tau$ as bifurcation parameter.

For the parameter set $\left\{r=5.5, d_{1}=0.4, d_{2}=0.3\right.$, $d_{3}=0.2, a_{1}=0.6, a_{2}=0.98, a_{3}=0.8, b_{1}=2.5, c_{1}=0.85$, $\left.c_{2}=0.7, A=2, a=1.1, m=0.4\right\}$, equation (91) has exactly two positive roots, 0.1127 and 0.0526 (correct up to four decimal places). Then from Theorem 20, we have calculated $\tau_{0}^{+}=4.0652, \tau_{0}^{-}=10.3836, \tau_{1}^{+}=22.7845, \tau_{1}^{-}=37.7710$, and $\tau_{2}^{+}=41.5037$. The interior equilibrium $E^{*}(0.385717$, $0.950363,8.34509)$ is locally asymptotically stable when $\tau \in[0,4.0652),(10.3836,22.7845),(37.7710,41.5037)$ and unstable when $\tau \in(4.0652,10.3836),(22.7845,37.7710)$ and $\tau>$ 41.5037. At $\tau=\tau_{k}^{ \pm}, k=0,1$ and $\tau_{2}^{+}$, Hopf bifurcation appears around $E^{*}$. Figures $24-26$ depict the stable nature of $E^{*}$ for $\tau \in[0,4.0652),(10.3836,22.7845),(37.7710,41.50$ 37) respectively. Also, unstable behaviour of $E^{*}$ is presented in Figures 27-29 for $\tau \in(4.0652,10.3836),(22.7845,37.77$ 10) and $\tau>\tau_{2}^{+}=41.5037$, respectively. The corresponding bifurcation diagrams are depicted in Figures 30 and 31 .

\section{Conclusion}

We have analyzed a system for generalist predator which utilizes more than one food source: predator-prey-subsidy model of non-Kolmogorov form introducing nonlinear prey refuge function and the effect of fear felt by prey population. Our main interest is to find the situations such that dynamical stability and instability appear so as to make out more fully how subsidy may influence the predator and their prey. It has been shown that the solutions of system (2) remain positively invariant always and they are asymptotically uniformly bounded. These, in turn, imply that system (2) is biologically well-behaved. Existence 
criteria and stable behaviour of all the biologically meaningful equilibria have been discussed. It has to be noted that Hopf bifurcations are exhibited around $E_{3}$ (subsidy free) and $E^{*}$ (interior) of system (2) considering $k$ as a bifurcating parameter (see Figures 4-6, 9, 11, and 12). Also, observing Figures 6 and 12 , it can be concluded that high levels of fear can stabilize system (2) by excluding the existence of periodic solutions. These phenomena are biologically significant because prey species are aware after a certain level of fear; i.e., after a certain level of fear, they are not affected as they are aware and show signs of habituation.

Moreover, this work derives transcritical bifurcations (local bifurcation of codimension 1) at the various equilibrium points $E_{1}, E_{2}, E_{4}$, and $E_{5}$, respectively (see Figures 18-20 and 32).

Also, we have discussed numerically the influences of coefficient of prey refuge parameter $m$ on the nature of the equilibrium points $E_{3}$ (zero subsidy input rate) and $E^{*}$ (fixed small subsidy input rate) irrespective of fear level $k$. Noting Figures 14-17, it is observed that both the prey and predator species always persist in ecosystem due to continuous increment of coefficient of prey refuge. But Figures 10 and 13 depict that, irrespective of fear level, a highly subsidized predator should indeed drive the prey population towards extinction regardless of whether the prey and subsidy arise in the same habitat. This phenomenon is ecologically meaningful because the prey population cannot get enough time to protect themselves from predation risk for enormous growth of predator at high subsidy input rate. So, the prey population is leading towards extinction, but the predator species can easily survive in ecosystem with the help of resource subsidy. Thus, the study of system (2) is ecologically very significant.

In reality, fear effect does not instantaneously reduce the birth of a prey population, but some time lag should be needed to create an impact on the birth rate of the prey population. We have considered that there is a time-delay on the impact of fear to the birth rate of prey, from the instance it perceives the fear of predator through any means. So, the incorporation of time-delay makes system (60) more realistic. It is noted that delay parameter $\tau$ has a significant role because there exists a threshold value $\tau^{\prime *}$ such that stable behaviour of planer equilibrium point $E_{3}$ (in the absence of subsidy input rate) switches to oscillatory nature when $\tau$ passes through its threshold value $\tau^{\prime *}$; i.e., the vector fields for $\tau<\tau^{\prime *}$ and $\tau>\tau^{\prime *}$ are qualitatively different. So, system (60) exhibits a supercritical Hopf bifurcation around $E_{3}$ considering $\tau$ as bifurcation parameter (see Figures 21-23). Also, a rigorous study of the stability and bifurcation of interior equilibrium point $E^{*}$ has been performed. Our analysis describes that the delay within a certain specified range could maintain the stable behaviour of $E^{*}$. On the other hand, the delay could drive the system into an unstable state. Hence, the study of the timedelay parameter has a regulatory impact on the whole system.

\section{Data Availability}

The data used to support the findings of the study are available within the article.

\section{Conflicts of Interest}

The authors declare that they have no conflicts of interest.

\section{Acknowledgments}

The authors are grateful to Dr. Eberhard O. Voit (editor) for his careful reading, valuable comments, and helpful suggestions, which have helped them to improve the presentation of this work significantly. This research was completed during the visit of Prof. G.P. Samanta to the Institute of Mathematics of the University of Santiago de Compostela. This work has been partially supported by the Agencia Estatal de Investigacion (AEI) of Spain, cofinanced by the European Fund for Regional Development (FEDER) corresponding to the 2014-2020 multiyear financial framework project MTM2016-75140-P and by Xunta de Galicia under grant ED431C 2019/02.

\section{References}

[1] S. Creel and D. Christianson, "Relationships between direct predation and risk effects," Trends in Ecology \& Evolution, vol. 23, no. 4, pp. 194-201, 2008.

[2] W. Cresswell, "Predation in bird populations," Journal of Ornithology, vol. 152, no. S1, pp. 251-263, 2010.

[3] S. Creel, D. Christianson, S. Liley, and J. A. Winnie, "Predation risk affects reproductive physiology and demography of elk," Science, vol. 315, no. 5814, p. 960, 2007.

[4] L. Y. Zanette, A. F. White, M. C. Allen, and M. Clinchy, "Perceived predation risk reduces the number of offspring songbirds produce per year," Science, vol. 334, no. 6061, pp. 1398-1401, 2011.

[5] X. Wang, L. Zanette, and X. Zou, "Modelling the fear effect in predator-prey interactions," Journal of Mathematical Biology, vol. 73, no. 5, pp. 1179-1204, 2016.

[6] A. Das and G. P. Samanta, "Modeling the fear effect on a stochastic prey-predator system with additional food for the predator," Journal of Physics A: Mathematical and Theoretical, vol. 51, Article ID 465601, 2018.

[7] A. Das and G. P. Samanta, "Modelling the fear effect in a twospecies predator-prey system under the influence of toxic substances," Rendiconti del Circolo Matematico di Palermo Series 2, vol. 70, 2021.

[8] M. Das and G. P. Samanta, "A prey-predator fractional order model with fear effect and group defense," International Journal of Dynamics and Control, vol. 9, no. 1, pp. 334-349, 2020.

[9] M. Das and G. P. Samanta, "A delayed fractional order food chain model with fear effect and prey refuge," Mathematics and Computers in Simulation, vol. 178, pp. 218-245, 2021.

[10] S. Mondal, A. Maiti, and G. P. Samanta, "Effects of fear and additional food in a delayed predator-prey model," Biophysical Reviews and Letters, vol. 13, no. 04, pp. 157-177, 2018.

[11] S. Mondal and G. P. Samanta, "Dynamics of a delayed predator-prey interaction incorporating nonlinear prey refuge under the influence of fear effect and additional food," Journal of Physics A: Mathematical and Theoretical, vol. 53, no. 29, Article ID 295601, 2020.

[12] S. Mondal and G. P. Samanta, "Time-delayed predator-prey interaction with the benefit of antipredation response in presence of refuge," Zeitschrift fur Naturforschung A, vol. 76, no. 1, pp. 23-42, 2021. 
[13] S. Mondal and G. P. Samanta, "Impact of fear on a predatorprey system with prey-dependent search rate in deterministic and stochastic environment," Nonlinear Dynamics, vol. 104, pp. 2931-2959, 2021.

[14] D. Sahoo and G. P. Samanta, "Comparison between two tritrophic food chain models with multiple delays and antipredation effect," International Journal of Biomathematics, vol. 14, no. 3, Article ID 2150008, 2021.

[15] D. Sahoo and G. P. Samanta, "Impact of fear effect in a two prey-one predator system with switching behaviour in predation," Differential Equations and Dynamical Systems, vol. 29, 2021.

[16] X. Wang and X. Zou, "Modeling the fear effect in predatorprey interactions with adaptive avoidance of predators," Bulletin of Mathematical Biology, vol. 79, pp. 1325-1359, 2017.

[17] Y. Wang and X. Zou, "On a predator-prey system with digestion delay and anti-predation strategy," Journal of Nonlinear Science, vol. 30, pp. 1432-1467, 2020.

[18] S. Samaddar, M. Dhar, and P. Bhattacharya, "Effect of fear on prey-predator dynamics: Exploring the role of prey refuge and additional food," Chaos: An Interdisciplinary Journal of Nonlinear Science, vol. 30, no. 6, Article ID 063129, 2020.

[19] H. Zhang, Y. Cai, S. Fu, and W. Wang, "Impact of the fear effect in a prey-predator model incorporating a prey refuge," Applied Mathematics and Computation, vol. 356, pp. 328-337, 2019.

[20] J. Ghosh, B. Sahoo, and S. Poria, "Prey-predator dynamics with prey refuge providing additional food to predator," Chaos, Solitons \& Fractals, vol. 96, pp. 110-119, 2017.

[21] E. González-Olivares, B. González-Yañez, R. Becerra-Klix, and R. Ramos-Jiliberto, "Multiple stable states in a model based on predator-induced defenses," Ecological Complexity, vol. 32, pp. 111-120, 2017.

[22] T. K. Kar, "Stability analysis of a prey-predator model incorporating a prey refuge," Communications in Nonlinear Science and Numerical Simulation, vol. 10, no. 6, pp. 681-691, 2005.

[23] S. Mondal and G. P. Samanta, "Dynamics of an additional food provided predator-prey system with prey refuge dependent on both species and constant harvest in predator," Physica A: Statistical Mechanics and Its Applications, vol. 534, Article ID 122301, 2019.

[24] C. T. Darimont, P. C. Paquet, and T. E. Reimchen, "Spawning salmon disrupt trophic coupling between wolves and ungulate prey in coastal British Columbia," BMC Ecol, vol. 8, pp. 1-12, 2008.

[25] J. Halaj and D. H. Wise, "Impact of a detrital subsidy on trophic cascades in a terrestrial grazing food web," Ecology, vol. 83, no. 11, pp. 3141-3151, 2002.

[26] J.-A. Henden, R. A. Ims, N. G. Yoccoz, P. Hellstorm, and A. Angerbjorn, "Strength of asymmetric competition between predators in food webs ruled by fluctuating prey: The case of foxes in tundra," Oikos, vol. 119, no. 1, pp. 27-34, 2010.

[27] R. A. Garrott, J. E. Bruggeman, M. S. Becker, S. T. Kalinowski, and P. J. White, "Evaluating prey switching in wolf-ungulate systems," Ecological Applications, vol. 17, no. 6, pp. 1588-1597, 2007.

[28] A. L. Nevai and R. A. Van Gorder, "Effect of resource subsidies on predator-prey population dynamics: a mathematical model," Journal of Biological Dynamics, vol. 6, no. 2, pp. 891-922, 2012.

[29] A. Erbach, F. Lutscher, and G. Seo, "Bistability and limit cycles in generalist predator-prey dynamics," Ecological Complexity, vol. 14, pp. 48-55, 2013.
[30] A. Morozov and S. Petrovskii, "Feeding on multiple sources: towards a universal parameterization of the functional response of a generalist predator allowing for switching," PLoS One, vol. 8, no. 9, Article ID e74586, 2013.

[31] P. Sunde, K. Thorup, L. B. Jacobsen, and C. Rahbek, "Weather conditions drive dynamic habitat selection in a generalist predator," PLoS ONE, vol. 9, no. 2, Article ID e88221, 2014.

[32] E. L. Clare, E. E. Fraser, H. E. Braid, M. B. Fenton, and P. D. N. Hebert, "Species on the menu of a generalist predator, the eastern red bat (Lasiurus borealis): using a molecular approach to detect arthropod prey," Molecular Ecology, vol. 18, no. 11, pp. 2532-2542, 2009.

[33] F. Díaz-Ruiz, M. Delibes-Mateos, J. L. García-Moreno, J. María López-Martín, C. Ferreira, and P. Ferreras, "Biogeographical patterns in the diet of an opportunistic predator: the red foxVulpes vulpesin the Iberian Peninsula," Mammal Review, vol. 43, no. 1, pp. 59-70, 2013.

[34] J. D. Willson and W. A. Hopkins, "Prey morphology constrains the feeding ecology of an aquatic generalist predator," Ecology, vol. 92, no. 3, pp. 744-754, 2011.

[35] A. Das and G. P. Samanta, "Modelling the effect of resource subsidy on a two-species predator-prey system under the influence of environmental noises," International Journal of Dynamics and Control, vol. 9, 2021.

[36] Y. Xu, A. L. Krause, and R. A. Van Gorder, "Generalist predator dynamics under Kolmogorov versus non-Kolmogorov models," Journal of Theoretical Biology, vol. 486, Article ID 110060, 2020.

[37] L. G. Adams, S. D. Farley, C. A. Stricker et al., "Are inland wolf-ungulate systems influenced by marine subsidies of pacific salmon?" Ecological Applications, vol. 20, no. 1, pp. 251-262, 2010.

[38] C. S. Holling, "The components of predation as revealed by a study of small-mammal predation of the european pine sawfly," The Canadian Entomologist, vol. 91, no. 5, pp. 293-320, 1959.

[39] C. S. Holling, "Some characteristics of simple types of predation and parasitism," The Canadian Entomologist, vol. 91, no. 7, pp. 385-398, 1959.

[40] C. S. Holling, "The functional response of predators to prey density and its role in mimicry and population regulation," Memoirs of the Entomological Society of Canada, vol. 97, no. S45, pp. 5-60, 1965.

[41] K. Sarkar, S. Khajanchi, P. C. Mali, and J. J. Nieto, "Rich dynamics of a predator-prey system with different kinds of functional responses," Complexity, vol. 2020, Article ID 4285294, 2020.

[42] J. K. Hale, Theory of Functional Differential Equations, Springer-Verlag, New York, NY, USA, 1977.

[43] L. Perko, Differential Equations and Dynamical Systems, Springer, Berlin, Germany, 2001.

[44] J. D. Murray, Mathematical Biology, Springer, Berlin, Germany, 1993.

[45] S. Khajanchi and J. J. Nieto, "Mathematical modeling of tumor-immune competitive system, considering the role of time delay," Applied Mathematics and Computation, vol. 340, pp. 180-205, 2019.

[46] Y. Kuang, Delay Differential Equation with Application in Population Dynamics, Academic Press, New York, NY, USA, 1993.

[47] S. Mondal and G. P. Samanta, "Dynamical behaviour of a twoprey and one-predator system with help and time delay," Energy, Ecology and Environment, vol. 5, no. 1, pp. 12-33, 2020. 
[48] S. Mondal and G. P. Samanta, "Pelican-Tilapia interaction in Salton sea: an eco-epidemiological model with strong Allee effect and additional food," Modeling Earth Systems and Environment, vol. 7, 2021.

[49] D. Sahoo, S. Mondal, and G. P. Samanta, "Interaction among toxic phytoplankton with viral infection and zooplankton in presence of multiple time delays," International Journal of Dynamics and Control, vol. 9, no. 1, pp. 308-333, 2021.

[50] S. Ruan and J. Wei, "On the zeros of transcendental functions with applications to stability of delay differential equations with two delays," Dynamics of Continuous, Discrete and Impulsive Systems Series B: Applications and Algorithms, vol. 10, pp. 863-874, 2003.

[51] H. Freedman and V. Rao, "The trade-off between mutual interference and time lags in predator-prey systems," Bulletin of Mathematical Biology, vol. 45, no. 6, pp. 991-1004, 1983. 